Draft: 9/15/06

\title{
Survey of \\ Patient Mobility and Lift Technologies Toward Advancements and Standards
}

Roger Bostelman, James Albus

July 2006

NISTIR 7384

Disclaimer: NIST does not endorse products discussed within this report nor manufacturers of these products. Products mentioned are for information purposes only and are not expressed as an endorsement for them or their manufacturer. 


\section{EXECUTIVE SUMMARY}

This survey provides a review of example off-the-shelf lift and wheelchair products on the market today. Also, it provides a review of example research in these areas including advancements in intelligent wheelchair designs and capabilities. We have focused on lift devices, wheelchairs, the combination of wheelchairs and lift devices, rehabilitation and standards. What appears to be lacking, as determined by our research for this survey, are the following points regarding wheelchair dependents:

- There is limited research and development in the area of advanced patient lift devices, beyond standers, that are combined with wheelchairs to allow lift above approximately $5.5 \mathrm{~cm}$ (14 in) to reach upper shelves and even upper floors,

- There is limited research and development in the area of patient lift devices combined with wheelchairs to place patients on toilets, chairs, or beds or in bathtubs,

- There has been and is research and development ongoing in the area of advanced intelligent wheelchairs but, has yet to reach the commercial market due to: cost, lacking robust capabilities, little or no clinical trials, or other issues,

- There is advanced research ongoing to study navigation with 2 dimensional sensors such as LADAR (laser detection and ranging) but, minimal efforts toward 3D environment sensors use on wheelchairs beyond cameras is being studied,

- There are only limited wheelchair exercise equipment devices available and none are attached to the wheelchair to provide partial weight bearing by patients toward leg rehabilitation,

- $\quad$ There are limited patient lift devices on the market and none have intelligent capability to navigate in medical facilities on their own to "come when called" as needed by limited nursing staffs,

- There are no standards for intelligent wheelchairs and no standards that are guiding a concerted effort toward optimal intelligent wheelchair designs.

Based on the above points, NIST recommends that the following research and development continue or begin:

- Develop higher and more capable lift wheelchairs allowing WCD's to reach above currently achievable heights to be more independent at home and in the work place

- Develop wheelchairs that can pick up and place WCD's on toilets, chairs, beds and in bathtubs,

- Develop robust and affordable intelligent wheelchairs that are championed by medical funding agencies towards commercial products,

- Develop advanced wheelchairs that use 3D environment sensors for robust world sensing and modeling to plan appropriate and safe robot paths,

- Develop advanced, partial to full weight bearing, rehabilitation devices that are integrated into wheelchairs for easy user accessibility and use by the wheelchair dependent,

- Develop patient lift devices that assist nursing staff through advanced mobility and lift capabilities to act as a nursing assistant when there is limited nursing staff,

- Develop standards for intelligent wheelchairs that drive robust designs for the optimal mobility device for WCD’s.

Also, the following points should be addressed regarding funding for this R\&D: 
Draft: 9/15/06

- Develop US Government programs to provide standards, performance measurements and advanced technology development needed to reduce the healthcare assistive device costs to the public,

- Provide comparable investment in this area of technology to European investments. 


\section{Table of Contents}

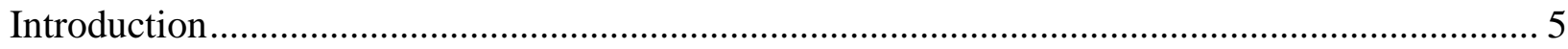

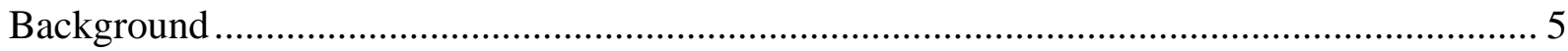

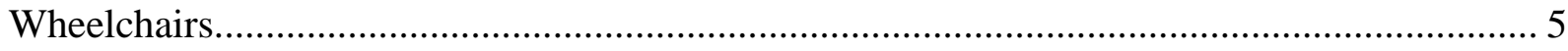

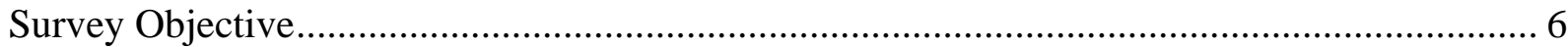

Why Advance Wheelchair Lift Mechanisms? ................................................................. 7

NIST Healthcare Mobility Project ................................................................................... 7

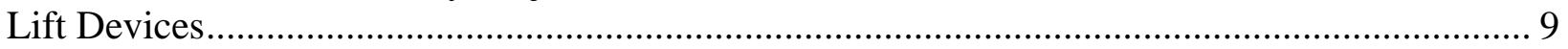

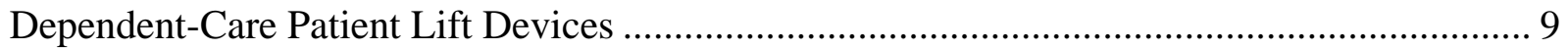

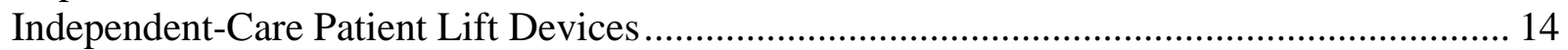

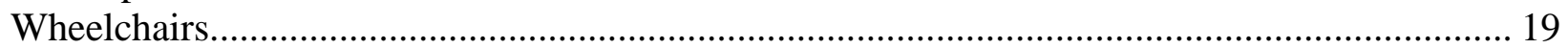

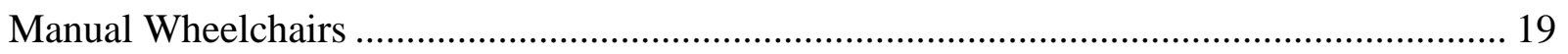

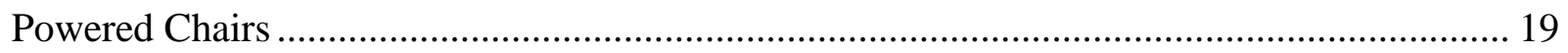

Power-Assist Manual Wheelchairs ......................................................................... 21

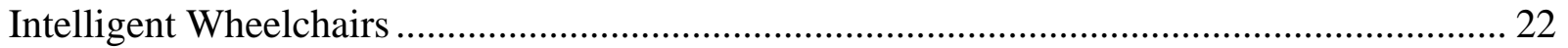

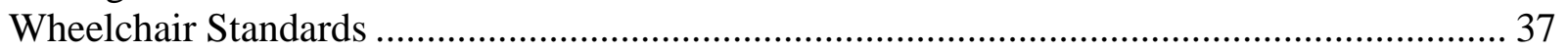

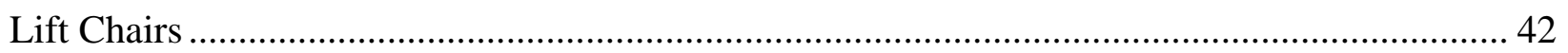

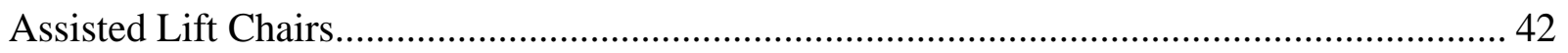

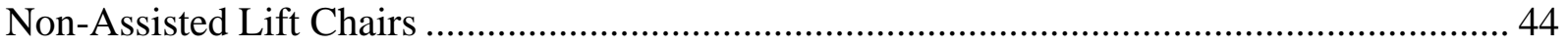

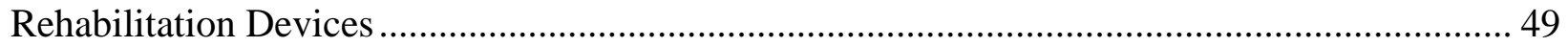

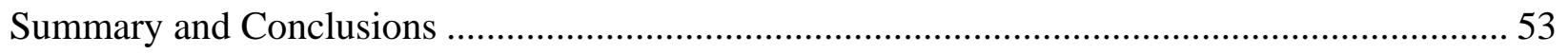

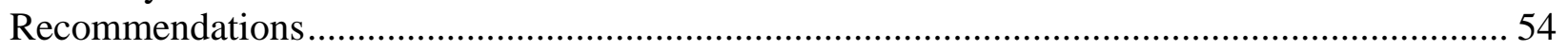

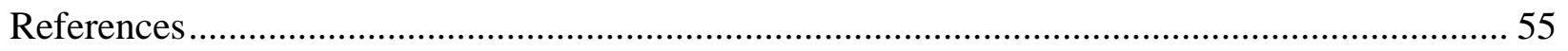

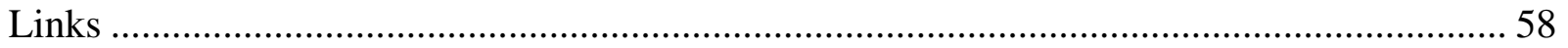

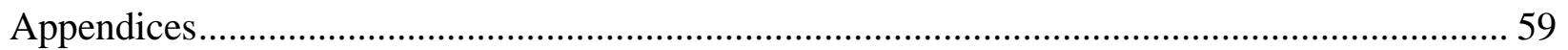

Appendix 1 - Lift Device Research Summary .................................................................. 59

Appendix 2 - Patent Search - "Patient Lift” AND "Wheelchair”......................................... 66

Appendix 3 - Lift Devices List ................................................................................... 73

Appendix 4 - Patient Characteristics List .................................................................. 77

Appendix 5 - Safe Patient Handling and Movement Algorithms .......................................... 81 


\section{INTRODUCTION}

\section{Background}

[Pollack, 2005] says “today, approximately 10 percent of the world's population is over 60; by 2050 this proportion will have more than doubled" and "the greatest rate of increase is amongst the oldest old, people aged 85 and older." She follows by adding that this group is therefore subject to both physical and cognitive impairments more than younger people. These facts have a profound impact on how the world will keep the elderly independent as long as possible from caregivers. Both physical and cognitive diminishing abilities address the body and the mental process of knowing, including aspects such as awareness, perception, reasoning, intuition and judgment. [Dictionary.com] Assistive technology for the mobility impaired includes the wheelchair, lift aids and other devices, all of which have been around for centuries. However, the patient typically or eventually requires assistance to use the device; whether to push the wheelchair, to lift themselves from the bed to a chair or to the toilet or for guiding the patient through cluttered areas. With fewer caregivers and more elderly in the near future, there is a need for improving these devices to provide them independent assistance. As further background, the authors have included sections on wheelchairs and lift devices.

\section{Wheelchairs}

Wheelchairs have been around for four hundred years since the first dedicated wheelchair, called an "invalids chair," was invented for Phillip II of Spain. Later, in 1932, engineer, Harry Jennings, built the first folding, tubular steel wheelchair similar to what is in use today. That chair was built for a paraplegic friend of Jennings called Herbert Everest. Together they founded Everest \& Jennings. [Bellis]

There has been an increasing need for wheelchairs over time. In [van der Woude , 1999] they state: "Mobility is fundamental to health, social integration and individual well-being of the human being. Henceforth, mobility must be viewed as being essential to the outcome of the rehabilitation process of wheelchair dependent persons and to the successful (re-)integration into society and to a productive and active life. Many lower limb disabled subjects depend upon a wheelchair for their mobility. Estimated numbers for the Netherlands, Europe and USA are respectively 80,000, 2.5 million and 1.25 million wheelchair dependent individuals. Groups large enough to allow a special research focus and conference activity. Both the quality of the wheelchair, the individual work capacity, the functionality of the wheelchair/user combination, and the effectiveness of the rehabilitation program do indeed determine the freedom of mobility. Their optimization is highly dependent upon a continuous and high quality research effort, in combination with regular discussion and dissemination with practitioners. ...”

Just as important as wheelchairs are the lift devices and people who lift patients into wheelchairs and other seats, beds, automobiles, etc. The need for patient lift devices will also increase as generations get older. When considering if there is a need for patient lift devices, several references state the positive, for example:

- $\quad$ "The question is, what does it cost not to buy this equipment? A back injury can cost as much as $\$ 50,000$, and that's not even including all the indirect costs. If a nursing home can 
buy these lifting devices for $\$ 1,000$ to $\$ 2,000$, and eliminate a back injury that costs tens of thousands of dollars, that's a good deal,” [Marras]

- 1 in every 3 nurses become injured from the physical exertion put forth while moving nonambulatory patients; costing their employers $\$ 35,000$ per injured nurse. [Blevins]

- 1 in 2 non-ambulatory patients fall to the floor and become injured when being transferred from a bed to a wheelchair. - [U.S. Bureau of Labor Statistics, 1994]

- $\quad$ "Nursing and personal care facilities are a growing industry where hazards are known and effective controls are available," said OSHA Administrator John Henshaw. "The industry also ranks among the highest in terms of injuries and illnesses, with rates about $21 / 2$ times that of all other general industries..." - [OSHA Website]

- “Already today there are over 400,000 unfilled nursing positions causing healthcare providers across the country to close wings or risk negative outcomes. Over the coming years, the declining ratio of working age adults to elderly will further exacerbate the shortage. In 1950 there were 8 adults available to support each elder 65+, today the ratio is 5:1 and by 2020 the ratio will drop to 3 working age adults per elder person.” [Wasatch Digital IQ, 2003]

\section{Survey Objective}

For this survey, we set out to understand the state of technology for mobility devices for wheelchair dependents (WCD), including the standards that back them. As described in the Background section above, there is a need for smart wheelchairs as people are living longer than before, will typically become less mobile with time, and will have reduced cognitive abilities and yet will need and perhaps want to remain independent. With fewer, younger and more capable assistants available for these elders, it creates a need for personal robotic care. Standards are being set for these mobile devices including manual and powered devices. Intelligent powered chairs have not yet been standardized.

Moreover, toward full independence for WCD's and for elders, there is a need for patient lift devices to move them from one floor to the next, from the bed or chair to the toilet, to a wheelchair, to cars, and other places, etc. This study attempts to provide a state of the technology for these devices also. There exist safety standards for these devices but, is not a focus of this paper. In an effort to address these standards, Appendix 1 includes a listing and brief explanation of some safety standards, statistics, other references and manufacturers of patient lift devices.

A combination lift device and wheelchair has become available within the past several years. Some of these technologies will also be shown in following sections. They provide stair/curb climbing, lift to reach tall shelves, etc. Advances in this area are a primary focus for this survey. Standards for lift wheelchairs have not yet become available. 


\section{Why Advance Wheelchair Lift Mechanisms?}

Authors prediction:

- Wheelchair dependents want to be self-sufficient, even in a typical home

- WCD's and/or homeowners don't want the home changed due to costs and intrusive changes, or even radically exchanging homes (e.g., selling 2 story to buy a 1 story).

- Want to be mobile; pick from and place things on shelves and cabinets; be at eye level to others; sit in their favorite chair; use a standard toilet; perform household tasks (cook, clean, hobbies); etc.

- Want more than minimal devices (e.g., more than a wheelchair and trapeze)

- Would probably like to have even better features than non-WCD’s (e.g., 2 story lift) Also,

- Wheelchairs/powered chairs mobilize but, typically cannot lift (except 10” - 13” lift units) nor place WCD's in favorite chairs, etc. And assist devices are difficult to use.

- Gyro-stabilized lift chair cannot lift patient to reach upper cabinet shelf heights, it balances on two wheels (e.g., market acceptability?), and is relatively expensive as compared to powered chairs.

- Ceiling, patient, and other lifts do not have full user-controlled mobility and/or are usually location specific

- Need multiple devices for mobility and lift equating to more patient/insurance costs, and providing cumbersome maneuverability for the patient.

- Rehabilitation assistance is virtually non-existent with current wheelchairs, some basics in stander, walker, rollator technologies.

The National Institute of Child Health and Human Development (NICHD) released a Small Business Innovative Research Request for Assistance for Innovations in Powered Mobility Devices in 2003 [NCMRR, 2003] to reduce the weight and required power and improve the portability of these devices. No similar request has been sent to the public for lift wheelchair devices toward better independence of WCD's.

\section{NIST Healthcare Mobility Project}

NIST has been researching intelligent mobility systems for over 20 years through the Intelligent Systems Division's, Intelligent Control of Mobility Systems (ICMS) Program. These systems include projects mainly military, transportation and industrial devices. ICMS projects have greatly contributed to the advancement of mobile robots and can provide sound basis for healthcare mobile robots as well. An additional project at NIST is the Healthcare Mobility Project begun in 2004. Questions and answers regarding this project asked by NIST to better understand NIST involvement in this research are as follows:

How is it solved today and by whom?

Devices for wheelchair dependents and elderly are:

- Need specific

- Attempt to be very inexpensive

- Are mostly available in care centers, hospitals

- Typically require additional caregiver dependence. 
Who else is working on these goals/objectives?

- Universities performing intelligent wheelchair research

- Many companies exist to provide "need specific” devices

- Few exist, if any, to provide generic or multi-purpose tools, for example: wheelchairs that lift AND navigate on their own AND sense crosswalks AND sense negative obstacles (stairs) well before approaching them.

What is the new technical idea? Why can the developer succeed?

- Sensor technology has advanced well beyond the still-used ultrasonic sensors.

o CSEM SwissRanger 2, Sick LMS, Canesta D200, PmdTech 3-dimensional imagers

- Computer systems are much faster, smaller, less-expensive than ever before

- Powered chairs are off-the-shelf items now, several companies sell them

- Intelligence is just beginning to be built into powered chairs - e.g., doorway guidance

Why is the time right now?

- Standards exist for powered chairs, none exist for intelligent chairs that use advanced sensors, computers and actuation systems.

- Before intelligent chairs are commercialized and sold to the general public, a target safety design standard should be in place.

What is the impact if successful?

- Improved device capabilities

o Safety: e.g., Powered chairs that don't require stair blocks or caregiver watch; guidance for the blind

o Obstacle detection: e.g., know when operator is about to run into an obstacle; guidance for the blind

o Reduced dependency on caregivers for the elderly and disabled

Who are customers/stakeholders that will benefit?

- Elderly

- Wheelchair dependents

- Disabled persons

- Blind persons

- Nurses, Caregivers

- Impact to nurses (medical facilities) and caregivers by reducing the current $50 \%$ back injury rate

- Medical facilities and the public would benefit from lower insurance claims, fewer worker (nurses) days off

o Current shortage of nurses and caregivers

o Elderly population is expected to rise significantly around the world, especially in Japan.

How will you measure progress? 
- By setting and achieving the following project goals:

\section{Project Goals:}

To first understand what has been done in the area of wheelchair and lift devices for wheelchair dependents (WCD's) toward improved capabilities for these devices that would require less care-giver support. Second, to provide test methods and performance metrics, sensor data, standards and specifications necessary for intelligent assistive devices for wheelchair dependents. And third, if necessary, to provide an advanced general purpose mobility assistive device for WCD's that reduces potential injury to the WCD and/or care provider and while improving the quality of life for the WCD beyond what currently-available technology can provide.

The NIST Healthcare Mobility Project goal also provides carryover to other industries, where onboard intelligent vehicle control with lift capability is needed. Industries that could be considered for this type of lift and mobility are in manufacturing, distribution, and others where handicapped personnel need assistance lifting and maneuvering loads on a small scale. Here we provide an expansion of the Healthcare Mobility Project goal as follows:

\section{Other Industries Goal:}

To provide a general purpose mobility assistive device, including: relatively inexpensive, powered, safety, lift features, for maneuvering heavy (=/> than a person or two can lift) loads from floor-level or below to above-worker-level reach or beyond requiring abovethe-load-lift and mobility while reducing potential injury to workers and improving capabilities beyond what currently-available technology can provide.

\section{LIFT DEVICES}

Types of lift devices shown in this section are divided into dependent- and independent-care lift devices, including a separate section on powered lift devices.

\section{Dependent-Care Patient Lift Devices}

These devices allow patient lift (left) in a seated position or (middle and right) in a laying position. Both devices manually lift by pumping a hydraulic piston.
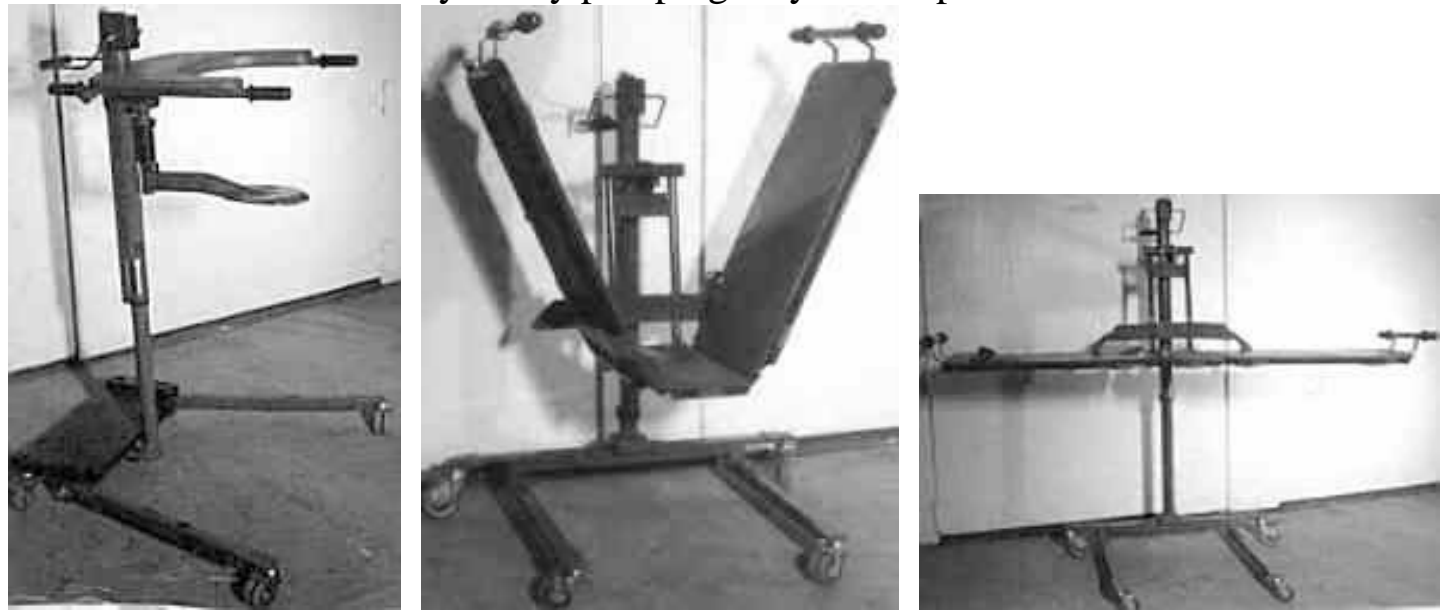
The Patient Pivot allows a person to be lifted from a seated position, once strapped to the Pivot, and rotated forward to allow patient placement on another seat. The rotate mechanism uses levers allowing the caregiver to supply reduced force relative to lifting without the device.

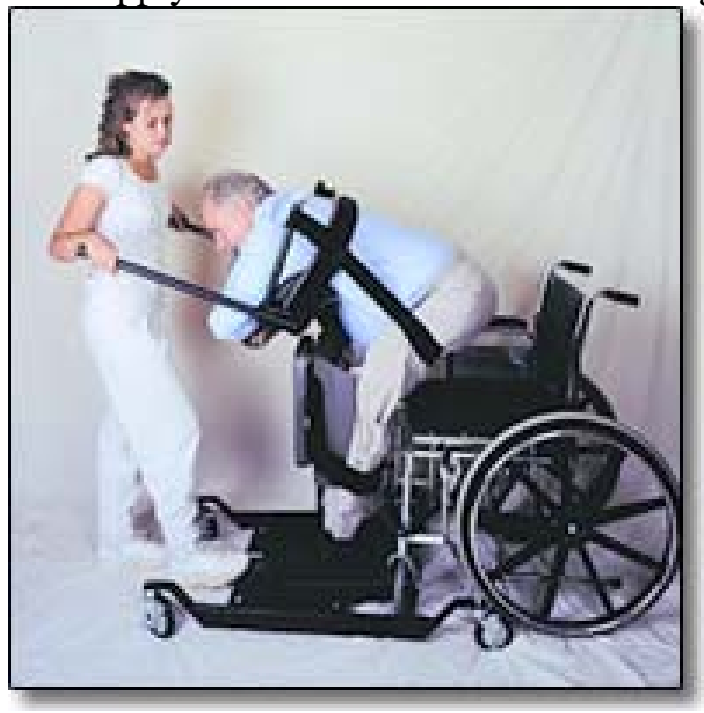

Once a hammock sheet is placed beneath the patient, lift devices such as this can be used to power lift the patient from a seated or laying position. When placed in a seat or bed, the sheet initially remains beneath the patient until removed. The device allows compliance for rotating the patient about the lift point and is manually mobilized by the caregiver.
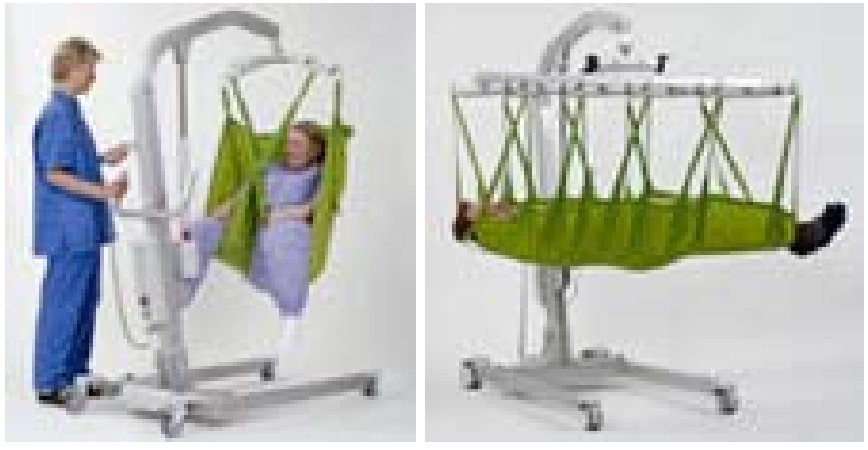

This newer version of the one shown above provides similar lift capability and also allows the legs to be power separated for easy patient access to the device and/or to accommodate access to wide-base seats. 


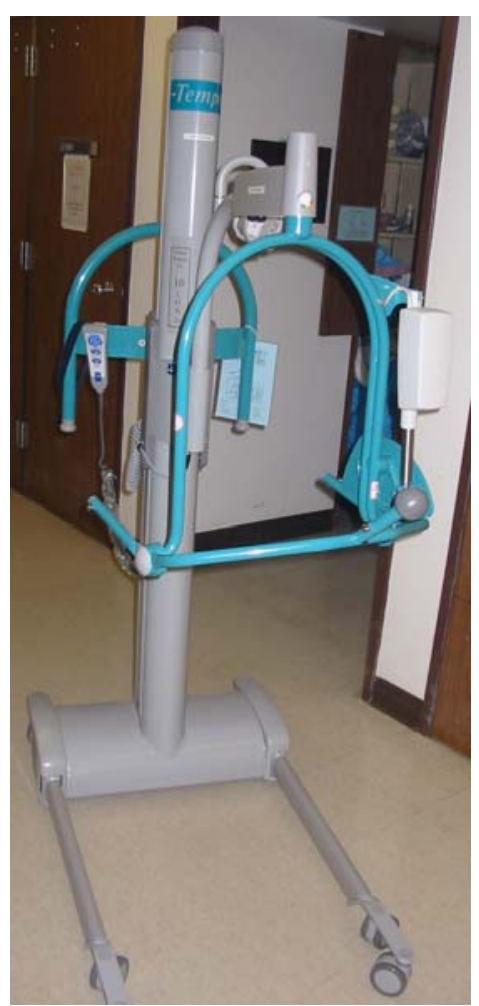

(thanks to UCSF - University of California San Francisco)

This similar device (left) as above allows patient lift from a seated position and uses a minimized harness to support the patient during lift. The smaller inset photo shown with the left photo is a sit to stand type device that uses a strap to pull from behind the patient moving him/her forward and a shin support in the front limiting forward motion. The single piston is positioned on the device to provide lift and minimize forward motion or pressure on the shins. The patient is then in a standing position with buttocks exposed to be placed in a seat. The back strap is then removed.

The device on the right is similar to the one shown in the inset photo where the patient is strapped from behind and is power-lifted to a standing position. 

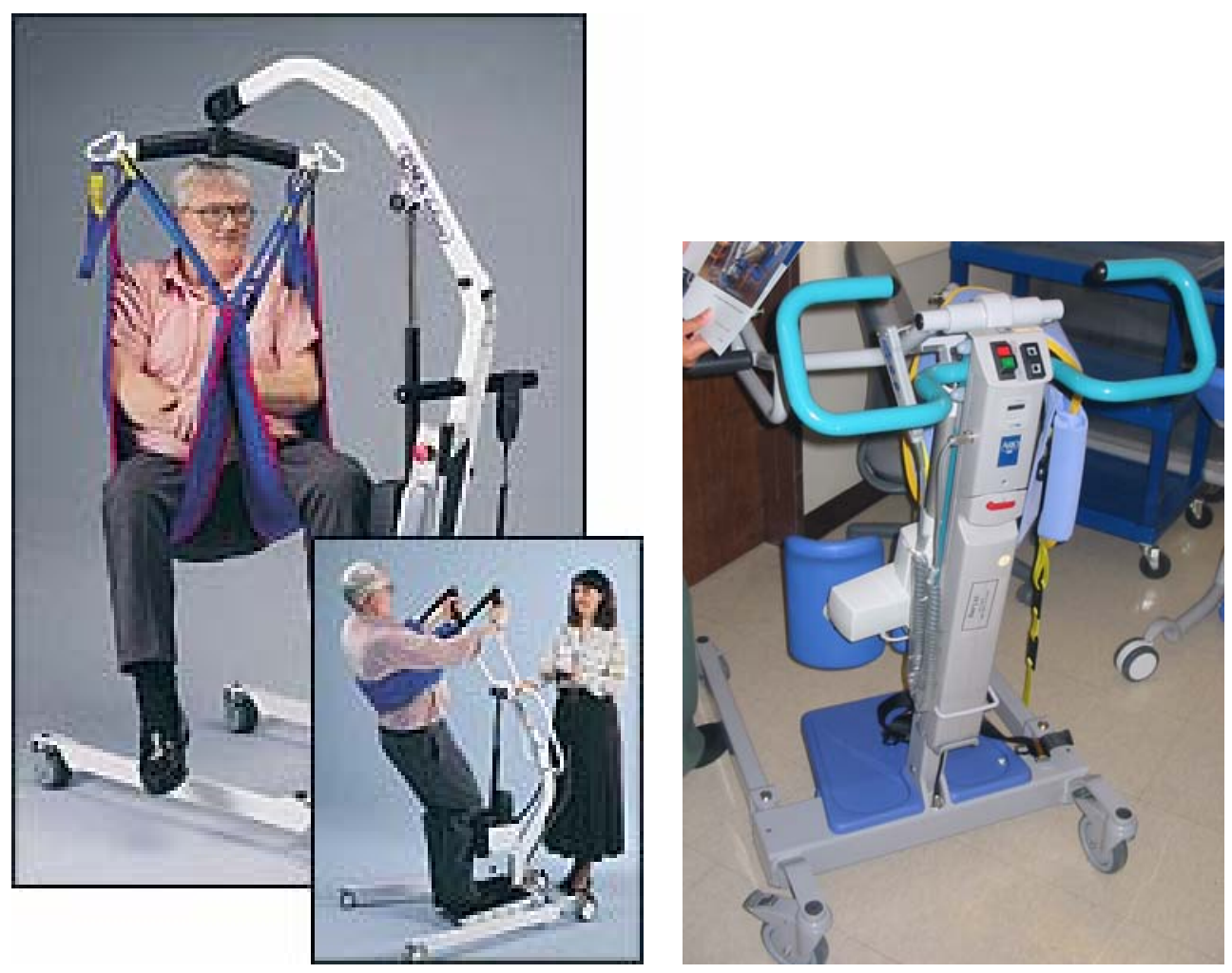

(thanks to UCSF)

This patient lift device is a very basic version of the one shown in the above, inset photo where instead, while the seat is folded up, the patient provides lift power from their upper body by grabbing the upper bar and pulling themselves up. The patient's shins are supported from moving forward and the seat is folded down to allow the patient to sit while the caregiver can mobilize the patient.

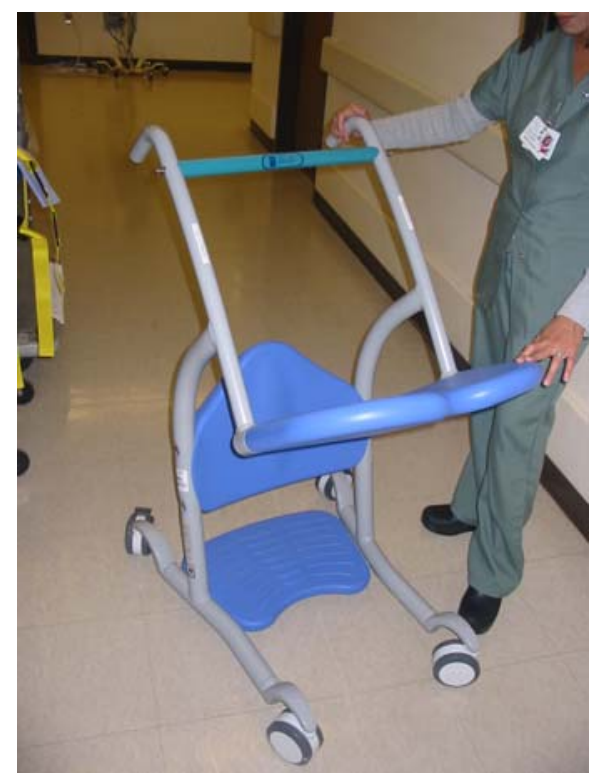

(thanks to UCSF) 
This powered lift seat/bed allows lift from a seated or laying position. The arm rest is movable allowing the patient to slide off the device on the open side. The device is however, blocking access to the patient from the lift control side.

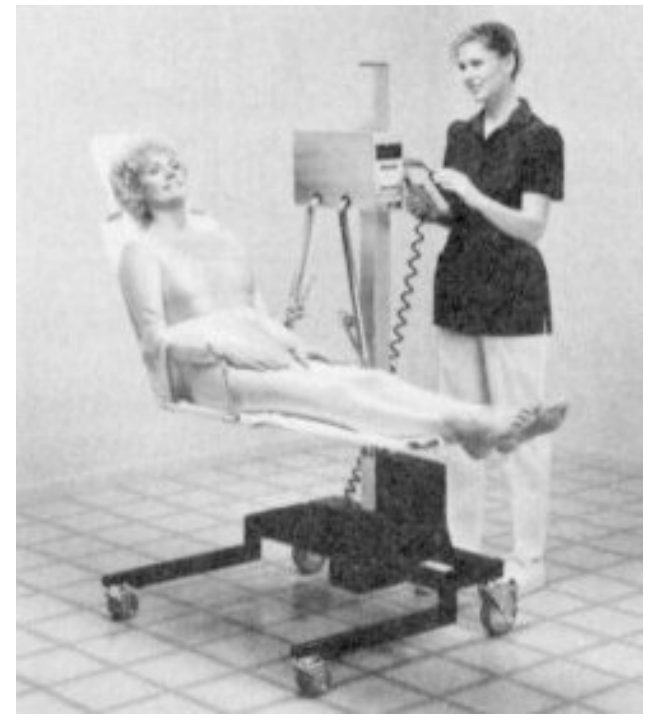

This lift device has two degrees of freedom allowing lift of a harnessed patient and rotation about a horizontal pivot mounted to the wall or other support frame, such as a bed.

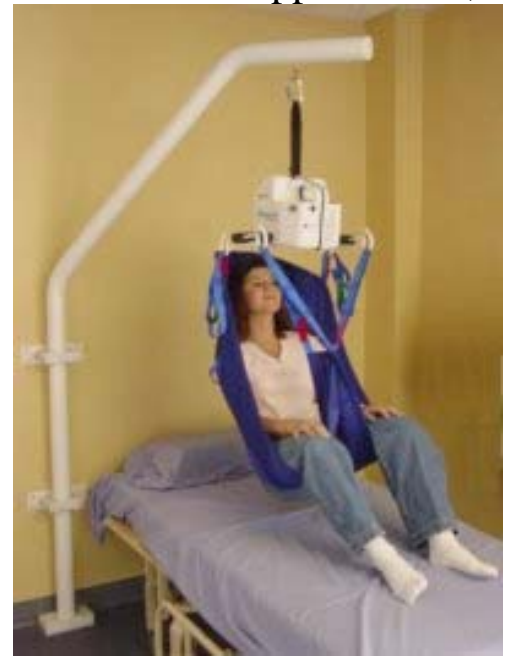

The Stepless Lifting Platform, model LP6, is an exterior wheelchair lift designed to lift individuals who use wheelchairs or who have walking disabilities over small height differences. Designed for use in situations in which ramps would be too steep or to long, this unit can be used as permanent or temporary installations. A choice of an electrically or manually operated ramp provides access to the lift and prevents the wheelchair from rolling off while the lift is in use. The electric ramp can be operated with a hand control for independent use, while the manual ramp requires attendant assistance. A safety lip with railings on the long sides and a non-skid surface on the platform and ramp provide additional safety. 


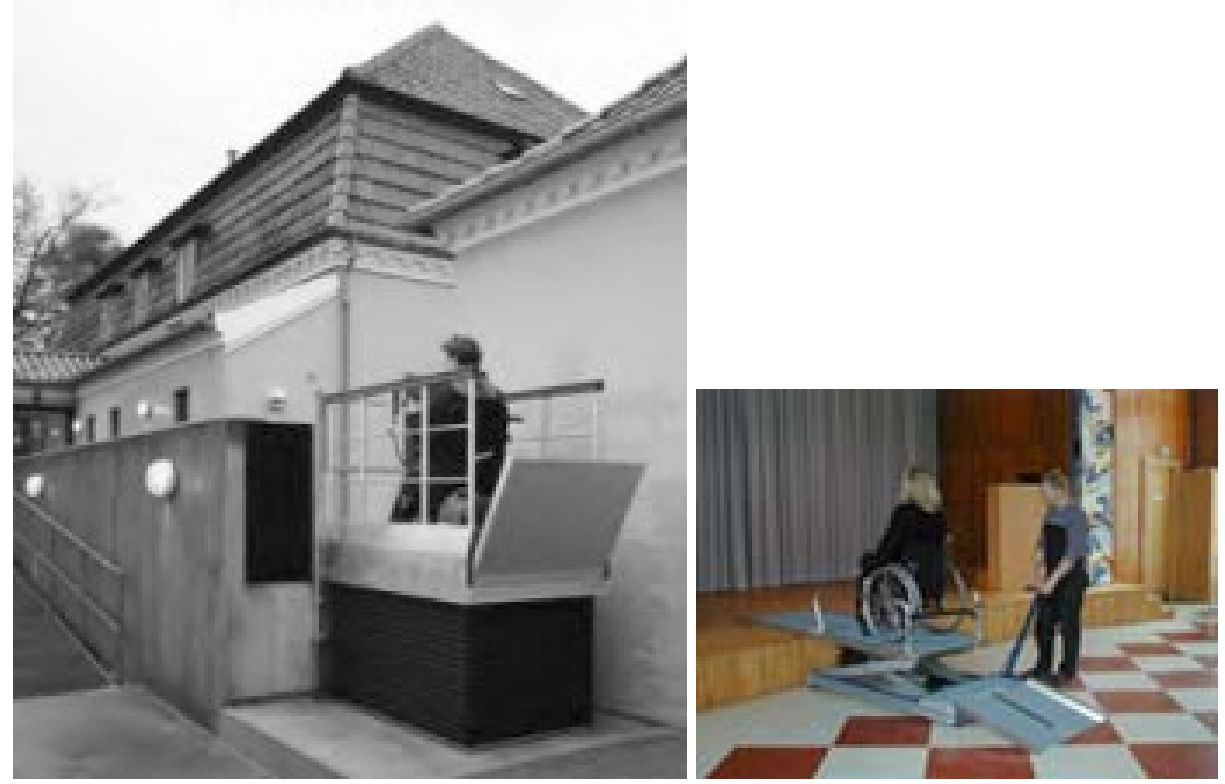

Bruno Independent Living Aids, Inc.- Lift-Up ${ }^{\mathrm{TM}}$ Power Mobility Seat

This device provides independent lift into and out of a vehicle with a powered seat. The patient however, needs to get themselves onto the seat from a walker, wheelchair or other device possibly requiring assistance from a caregiver. The device was designed exclusively for the 2004/2005 Toyota Sienna Minivan, where it provides safe, convenient driver/passenger transportation since it uses the actual seat from the vehicle including a standard safety seat belt.

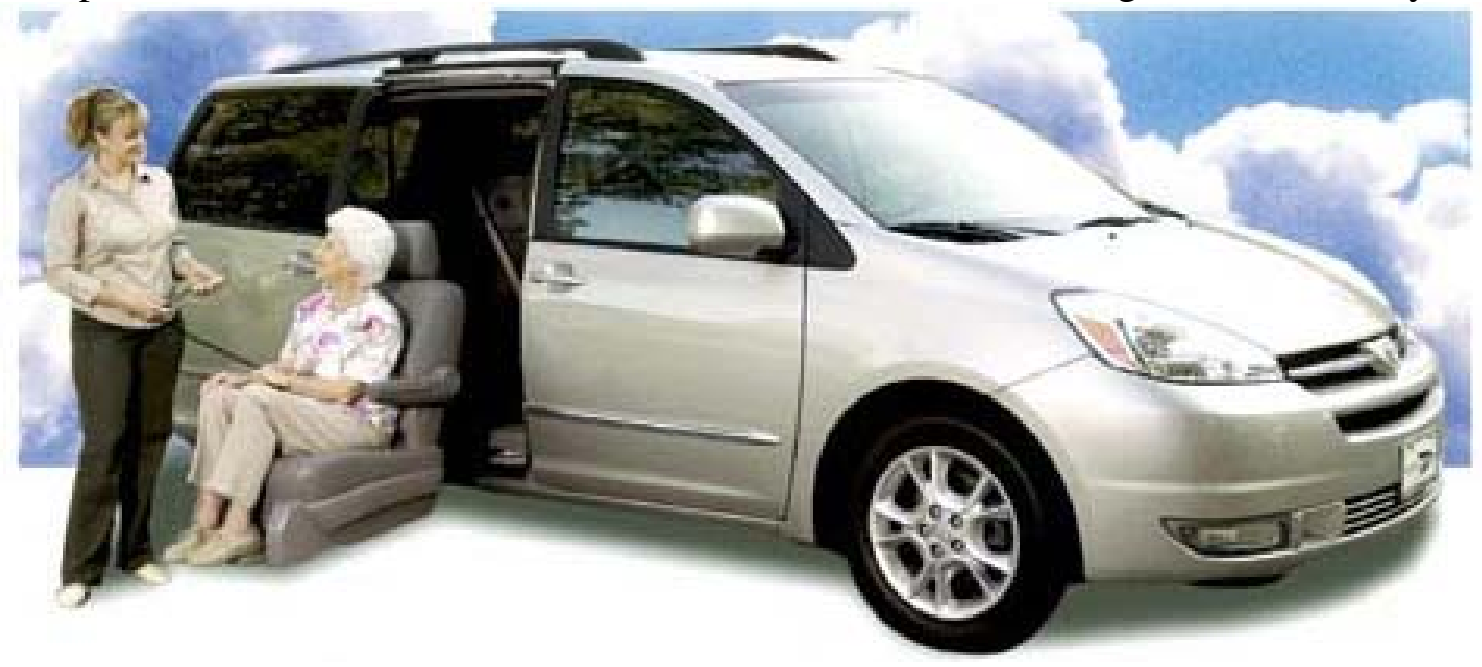

\section{Independent-Care Patient Lift Devices}

$\underline{\text { Trapeze-style lifts }}$

Trapeze style lifts are typically connected to beds or other supports to allow the patient with some upper body strength to lift themselves manually from a laying or seated position. 
Draft: 9/15/06

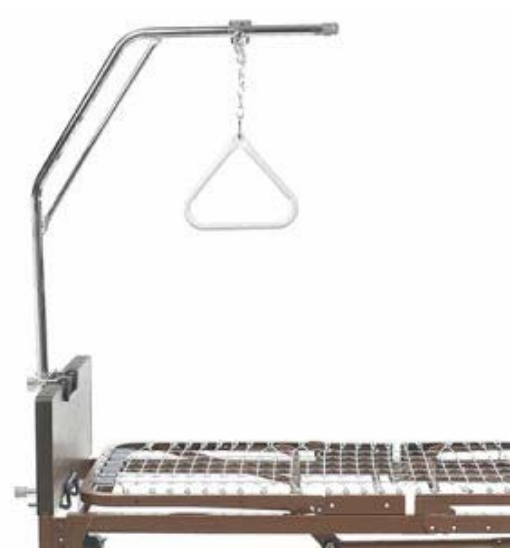

This powered lift device (left) is mounted to the ceiling providing lift and mobility from, for example a bed to a chair. The harness is required to surround the patient and therefore must be initially placed beneath the patient and attached to the ceiling lift. The stair lift (right) provides lift up a set of stairs from a seated position. It is mounted to the wall or stairs and power-lifts the chair along the steps. The patient must, however remove themselves from a wheelchair or walker, sit on the stair lift and get back off the chair at the stair top/bottom.
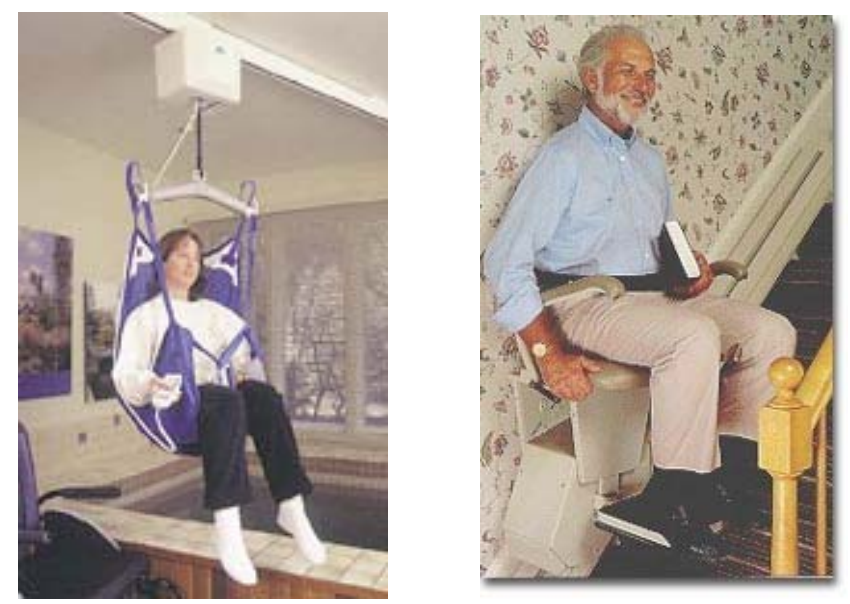

Frank Mobility Systems - Transborder
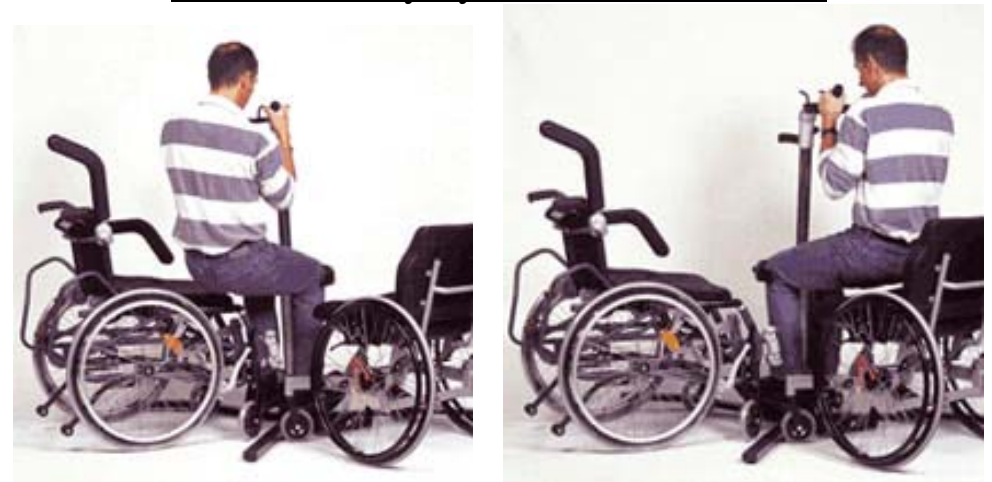
This device allows a person to move themselves from one see to another independently. The person pivots about the tripod-supported post with users feet on it's base. User upper body strength must support a large percentage of their weight.

\section{NIST - Lifter}

The Lifter is a concept designed to allow a person to be lifted and placed on a bed, chair, toilet or even floor. Mobility is self/caregiver-powered (walking), assist-power (optional - walk with some mobility power from Lifter), or powered (optional - full mobility power from Lifter). Powered requires additional drive motors not shown in the graphics. Also, for typical stroke victims where the right leg is not or partially functional, a fold down, removable footrest can be optionally attached to the right side. For full mobility, footrests for both feet can fold down. [Bostelman, 2004]

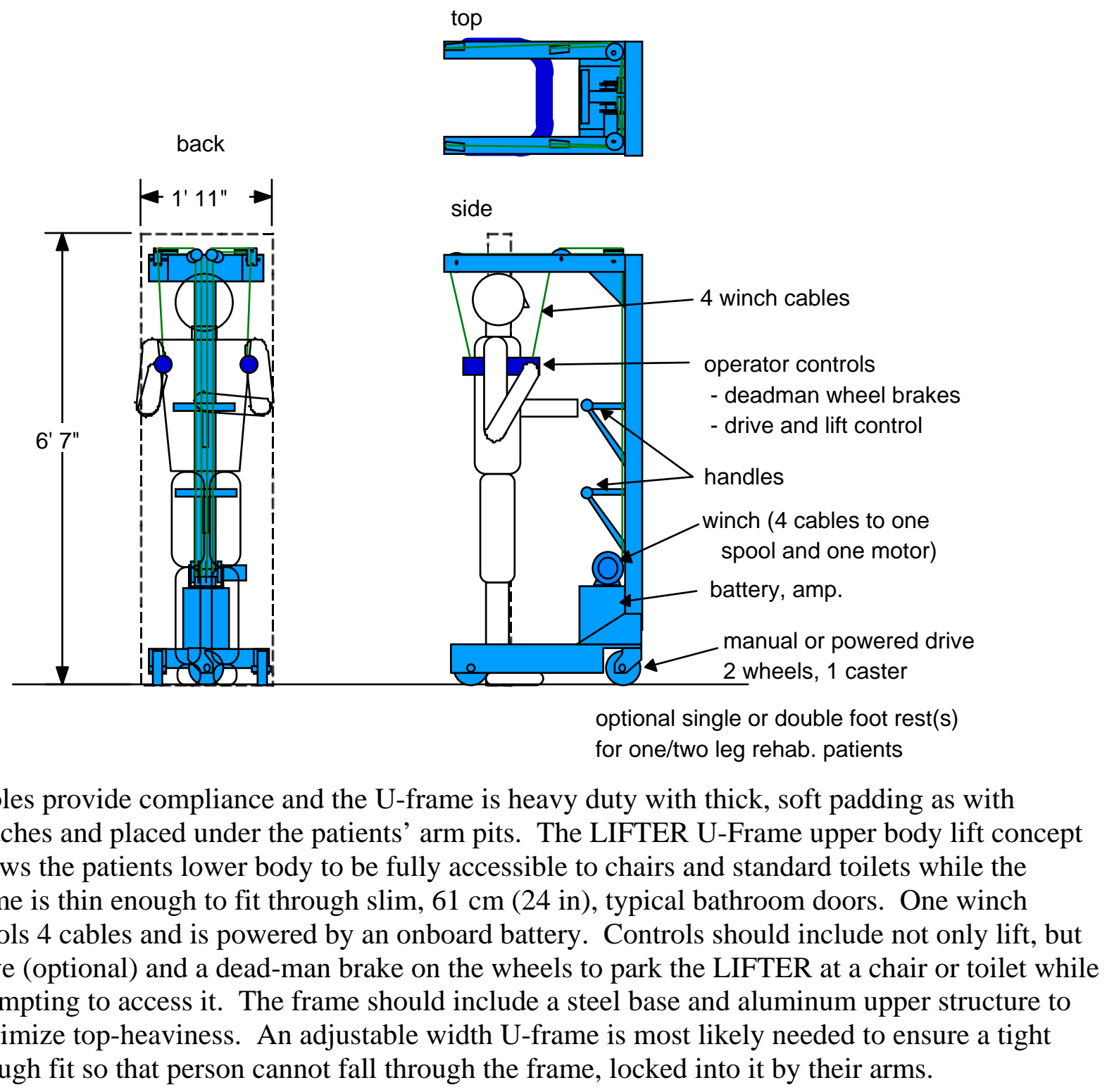




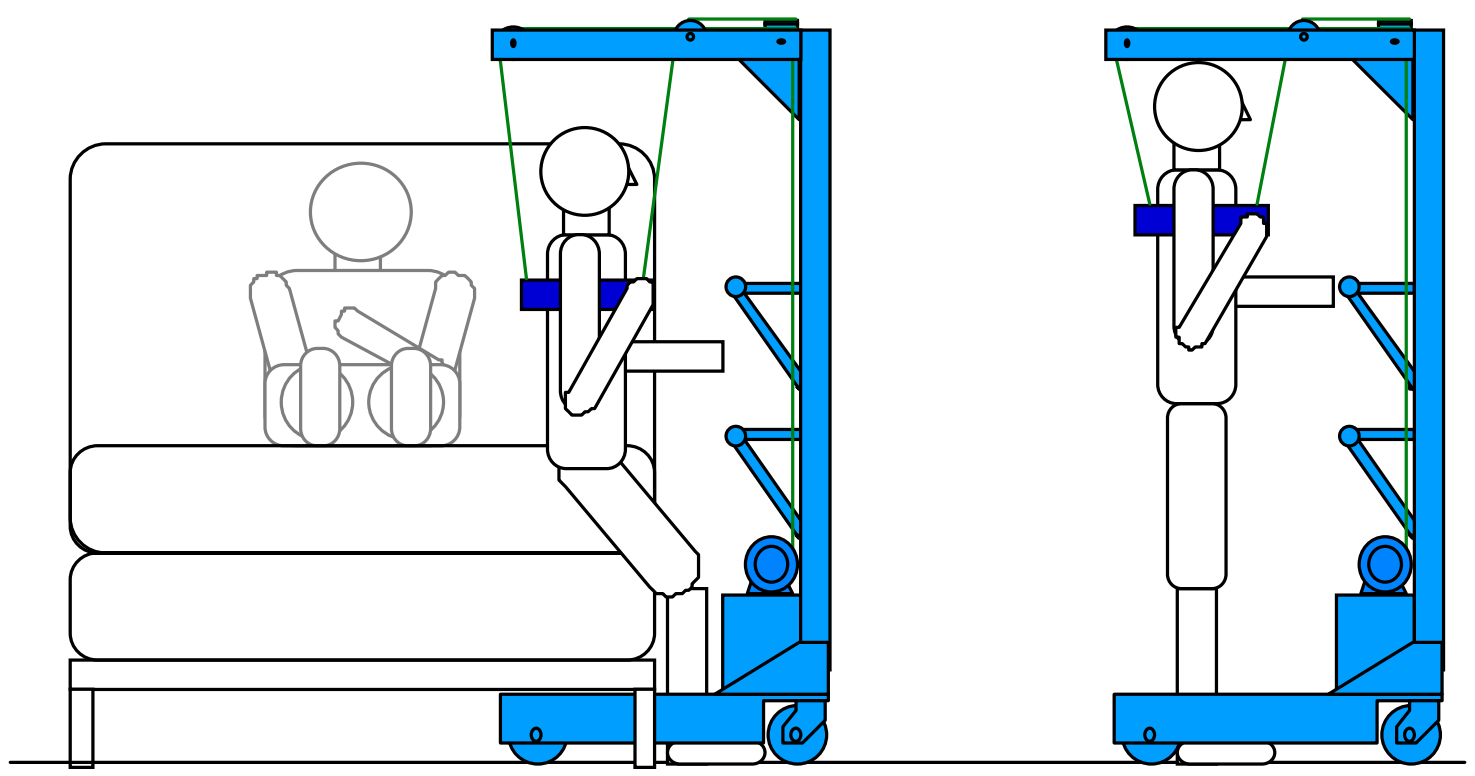

Access to bed (as shown), chair, toilet or even the floor is possible by using cables to lift and lower the person via the U-frame. All cables are spooled on one hoist to minimize required power. Surrounding cables also ensure the person will not fall backward out of the U-frame. Handles mounted at two locations allow for lower and upper access and provide push, pull and body control when mobile.

Crossing industry applications, the NIST Lifter can be used for manufacturing applications where a strap can be attached to the U-frame and placed around boxes, bulky and/or awkward objects for their lift. Lift the corner of the box, put the strap under and hoist the object up. A Cframe as in the healthcare design (left) or a Personnel-Platform, power-drive design (right) both seem feasible. In the Personnel-Platform design, the person supplies the necessary weight and determines, to a limit, how much load can be lifted. A load cell can ensure proper counterbalance. And retractable support wheels can ensure the load is never dropped or that the LIFTER can never fall forward should the person step off of the platform. The load can then be set on the extended support wheels for safety during mobility. The extension can be manually kicked forward by the operator. 

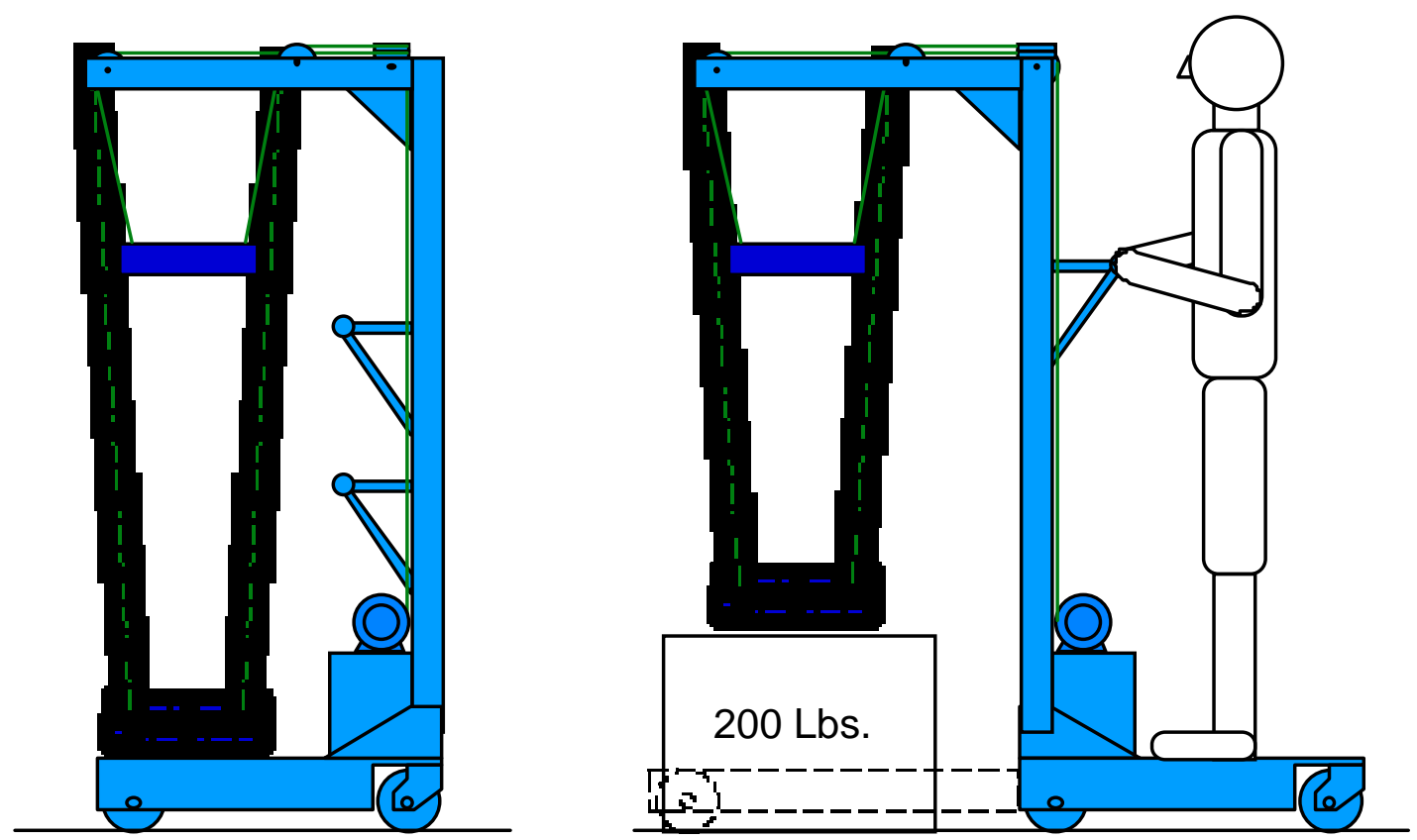

The wheelchair-to-vehicle lifter is an independent patient lift device that can lift a person sitting on a wheelchair or other seat and place them into a vehicle. It can be retrofit to most vehicles where the lift support arm attaches to the vehicle and the attached seat is strapped to the patient. The lifter seat therefore, rides with the patient even in the wheelchair and within range of the lift device, it mechanically attaches to the lift arm. A caregiver must however, stow the wheelchair.

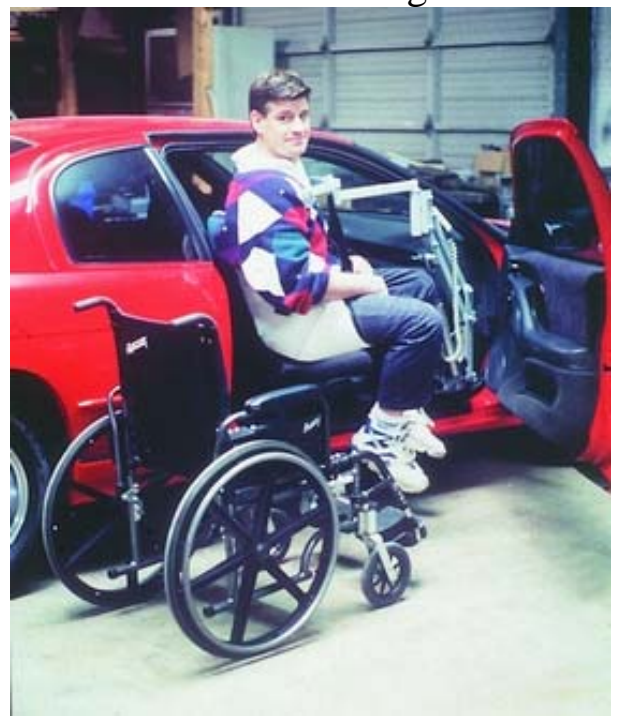

\section{Entervan}

This device is a pull-out ramp allowing a wheelchair to be rolled in and out of the vehicle where the wheelchair provides the seat for the patient while onboard the vehicle. The seat therefore provides no need to remove themselves from the wheelchair in order to enter, exit or even ride in the vehicle. However, it does provide a need for wheelchair safety holds within the vehicle during transport. 


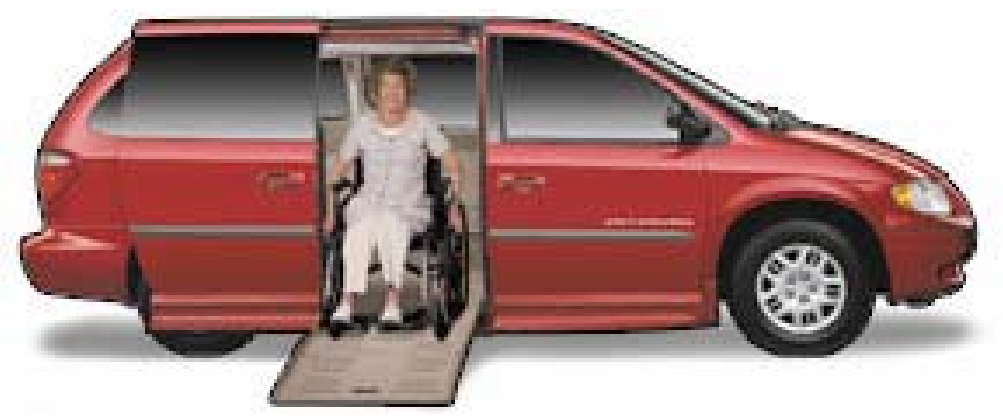

Toward the safety of such devices while riding in vehicles, the NIDRR/DOE provided \$4.5 M for a five year study on wheelchair safety during transport in other vehicles beginning in November 2001. The "Rehabilitation Engineering Research Center on Wheelchair Transportation Safety” represents a comprehensive collection of projects focused on improving the safety of those using their wheelchair as a motor vehicle seat. Projects address wheelchair securement, occupant crash protection, development of industry standards, and assessing the incidence of wheelchair user injury in a motor vehicle crash. [NIDRR/DOE, 2001]

\section{Manual Wheelchairs}

\section{WHEELCHAIRS}

Manual wheelchairs, like the example shown below in the photo, are: portable as they can fold to a smaller size, are relatively lightweight as compared to powered wheelchairs, have been around for hundreds of years as stated in the Introduction, and have become a pseudo-standard in hospitals, shopping malls, homes and many other facilities to assist immobile patients.

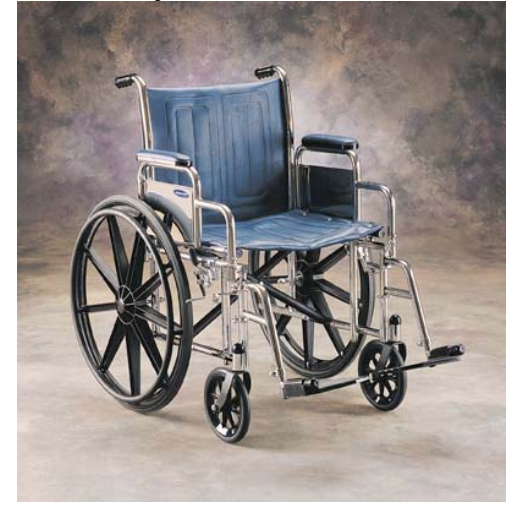

\section{Powered Chairs}

Like the examples shown below, powered chairs (left) and scooters (right) have become readily available on the market today and are made by several companies. These devices allow the operator to control their mobility without exerting manual force to move them and the device. Using one's arms to push a manual wheelchair can result in injuries, the need for surgery, and potential loss of independent mobility. [Cooper] Powered chairs can help eliminate these issues.

It is however, important to note the need for operator attention while driving powered scooters or chairs. The author personally watched an elderly person who recently suffered from a stroke driving a scooter while being inattentive to his surroundings and inside a store. As a result, several times the elderly driver crashed into store displays, shelving and other people. This dangerous situation begs for assistance from a caregiver to manually push this person around 
using a manual wheelchair. Alternatively, the powered chair could be equipped with advanced operator attention control interlocked to the low level mobility power to simply cut-off power to the drive system when the operator is inattentive. The Intelligent Wheelchair section of this report discusses face-tracking research that could support this alternative.
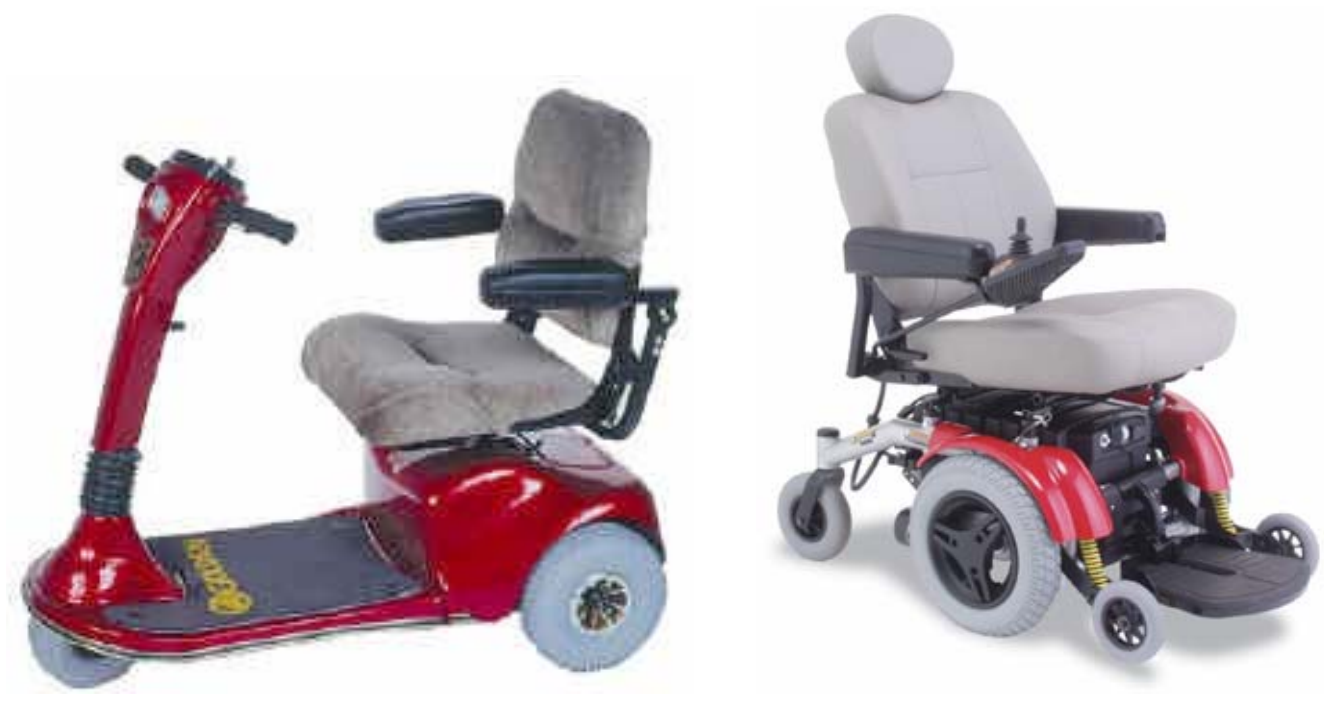

\section{NIDRR-RERC - Power Wheelchair Comparison Study}

A study comparing powered wheelchairs has recently been completed. Sections 1 through 10 and 14 have been completed on fifteen different wheelchairs. Information on the methods, results, discussions, and conclusions for each section can be found in "Analysis of the ANSI/RESNA Wheelchair Standards” on the University of Pittsburgh website. [Cooper, 2004 2]

Once the initial testing was completed, all of the power wheelchairs were then cycled through a two-drum and a curb drop machines until a class III failure occurred that made the wheelchairs inoperable. Descriptions of these standard class failures can be found in the NIDRR-RERC website. The best chair had a mean of 2,752,869 equivalent cycles while the shortest cycle had a mean of 276,981 equivalent cycles. The best chair also had the highest value (387 equivalent cycles per dollar), and lasted the longest until the first occurrence of a failure, as well as the number of equivalent cycles between failures, consumer repairs, and supplier repairs.

The number of individuals using electric powered wheelchairs (EPWs) is increasing every year. Advances in technology have led to the design of EPWs that are more complex and can perform multiple functions. The ANSI/RESNA wheelchair standards consist of a battery of tests that are designed to evaluate the safety and performance of both manual and power wheelchairs. However, there is a deficit of information available to the general public on the performance of wheelchairs on these tests. The purpose of this study was to compare the results of standards testing on five different types of EPWs. The value and intentions of each section of the standard were also reviewed and suggestions were made for possible improvements. A total of fifteen EPWs (three of each type) were tested using the following sections: static stability, dynamic stability, effectiveness of brakes, energy consumption, overall dimensions, speed and 
acceleration, seating dimensions, static, impact, and fatigue testing, climatic testing, obstacle climbing ability, and power and control systems. Statistical analysis was performed on the relevant sections. Significant differences were found between the different types of wheelchairs with respect to static stability, dynamic stability, braking distance, theoretical range, and obstacle climbing ability. The EPWs with the highest velocity and accelerations were found to be the most dynamically unstable and have the longest braking distances. Dynamic stability and braking distance were also found to be directly related to the slope of the test surface. It is apparent from the results that EPWs can differ in both performance characteristics and safety. Evaluation of the wheelchair standards also illustrated the need to continually revise the standards to keep pace with new technology. Stability, fatigue strength, and control system testing are three of the sections that will need to be adapted to help evaluate the next generation of EPWs.

\section{Power-Assist Manual Wheelchairs}

A study has also been done to evaluate power-assisting manual wheelchairs.

Using one's arms to push a manual wheelchair can result in injuries, the need for surgery, and potential loss of independent mobility. The purpose of this study is to evaluate the effectiveness of Yamaha’s JWII Pushrim Activated Power Assist Wheelchair (PAPAW) among manual wheelchair users with tetraplegia.

The study consists of three phases. For Phase I, the amount of energy required to push a manual wheelchair will be compared to a PAPAW. In Phase II, wheelchair users will evaluate the PAPAW over an Activities of Daily Living (ADL) course to determine its usability and acceptability. In Phase III, participants will take a PAPAW home with them for two weeks, and certain activities will be monitored. Fulltime manual wheelchair users with tetraplegia (those with spinal cord injuries at the cervical level or above) are being recruited to participate in the study. Currently the first two phases have been completed, and participants are currently being recruited for Phase III of the study.

Phase I results indicate that when using the PAPAW, individuals showed a significant decrease $(\mathrm{p}<.05)$ in both the number of strokes needed to maintain target speeds and upper extremity joint range of motion across all but a few trials. This reveals that, when using a PAPAW, an individual is pushing less often and with less range of motion than a standard manual wheelchair and, thereby decreasing the risk for developing RSIs and upper extremity pain. Furthermore, subjects showed a significant decrease $(p<.05)$ in heart rate, the amount of oxygen consumed, and the amount of air expired when using the PAPAW. Results from the second phase of the study reveal that participants preferred the PAPAW to their own wheelchair when attempting to complete a number of obstacles over the ADL driving course. In addition to this, their overall heart rate was significantly decreased $(\mathrm{p}<.05)$ when using the PAPAW to complete the driving course.

PAPAWs can be particularly useful for elderly wheelchair users with tetraplegia, because upper extremity weakness is typically found in this population. PAPAWs have the potential to reduce stress on upper extremities during wheelchair propulsion, reduce metabolic energy expenditure, 
improve function during daily activities, and improve mobility and participation within the one's community. [Cooper, ????]

\section{Cooper - Smart Power Assisted Module (SPAM)}

The concept of power assistance for a manual wheelchair is relatively new, and represents a viable alternative for individuals who are unable to generate sufficient propulsion force to use a manual wheelchair, but do not wish to use a traditional powered mobility device. In a power assisted manual wheelchair, the traditional rear wheel hubs are replaced with motorized hubs that serve to magnify or reduce (i.e., brake) the propulsive force applied to the rear wheels by the user. We propose to use power assistance as the basis for a Smart Power Assistance Module (SPAM) that provides independent mobility to non-ambulatory individuals with visual impairments. [Cooper, 2004]

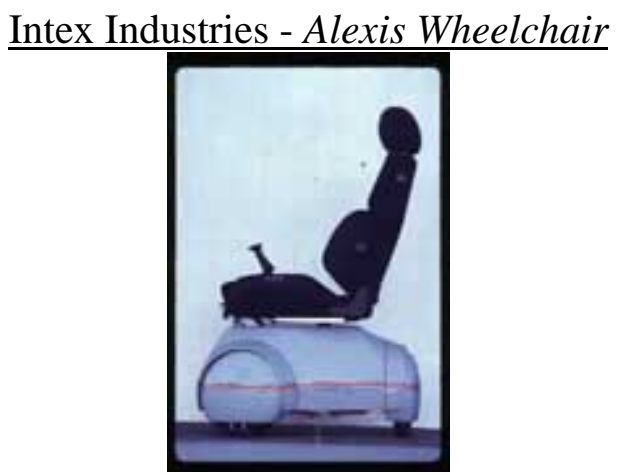

Alexis is an innovative electric wheelchair using a "wheels within wheels" design. It is unique in that it can turn in its own footprint and move sideways. The Rehab R\&D Center licensed Intex Industries to make Alexis commercially available in 1987, and Intex made 40 pre-production units for field trials in the San Antonio area. During subsequent redesign efforts, the company filed for bankruptcy, preventing further commercialization at this time. [Stanford, 1987] http://guide.stanford.edu/Projects/CommlProd.html

\section{Intelligent Wheelchairs}

\section{AAAI - Health Care / Elderly}

“The world's population is rapidly aging, and some are predicting a coming 'crisis in caregiving.' Intelligent technologies have the potential to help avoid this crisis by providing people with ways to adapt to the physical and cognitive changes that can accompany aging."

A number of global wheelchair projects have developed representing a thrust toward assisting the rider with navigation, obstacle avoidance, docking, climbing curbs and stairs and other necessary tasks typically provided by a caregiver. Without the care giver, the same tasks are required by the chair itself. A sample of several intelligent wheelchair projects are shown below and listed in the Reference section that address these and other issues adding intelligence to the wheelchair. Although not exhaustive, the projects shown provide a direction of this research with only minimal intelligent lift-wheelchair developments. What appears to be missing is a generic means typically provided by the care giver to lift the patient from bed or a seat, deliver them to the wheelchair, guide/push them to another location, remove them from the wheelchair, and place them on another seat. This is a very manual labor intensive and potentially traumatizing set of tasks for the care giver and patient. Ideally, the chair will provide all means 
of seat-to-seat capability.

\section{ActivMedia Robots - RoboChariot}

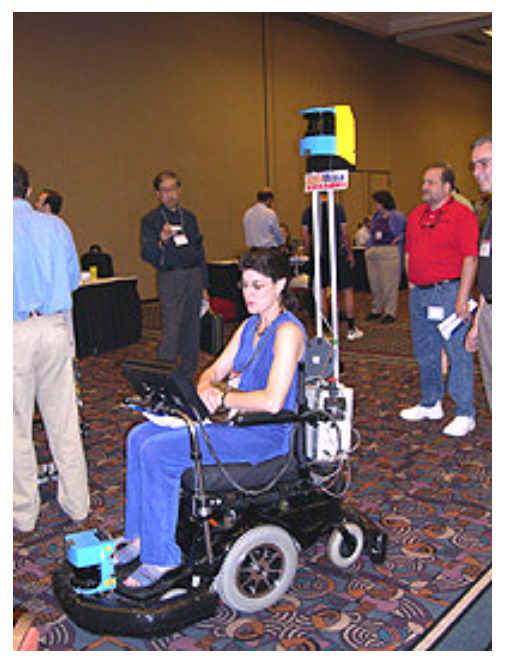

ROBOTIC CHARIOT is based on a Pride Mobility Jazzy 1120 power chair integrated with ActivMedia Robotics Control Systems (ARCS) including Laser Mapping \& Navigation and ARIA software-compatible with all ActivMedia robots. Chosen by those requiring indoor and paved outdoor terrain capabilities, 6pmh speed and plenty of real estate for customization. Powerful, yet easy to use; reliable, yet flexible, Robotic Chariot is a popular performer for wheelchair, transport or other vehicle research.

The Robotic Chariot includes an embedded computer, joystick/LCD or touchscreen interface, Ethernet-based communications, laser mapping and navigation, and bump sensing. The Robotic Chariot stores up to 252 watt-hours. Robotic Chariot's powerful motors and 2 drive-wheels can reach speeds of $7 \mathrm{kph}$ and carry a payload of up to $100 \mathrm{~kg}$. The Robotic Chariot includes shaft encoders with gyroscopic correction to compensate for radial dead reckoning errors. Options include:a second laser on a sliding pole for localization in crowded spaces.

The Robotic Chariot base with included control software has the ability to:

- WANDER randomly

- DRIVE controlled by keys or joystick

- PLAN PATHS with gradient navigation

- DISPLAY a map of its laser readings

- LOCALIZE using laser

- COMMUNICATE SENSOR \& CONTROL information relating shaft encoder readings auto-corrected by gyroscope, motor controls, user I/O, and battery charge data

- $\quad$ TEST ACTIVITIES QUICKLY with ARIA API from C++ programs

- SIMULATE BEHAVIORS OFFLINE with the simulator that accompanies each development environment

The Robotic Chariot is an all-purpose wheeled chair, used for research and prototyping applications involving:

- mapping 
- navigation

- delivery

- and other behaviors

Robotic Chariots's are made for use indoors or outdoors; they run on carpet, floor, packed earth and paved surfaces. They can climb 15\% grades with full payload. The control systems (PC and other electronics) are not water-proof.

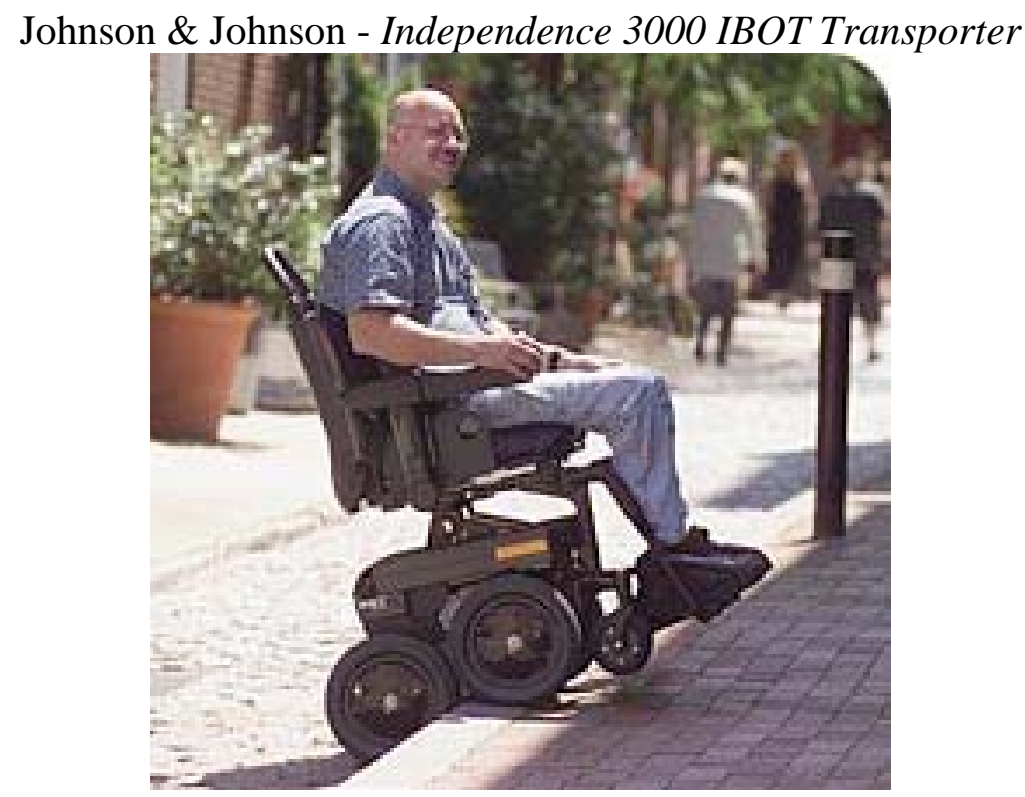

- Climbs curbs, stairs

- Traverses sand, grass

- Raises up on two wheels for eye-level mobility

- $\quad$ Standard, 4-Wheel, standing, and stair climbing modes

$\underline{\text { Palo Alto VA Health Care System -Ultrasonic Head Control Interface }}$

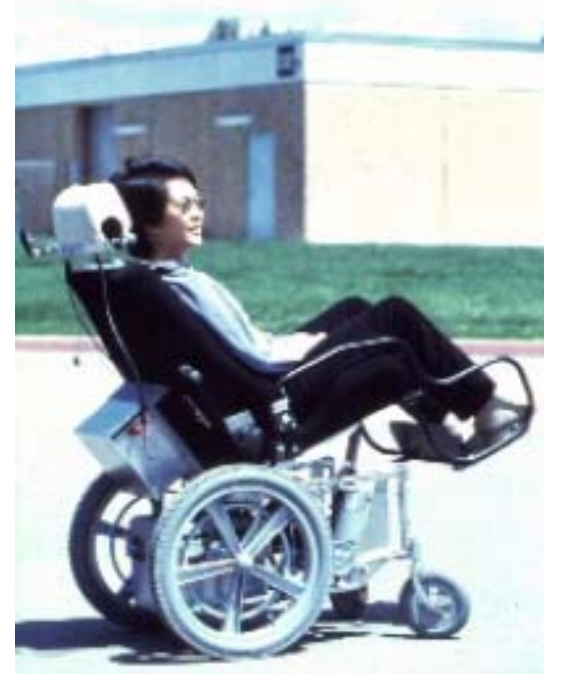


Features

- 2 degree of freedom real-time operation

- non-contact interface

- front or rear sensing

- mouse or joystick substitute

_ Applications

- control of mobility (electric wheelchair) contrast with voice control alternative

- control of cursor position with hands on keyboard

- demonstrated robot control

Commercialization effort

- wheelchair operation approved for VA use

-working with company for commercialization

Communication Aids for Language and Learning (CALL) Centre - Smart Wheelchair

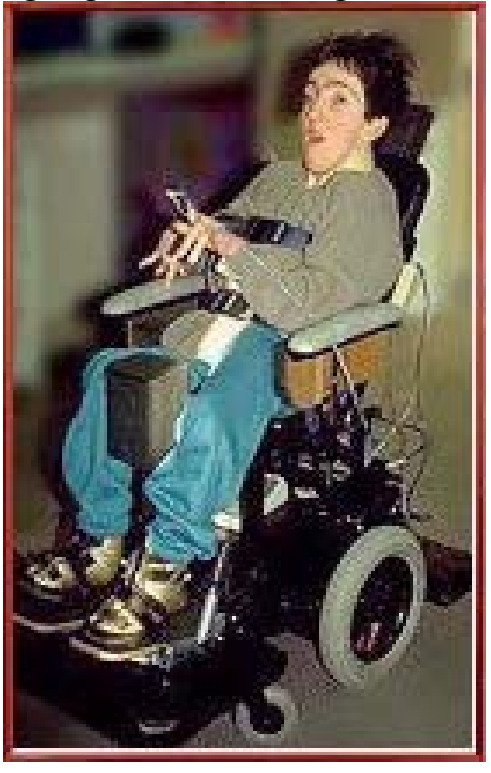

Collision Sensors, Line Follower and Feedback

The basic Smart Wheelchair is based on a commercially available powered wheelchair chassis, controlled using a computer designed and built by the project team. The chairs are intended to be used by children who do not have the physical, perceptual or cognitive abilities to control an ordinary powered mobility aid, and so there are a number of features to protect the user and environment and enhance the activities possible with the chair.

The CALL Centre (Communication Aids for Language and Learning) provides specialist expertise in technology for children who have speech, communication and/or writing difficulties, in schools across Scotland.

The CALL Centre originally developed the Smart Wheelchair for children with severe and multiple disabilities who could not use ordinary mobility aids. With the chair, children experience new opportunities for communication, learning, exploration, play and achieve some degree of independent mobility. Collision sensors can stop the chair on contact with an obstacle, 
and if desired reverse and also turn it away from the object. Ultrasonic rangefinders are under development to slow the chair prior to collisions, and to help with negotiating corridors and doorways. A line follower lets the chair follow a track laid along the floor, to help pilots move from room to room or negotiate difficult situations such as doorways. Finally, the chair confirms instructions and reports events back to the user via a speech synthesizer or other feedback technique. [CALL Centre]

The Smart Wheelchair is built on a compact power base. Virtually any seating system can be fitted to it, such as a CAPS II. A Controls Dynamic DX control system gives excellent control and maneuverability. The Smart Controller acts like a second DX joystick, and the various Smart systems and controls plug into the Controller. The Smart Wheelchair 'tools' can be easily selected in different combinations to suit the pilot and environment.

The chair can be driven directly with one, two, three or four switches, joysticks, or a scanning direction selector. The scanner can be operated with one or two switches, and can even speak out the directions (auditory scanning) as they are offered.

The Smart Wheelchair can be driven by a laptop computer running programs like Clicker, or a communication aid, such as a DynaVox, via the RS232 port. There is a 'swap switch' facility for controlling the chair and communication aid form the same switch.

The chair's movements can be either:

* Momentary (go while I press the switch) - good control for good switch users

* Timed (go for a set distance) - for drivers who cannot hold down the switch

* Latched (go until I press the switch again) - for those with good switch press and release skills

The Smart Wheelchair has warp-round bumpers with zoned pneumatic sensors that detect collisions. The chair can respond in several different ways:

* Bump and Stop detects the collision and stops the chair

* Bump and Backoff stops the chair then reverses away from the obstacle

* Bump and Turn stops, backs off, then turns the chair to a different direction

* The speeds and distances, and direction and angle of turn, can all be configures for the user and situation

An infra-red track follower is fitted to the Smart Wheelchair, which lets it follow reflective tape stuck to the floor. Tracks can be easily laid around classrooms, schools and homes. The tape is tough and will stick to most surfaces including wood, vinyl and shallow pile carpet. The basic Line Follower lets the pilot follow tracks made with reflective tape on the floor from room to room, or helps them negotiate narrow doorways and tight corners. Line Following with junctions lets the pilot choose tracks at junctions. Tape can also be laid round dangerous features, like stairwells, to stop the chair reaching them. The speech synthesiser can confirm commands back to the pilot ("I'm going forward”), offer choices at track junctions ("Which way now - left or right?”), and report events (“Oops, I’ve hit something - I'll back off and try going left”). All the speeds, settings and Tools can be selected and adjusted with an easy-to-use 'ToolBox'. 


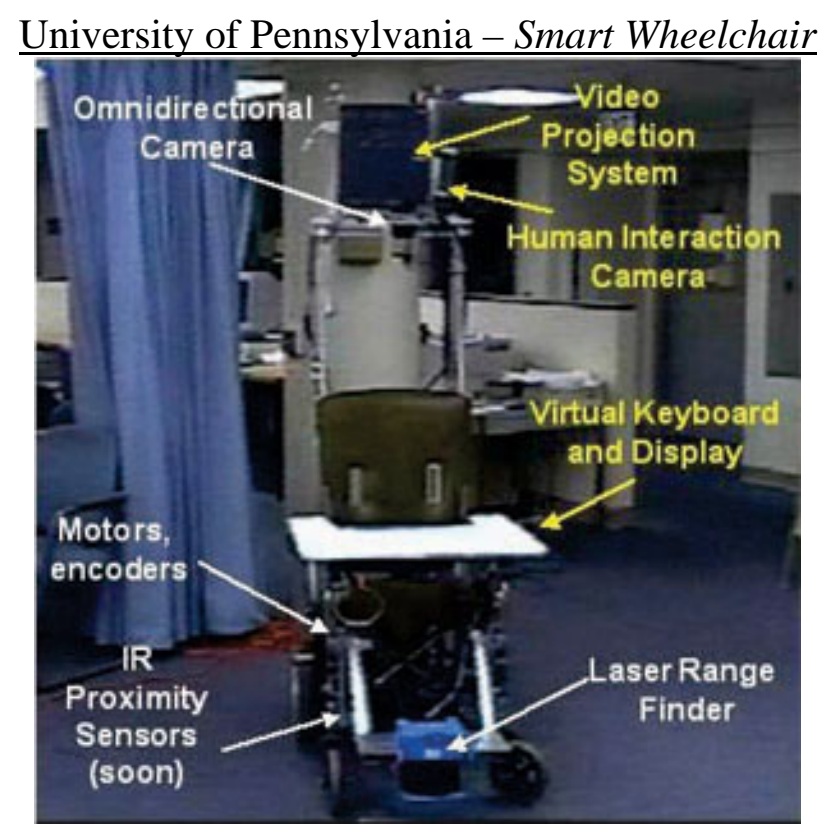

This paper [Patel, 2002] presents a sensor-based algorithm for guiding a nonholonomic platform, such as a wheelchair, through a doorway. The controller uses information from a camera system and a laser range finder to perform image-based navigation. Simulations of the resultant switching controller are presented along with experimental results. A simple obstacle avoidance algorithm is also implemented on the experimental platform. Finally, we have considered the input of limited field-of-view constraints on this controller. All of these components together lead to a modal, image-based approach that will safely and robustly navigate a nonholonomic robot with sensor constraints through a doorway.

Patel outfitted a motorized wheelchair with onboard processing and a suite of sensors as seen in the figure above. The omni-directional camera, mounted over the user's head, allows the user to view 360 degrees around the wheelchair. The projector system displays images onto the laptray and enables the user to send commands to the wheelchair through a visual interface. The laser scanner, mounted between the feet, measures distances over a 180 degree range.

Intelligent Wheelchair Remotely Controlled by Interactive Gestures

We presented an intelligent wheelchair whose motion can be controlled by the user's face direction. In this paper, we propose to add intelligence to our wheelchair when the user is not riding. It can recognize the user's face and can move according to the gestures made by the user. Gesture is a good means to give commands because it can be used in noisy conditions and we would not like to speak loud in some public places. However, environments where wheelchairs are used cannot be controlled. This makes gesture recognition difficult. We propose an interactive way to solve this problem. When the wheelchair is not certain about the meaning of user's gesture, it guesses the meaning and moves a little accordingly to show its guess to the user. Then it observes the user's response, judging whether its guess is correct or not. This guessaction-observation cycle is repeated until the wheelchair can understand the user's intention. [Kuno, 2000]

\section{Wellesley College - Wheelesley}




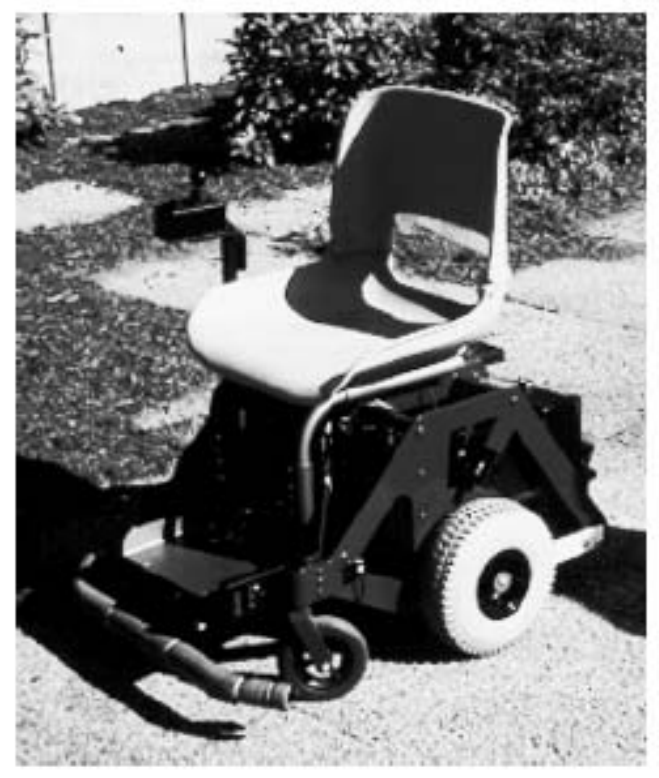

Figure 3 Wheelesley, the robotic wheelchair system.

This chair was built by KISS Institute for Holly Yanco of Wellesley College. She and her students designed navigation software and a GUI for the chair. The chair was demonstrated at the 1995 IJCAI Robot Exhibition.

Yanco's Ph.D. research addressed the creation of a robotic wheelchair system, named Wheelesley. In order for a robotic wheelchair to be useful, it must navigate effectively in indoor and outdoor environments, select modes automatically for its user and have a user interface that can be adapted for many different access methods. The robotic wheelchair system described in this paper, Wheelesley, automates many common navigational tasks for the user. The system consists of a standard electric wheelchair with an on-board computer, sensors and a graphical user interface running on a mounted notebook computer. [Yanco 1]

Massachusetts Institute of Technology Boston College - Single Switch Scanning Control Single switch scanning is the access method of last resort for powered wheelchairs, primarily because drift is a significant problem. To correct a drift to the left or the right, the user must stop going forward, wait for the scanning device to get to the arrow for the direction of choice, click to turn the chair, stop turning, wait to scan to forward and then click to move forward again. Robotic assisted control can improve the ease and speed of driving using single switch scanning. Under robotic control, sensors are used to correct the drift problem and to avoid obstacles. The user is only required to give commands to change direction, for example "left" at an intersection. [Yanco 2] 


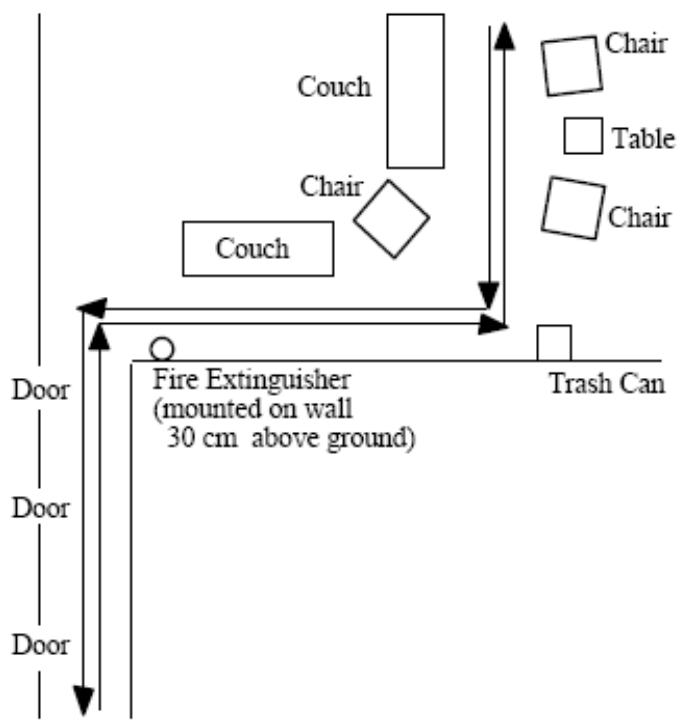

The graphic above shows a diagram of a test course. Subjects drove the course four times in each direction, alternating driving methods after a round trip of the course. The course was $20 \mathrm{~m}$ long, measuring along the outside edge of the course. Obstacles are couches, chairs, a table, a trash can, and a fire extinguisher mounted on the wall $0.3 \mathrm{~m}$ above the floor. The three doors in the hallway were open or closed, determined by the office occupants.

\section{Institute of Computer Science, Foundation for Research and Technology (ICS-FORTH) - Semi-} autonomous Navigation of a Robotic Wheelchair

The present work considers the development of a wheelchair for people with special needs, which is capable of navigating semi-autonomously within its workspace. This system is expected to prove useful to people with impaired mobility and limited fine motor control of the upper extremities. Among the implemented behaviors of this robotic system are the avoidance of obstacles, the motion in the middle of the free space and the following of a moving target specified by the user (e.g., a person walking in front of the wheelchair). The wheelchair is equipped with sonars, which are used for distance measurement in pre-selected critical directions, and with a panoramic camera with a 360 degree field of view, which is used for following a moving target. After suitably processing the color sequence of the panoramic images using the color histogram of the desired target, the orientation of the target with respect to the wheelchair is determined, while its distance is determined by the sonars. The motion control laws developed for the system use the sensory data and take into account the non-holonomic kinematic constraints of the wheelchair, in order to guarantee certain desired features of the closed-loop system, such as stability. Moreover, they are as simplified as possible to minimize implementation requirements. An experimental prototype has been developed at ICS-FORTH, based on a commercially-available wheelchair. The sensors, the computing power and the electronics needed for the implementation of the navigation behaviors and of the user interfaces (touch screen, voice commands) were developed as add-on modules and integrated with the wheelchair. [Argyros, 2002]

Korea Advanced Institute of Science and Technology (KAIST) - RTAI Based Real-Time Control of Robotic Wheelchair 


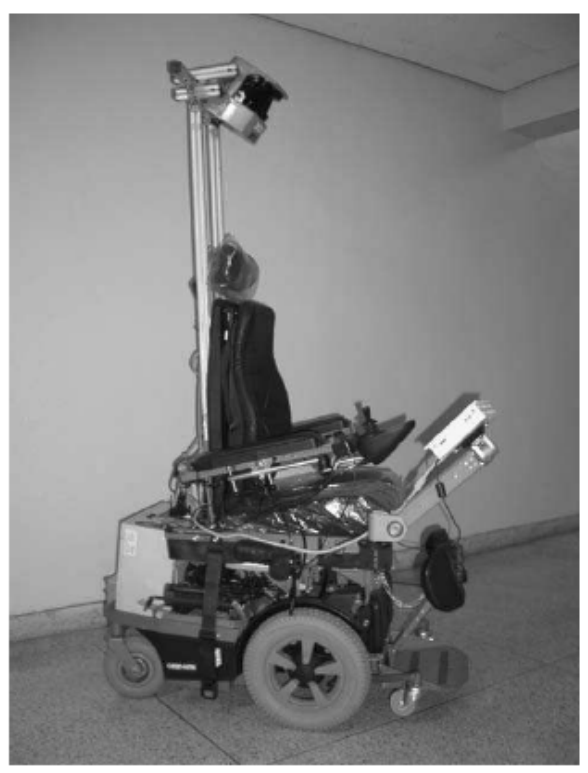

FIGURE 1: Robotic Wheelchair Platform

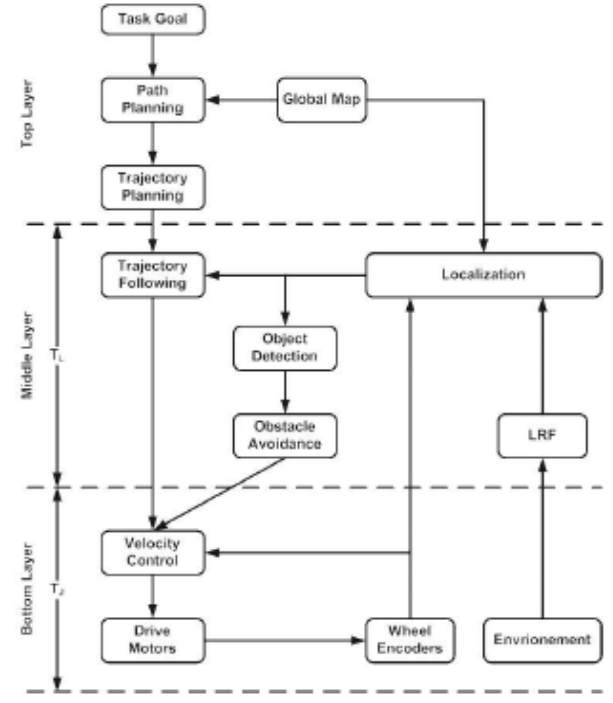

FIGURE 4: Hierarchical control architecture of our system

In order to provide autonomous mobility, a robotic wheelchair requires various tasks such as sensing, localization, obstacle detection, and motor control, which is a very complex control system. In this paper, we present a real-time control system design for robotic wheelchairs based on RTAI. We developed a multiprocessor-based robotic wheelchair platform for the disabled. Based on discussions on real-time requirements of robotic wheelchairs, we designed an efficient software architecture for autonomous real- time control of robotic wheelchairs. The performance is revealed by experimental results carried out at the Intelligent Sweet Home in KAIST.

The ultimate goal of robotic wheelchair is to take the user automatically and safely to the destination. In order to achieve this goal, many robotic wheelchair projects have been emerged in recent years. As representative research projects, there are NavChair [5], SIAMO [6], SENARIO [7], RobChair [8], MAid [9], FRIEND [10], Rolland [11], VAHM [12], and KARES [13]. Many research groups $[6,10,13]$ developed a robotic wheelchair under Windows operating system. [Kim]

We tested our developed robotic wheelchair at Intelligent Sweet Home in KAIST. Test objective is to transfer the disabled from a bed to the robotic wheelchair without assistance of another person. For accomplishing this objective, the robotic wheelchair moves autonomously to the predefined docking ready position and performs docking with the robotic transfer system. During docking operation, bumpers of robotic wheelchair detect collision with robotic transfer system and help safe docking. Figure 9 shows the robotic wheelchair is moving toward to the predefined position. During autonomous moving, robotic wheelchair performs localization, object detection, and motor control task. 


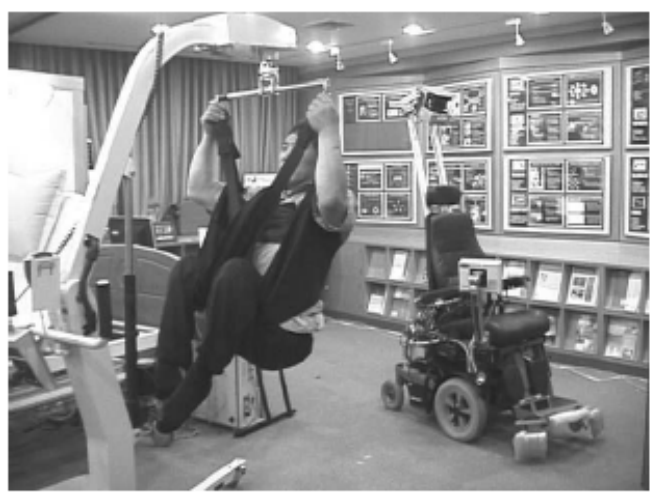

FIGURE 9: Autonomous Moving to the Predefined Position

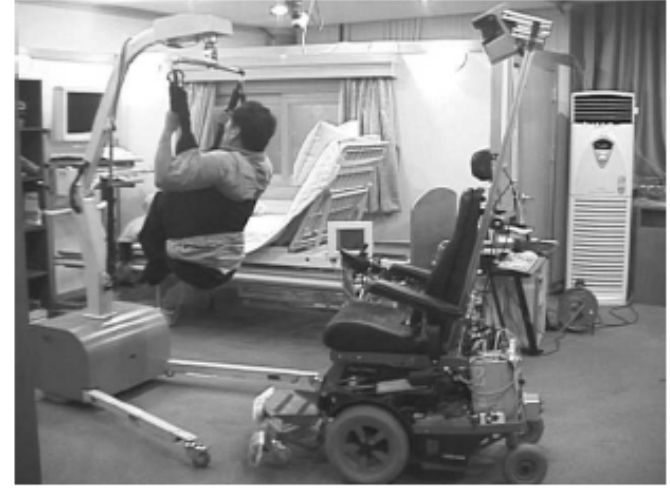

FIGURE 10: Docking Operation

KAIST - KARES: Intelligent wheelchair-mounted robotic arm system using vision and force sensor

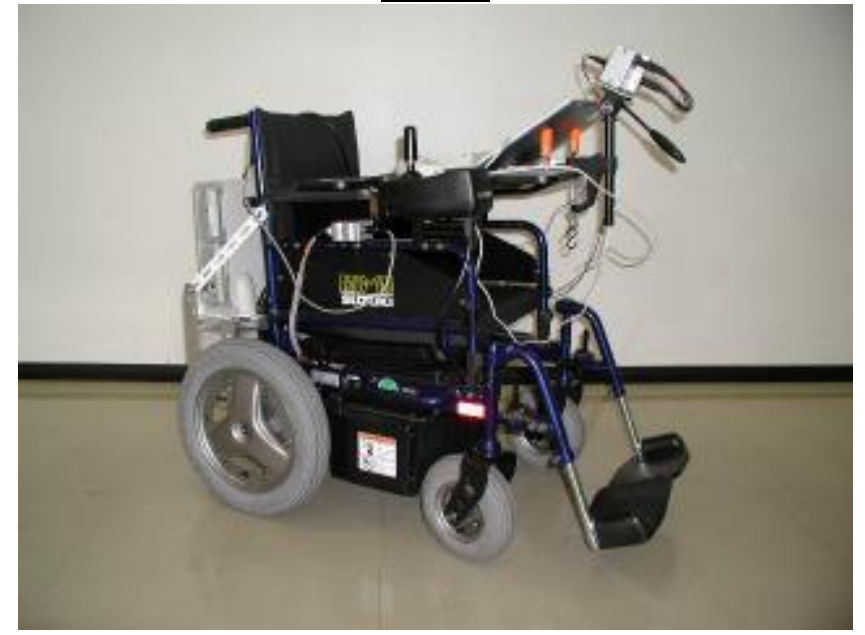

KARES, an acronym of KAIST Rehabilitation Engineering System, is the intelligent rehabilitation system with a 6 DOF robotic arm mounted on the powered wheelchair. It is developed to assist the disabled and the elderly for the independent activities. Human-machine interaction is a key issue of design for intelligent systems such as KARES. A special attention is paid in bestowing certain degree of autonomy to the robotic sub-system since the direct control of the robotic arm takes a high cognitive load on the user part while physically disabled persons may have difficulties in dexterously operating a joystick or push buttons for delicate movements. To perceive environment, one color vision sensor and one force/torque sensor are mounted on the end-effector of the robotic arm of KARES. To test the system, four basic tasks are defined as picking up a cup on the table, picking up a pen on the floor, moving an object to the user's face, and operating a switch on the wall. These tasks are performed autonomously in a semi-structured environment. [Song, 1999]

The Wheelchair is an important way of transfer for handicapped and aged people. Many researchers have been developing intelligent wheelchairs due to the increasing requirement of safer and more comfortable wheelchairs. We are developing a wheelchair which can be controlled by using both face direction and gaze direction. Owing to our robust face and gaze direction measurement system, it can run at both indoor and outdoor environments. 
The intelligent wheelchair is composed of following items:

- Suzuki Electric Wheelchair MC15S

- Board camera x 2

- Field Multiplexing Device

- Image Processing Board IP5005

- DOS/V PC (PentiumII 450MHz)

- Battery

\section{Control:}

- can control the motion of the wheelchair by using both face direction and gaze direction.

[Indoor Experiment](2000/Apr/21)

Screen shots of face tracking at various illumination conditions
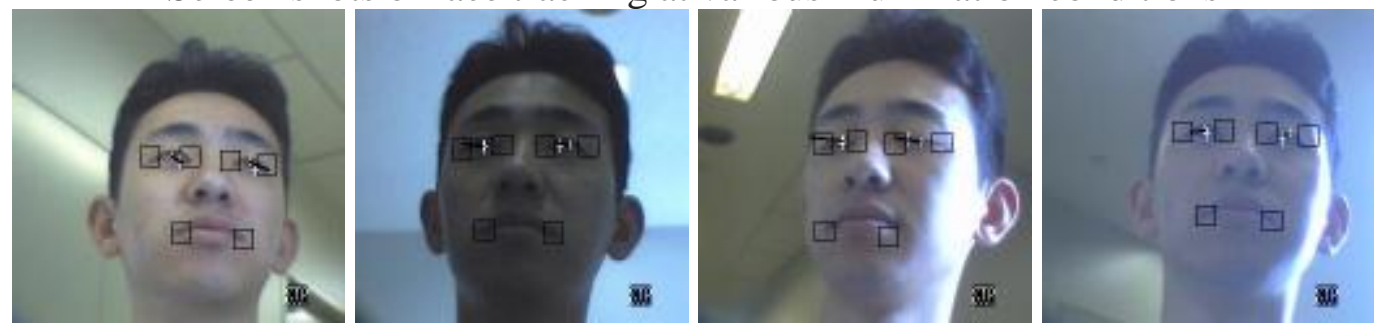

[Outdoor Experiment](2000/May/01)

Experimental Environment (a course around a pond in our institute)

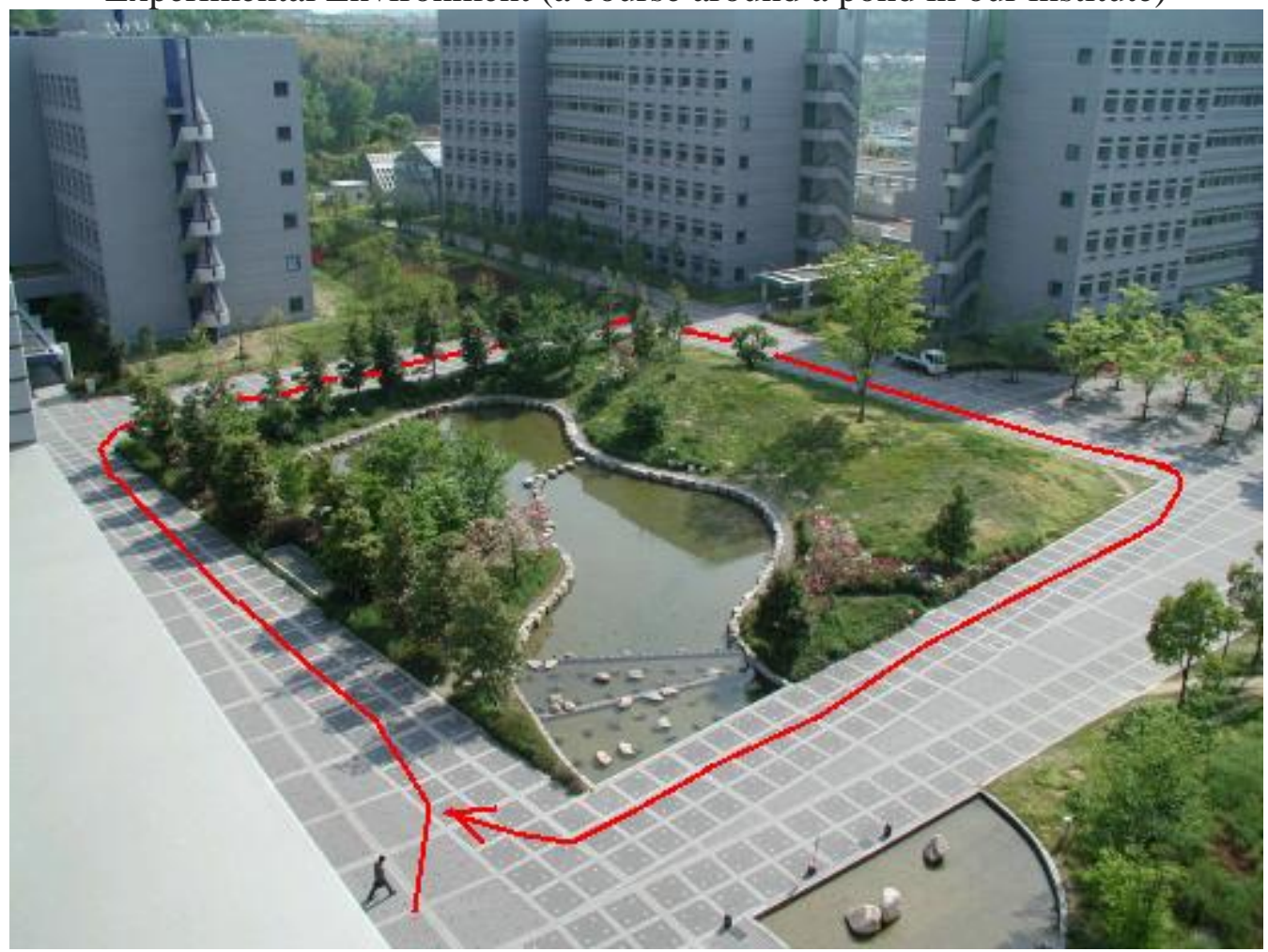

University of Essex - Intelligent RoboChair 
The main aim of this joint project is to explore and develop the advanced technology needed for a high performance and low-cost RoboChair which enables the elderly and disabled to gain necessary mobility to live independently and improve their quality of life in the society. This RoboChair should have a user-friendly man-machine interface and the ability of avoiding collision and planning a path. It will be equipped with a new vision system and a wireless communication system so that its carer or relative can monitor and tele-operate it when necessary. This project is jointed funded by the Royal Society and the Chinese Academy of Science, "Intelligent RoboChair: Improve Quality of Life for the Elderly and Disabled." The project is University of Essex and the Institute of Automation at Beijing plan to complete the project in 2007.

Professor Huosheng Hu will lead Essex’s Human-Centred Robotics team in developing algorithms for sensor fusion, map-building, intelligent decision-making, and tele-operation through the Internet using 3G mobile phones. Professor Kui Yuan of the Institute of Automation will develop prototype hardware and control software, including servo drivers, DSP-based control systems, sensor systems, and motion control algorithms.

[University of Essex, 2004]

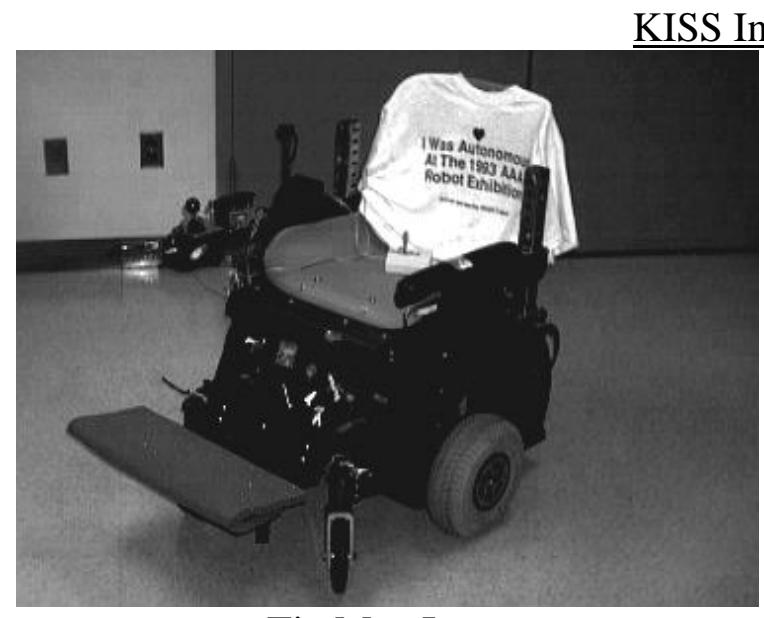

Tin Man I

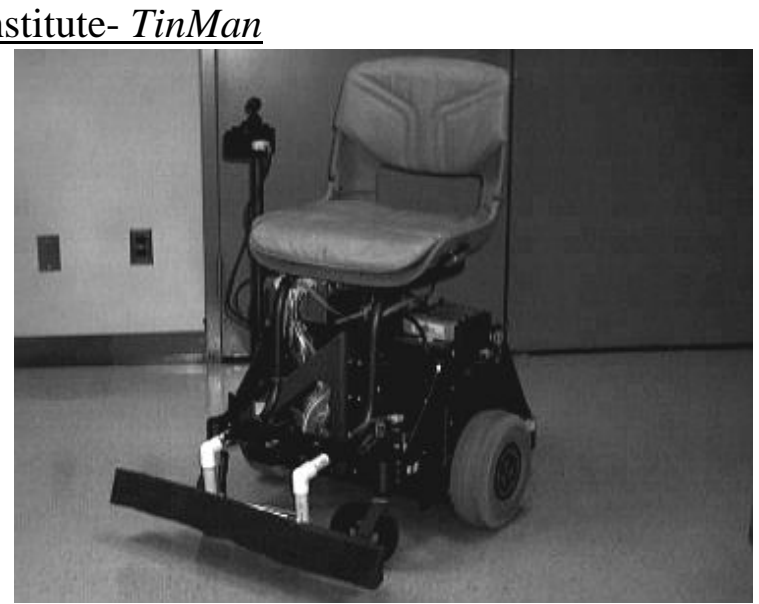

Tin Man II

For some people who are mobility impaired, the safe operation of a standard power wheelchair is beyond their capabilities. This is especially true for those individuals who do not have any fine motor control or who also have partial vision loss. The TinMan supplementary wheelchair controller is being designed to allow these individuals to be able to quickly, safely and independently operate their own wheelchair and easily direct their own movements through the world.

The TinMan project consists of a series of supplemental wheelchair controllers that have been developed over the past three years. These controllers sit between a power wheelchair's input device (e.g., joystick) and the standard wheelchair motor controller. The supplementary controller, along with its sensor network, allows the chair to sense many of the obstacles in its immediate surroundings along with doorways and other openings. The TinMan controller can then safely guide the chair past those obstacles and through the desired openings with a minimum of input from the chair user. 
The TinMan supplementary wheelchair controller is designed to be added to existing power wheelchairs. It has been tested with two of the industries most popular wheelchair control systems (The Dynamics and the Penny \& Giles series of wheelchair controllers). The retrofit takes only a few hours and should cost only a fraction of the cost of a standard power wheelchair. Any two-motor power wheelchair that uses proportional control should be able to accept a version of the TinMan supplemental controller. The controller will not work with scooters.

The TinMan controller has gone through several prototype versions. Several chairs equipped with the TinMan supplemental controller are in use at a variety of university research laboratories. It is the goal of KIPR to work with the industry to eventually make this technology available to all individuals who have need of it.

Some of the earlier versions of the TinMan system are discussed below.

Tin Man I was built by the KISS Institute and was its first foray into assistive robotics. Tin Man I is a robot wheelchair which can automate some of the navigation and steering operations which might prove difficult for someone with serious disabilities. In particular, this chair can find its way through doorways, follow hallways, and do limited amounts of dead reckoning navigation. One of the qualities that separates this wheelchair from other attempts at automated chairs is its cost. Only a few hundred dollars worth of equipment was added onto a stock power chair to create Tin Man I. [Miller,1995]

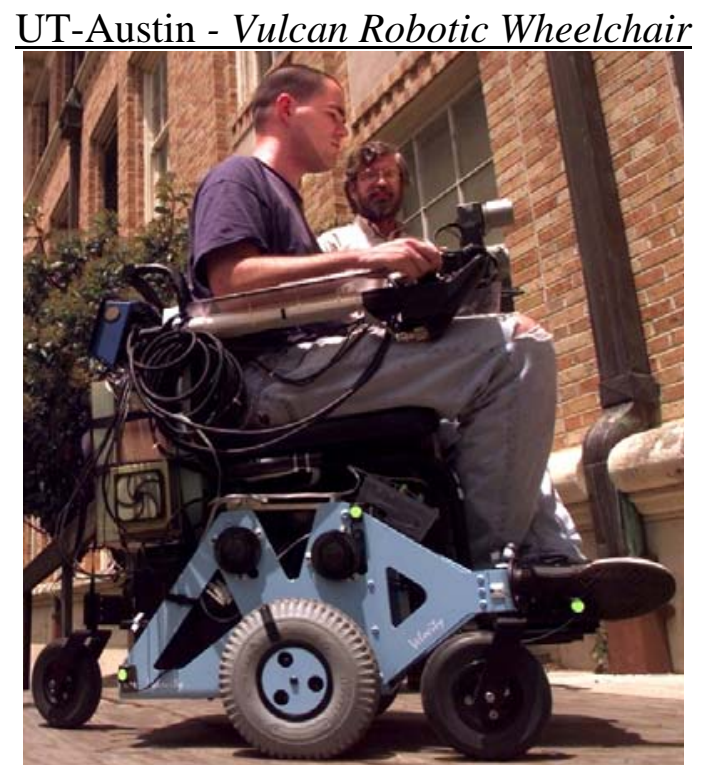

The Intelligent Wheelchair, Vulcan, is intended for people with normal cognitive and perceptual functioning and severe mobility and communication restrictions. This involves participation from several other groups in the Artificial Intelligence Lab in the UT-Austin Computer Science Department. There was an article written about the project in the Austin American-Statesman. [University of Austin, 1998]

\section{Seiki, Fujitsu - Tao Aicle}

These robots will safely transport passengers to their destination on automatic control while avoiding obstacles. The robot will be equipped with a computer and a sensor for autonomous 
movement and obstacle avoidance. An external infrastructure (i.e. server, wireless LAN, integrated circuit tag) can offer more accuracy and reliability of orbit control, remote monitoring of operating state, and safety information for users. [Seiki, Fujitsu, 2005]

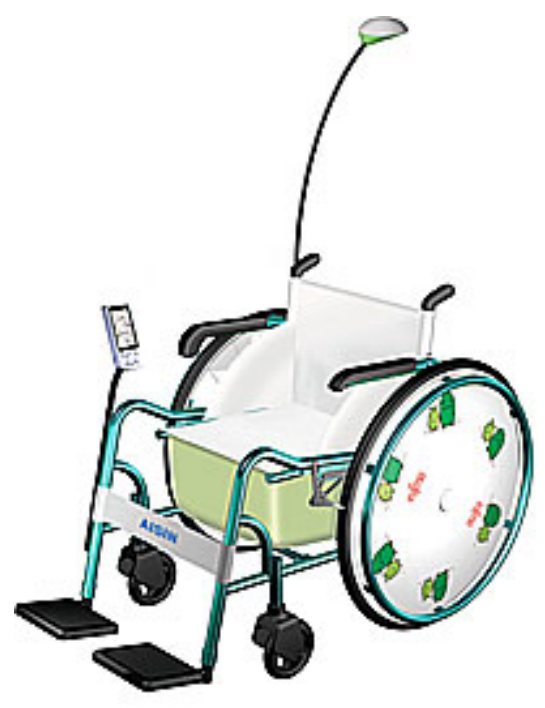

Application Illustration

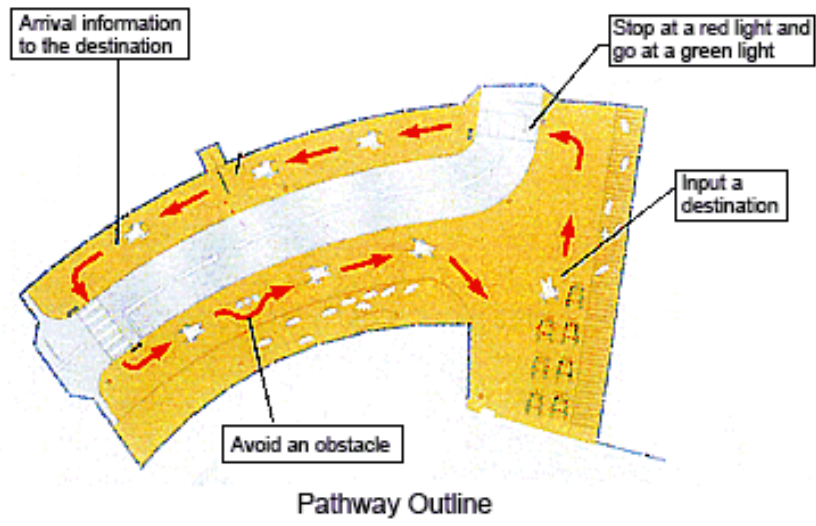

Toyota - Pod

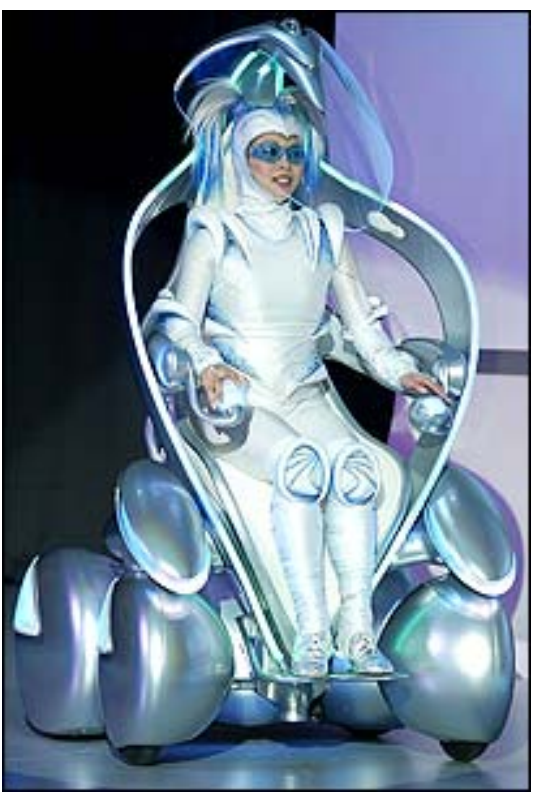

A new breed of wearable robotic vehicles that envelop drivers are being developed by Japanese car giant Toyota. The company's vision for the single passenger in the 21st Century involves the driver cruising by in a four-wheeled leaf-like device or strolling along encased in an egg-shaped cocoon that walks upright on two feet. Both these prototypes will be demonstrated, along with other concept vehicles and helper robots, at the Toyota stand at the Expo 2005 in Aichi, Japan, in March 2005.' [Toyota, 2005] 


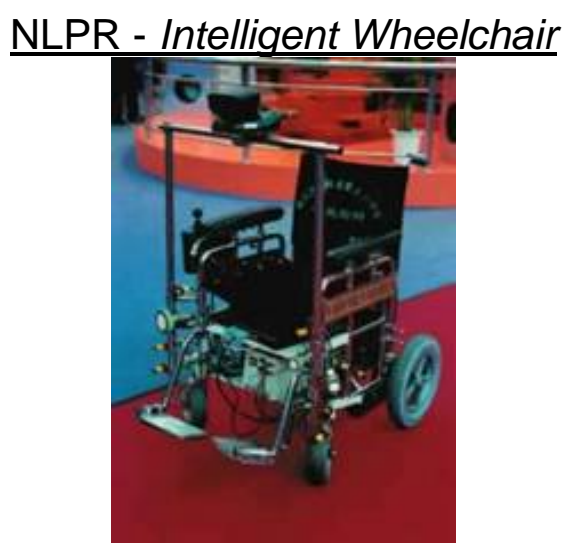

We do some experiments on the intelligent wheelchair of NLPR. The wheelchair is equipped with one CCD camera sensor, one audio input, 12 infrared sensors, 6 ultrasonic sensors and 2 DC motor drivers. It can perform some basic autonomous navigation tasks like obstacle avoidance, self-localization and can be controlled by some voice commands. It is a good platform to do the experiments of pose and gesture recognition.

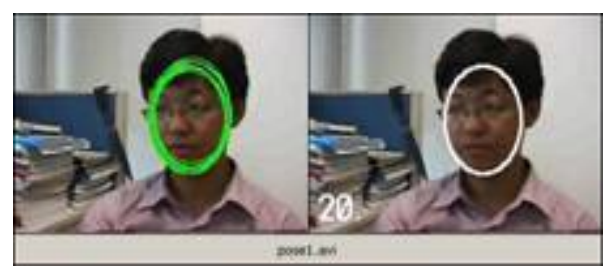

It is the module of head pose control mode, which is a part of Human-Robot Perceptual Interaction system on Intelligent Wheelchair. It aims to enable user to control the wheelchair just by his head pose, i.e. if you turn head right, wheelchair will turn right, and if you turn head left, wheelchair will turns left correspondingly . It is essentially a problem of real-time face tracking and head pose estimation. And how to detect the human face under variable illumination and changing background due to the movement of wheelchair is a big challenge. Here we provide a robust algorithm to detect human face and estimate head posture under dynamic moving environment. 


\section{University of Málaga - SENA Robotic Wheelchair}
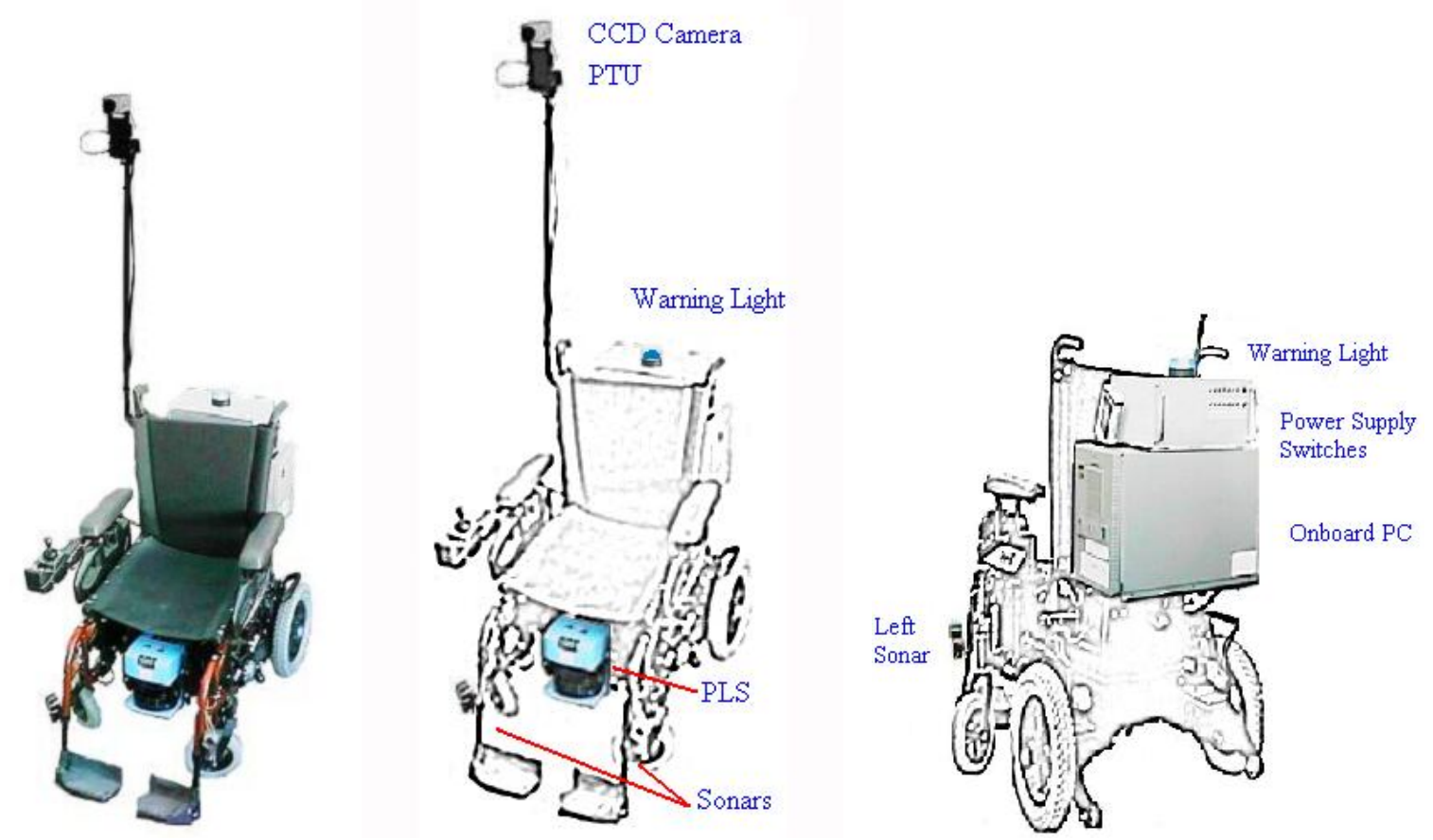

The SENA wheelchair, a mobile robot developed in the System Engineering and Automation Department of the University of Málaga. It is based on a commercial electric wheelchair that has been equipped with different sensors and actuators, managed from an onboard computer that is connected to remote stations via radio ethernet. The main goal of SENA is to serve for facilitating physical displacements to impaired persons.

Sensors that are available in the wheelchair include a CCD monochrome camera mounted upon a pan-tilt unit, a retractile $180^{\circ}$ radial laser scanner in the front of the chair, two radial scanning ultrasonic sensors, and several infrared proximity sensors.

The software architecture implemented for the SENA wheelchair has been built upon a system for integrating distributed software modules, developed in the last years, called BABEL. Under this system, a set of software modules can communicate and coordinate in order to make up a complete robotic application, possibly distributed among a number of computer connected by a network.

\section{Wheelchair Standards}

Why Bother with Standards? [Hobson, 1999] Hobson wrote these answers to his question:

- Standards ensure minimum levels of performance.

- Allows accurate comparison between products.

- Stimulates quality improvement.

- Reduces manufacturer's liability exposure.

The American National Standards Institute (ANSI) began the thrust for voluntary wheelchair standards in 1979 with 18 (now 23) standard procedures for testing and comparing wheelchairs. The facilitator for this effort is the University of Pennsylvania. Standards are administered 
through RESNA (Rehabilitation Engineering and Assistive Technology Society of North America) where: their procedures ensure minimum wheelchair performance and safety levels and they provide a basis for consumers and clinicians to compare products during wheelchair selection. The standard includes both the manual and powered wheelchairs along with seating standards. Presently, there don't appear to be any intelligent (robotic) wheelchair standards nor any lift-wheelchair standards.

Rory Cooper, Director, Human Engineering Research Laboratories (HERL) stated that:

- "There is no pending RESNA or ISO work item for intelligent wheelchairs. RESNA and ISO typically don't develop Assistive Technology standards until there are multiple manufacturers producing and selling product.

- There are Society of Automotive Engineering standards for lifts, but again not for anything intelligent.

- Any standards work item requires funding to support the research and validation of the test methods. An agency or foundation would need to support the work.” [Cooper, 2005]

ANSI/RESNA Wheelchair Standards

Voluntary Industry Standards

- USA/Canada/International Standards Organizations collaboration

- ANSI/RESNA standards-US

- Work began, 1979---now 23 parts in total.

- Type: mainly information/performance disclosure--some pass/fail.

- Test methods to verify performance/safety.

- Required information disclosure in presale literature.

Volume One contains standards that apply to both manual and electric wheelchairs and scooters. Volume Two contains additional standards that apply to electric wheelchairs and scooters. Standards are sold in volumes only. Section 19-Wheelchairs used as seats in motor vehicles has recently been approved.

ANSI/RESNA Wheelchairs Standards Volume 1 and 2 consist of the following sections: Volume 1 contains standards for manual and electric wheelchairs and scooters.

Volume 1: Requirements and Test Methods for Wheelchairs (including Scooters)

- Section 0 - Nomenclature, terms, and definitions

- Section 1 - Determination of static stability

- Section 5 - Determination of overall dimensions, mass, and turning space

- Section 7 - Determination of seating and wheel dimensions

- Section 8 - Static, impact, and fatigue strengths

- Section 11 - Test dummies

- $\quad$ Section 13 - Determination of coefficient of friction of test surfaces

- Section 15 - Requirements for information disclosures, documentation, and labeling

- Section 16 - Determination of flammability

- Section 19 - Wheelchairs used as seats in motor vehicles

- Section 20 - Determination of the performance of stand-up wheelchairs

- $\quad$ Section 22 - Set up procedures 
- $\quad$ Section 93 - Maximum overall dimensions

Volume 2 contains additional standards for electric wheelchairs and scooters only. Volume 2: Additional Requirements for Wheelchairs (including Scooters) with Electrical Systems

- Section 2 - Determination of dynamic stability of electric wheelchairs

- Section 3 - Determination of efficiency of brakes

- Section 4 - Determination of energy consumption of electric wheelchairs

- Section 6 - Determination of maximum speed, acceleration, and retardation of electric wheelchairs

- Section 9 - Climatic tests for electric wheelchairs

- Section 10 - Determination of obstacle-climbing ability for electric wheelchairs

- Section 14 - Testing of power and control systems for electric wheelchairs

- Section 21 - Requirements and test methods for electromagnetic compatibility of powered wheelchairs and motorized scooters

Wheelchair Standards Efforts:

- ANSI-RESNA Wheelchair Standards

- ANSI/RESNA/SAE Transportation Safety Standards

- International Standards Organization (ISO) W/C Transportation Standards

Regarding testing of power and control of powered chairs, [Hobson, 1999] points out that test information is pass/fail and the standards specify results of 17 tests related to the safety of the electrical power and control systems. Also, he states that serial interface for electric wheelchairs controllers specify electronic interface for connecting a motor controller to a joystick and other accessories (ECUs, computers, AAC devices) and that there are some software communication protocols. There is currently no standards requirement or procedure as a safety precaution to the rider for testing the cognizant ability of the rider to control the powered chair. A powered chair rider can therefore, be full-throttling the acceleration while looking another direction causing a safety hazard to him/herself or others. Hobson says the electronics part of the standard "was intended to establish an electronic standard for the method by which wheelchair controls would communicate with other electronic equipment in the user's environment, such as computers, communication devices or environment control devices. Unfortunately, it did not proceed quickly enough through the required stages of development and was terminated by ISO.”

With regard to stair climbing devices that could perhaps lead to lift type devices for wheelchairs, [Hobson, 1999] says the standards specify:

- requirements and test methods for stair climbing devices (wheelchairs and wheelchair carriers),

- ergonomic considerations for user and attendants, and

- labeling and other information disclosure requirements

Moreover, a part of Hobson's document briefly discusses the suggestion to add a determination of performance of stand-up wheelchairs that more closely deals with lift type mechanisms that could accompany or be a part of wheelchairs. The suggested standard would specify modifications to a dummy test series to allow for standing and would specify displacement 
between the user and wheelchair during transition from sitting to standing. In 1999, this effort was at the voting stage through ISO with no update at the time of this report.

Wheelchair Testing Laboratory

University of Pittsburgh - HERL Laboratories [HERL]

Assistive Technology Evaluation Laboratory

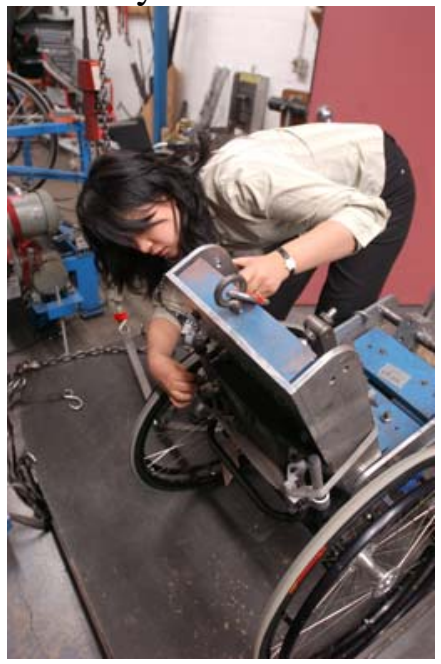

The Assistive Technology Evaluation Laboratory is used to develop standards for assistive technology, and to test assistive devices for compliance with existing standards. This laboratory contains a full compliment of testing equipment for wheelchair standards, and limited equipment for other types of assistive devices. Major equipment includes double-drum testers, curbdrop testers, a treadmill, static testing machine, and an environmental chamber. This is one of the most comprehensive laboratories in the United States equipped to provide testing of manual and power wheelchairs to ANSI/RESNA and ISO wheelchair standards. Laboratory personnel are actively involved in developing wheelchair standards. We currently have critical roles in the development of several national and international standards. This laboratory also provides testing and design services to industry, consumer groups, insurance agencies, and government agencies.

Activities of Daily Living Laboratory

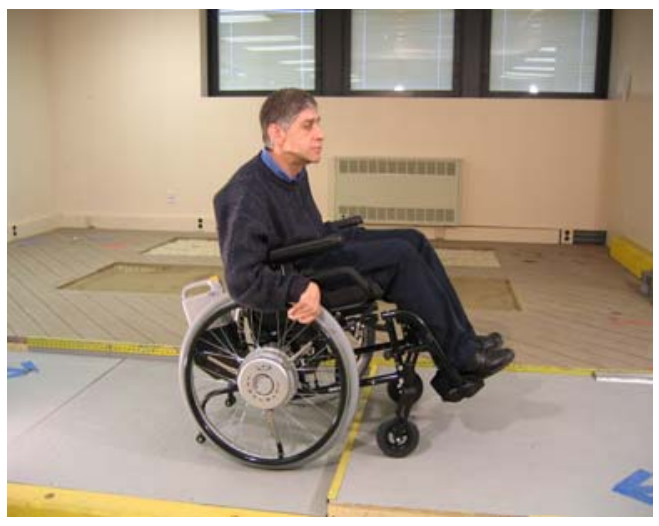


The Activities of Daily Living (ADL) laboratory establishes an environment for the realistic simulation of everyday objects (desks and counters), situations (grocery shopping and banking), and obstacles (ramps, stairs, and gravel) which are encountered by individuals using mobility aids. This simulated environment allows for a controlled study environment to test ADL skills and routine tasks of wheelchair users in their own or new mobility devices.

Robotics Laboratory

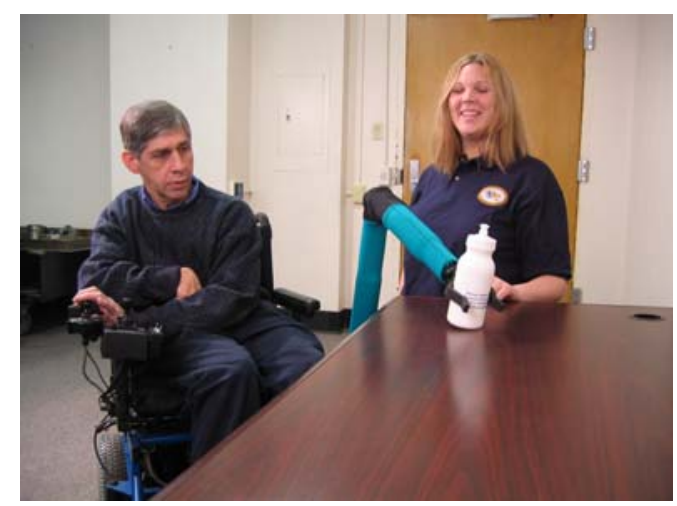

Advances in engineering technology have resulted in the development of robotic devices that enhance mobility and functional independence as well as prevent secondary complications of physical impairments. In the Robotics Laboratory, studies are being conducted to evaluate the design and performance aspects of robotic technology with an overall objective to improve the quality of life of individuals with mobility deficits. Engineers and clinicians from HERL work closely with robot developers and manufacturers to provide devices that safe, reliable, functional, and practical and that will allow greater interaction and control of the physical environment.

Whirlwind Wheelchair International

Perhaps one problem with wheelchair dependents is simply not having the equipment. Whirlwind Wheelchair International works to make it possible for every person in the developing world who needs a wheelchair to obtain one that will lead to maximum personal independence and integration into society. In order to fulfill this mission, WWI seeks to give wheelchair riders a central role in all of its projects and activities.

Whirlwind Wheelchair International (WWI) is a program of the Urban Institute at San Francisco State University (SFSU). Founded in 1989 as the Wheeled Mobility Center by SFSU Engineering professor Peter Pfaelzer and paraplegic engineer and wheelchair designer Ralf Hotchkiss, WWI grew out of Hotchkiss's work since 1980, traveling the globe designing wheelchairs that could be built in developing countries from locally available materials. 
WWI technology has been taken to 45 countries. Hotchkiss's pioneering work has led to many innovations that are integral to wheelchair models produced by many workshops and NGOs around the world. All of WWI's designs are placed in the public domain in order not to add to the cost of village wheelchair shops using these designs.

From its inception, WWI mostly concentrated its efforts on establishing small shop production in Africa, Asia, and Latin America. In 1998, WWI formed a partnership with Physicians Against Land Mines (now Center for International Rehabilitation) in Chicago and later developed a plan to get wheelchairs to Afghanistan. Parts for the newest Whirlwind, the Ultra-Liviano, are now being made at the Worth Trust factory in Vellore, India, to be shipped to Afghanistan for assembly. WWI will train disabled Afghans to assemble and fit the chairs to individual users.

In early 2003, WWI formed a strategic consortium with the Arthur B. Schultz Foundation in the U.S., the HandiNor wheelchair factory in Fetsund, Norway, and the Atlas Alliance in Norway for higher volume production of high-quality Whirlwind-style wheelchairs in developing countries in Africa, Asia, and Latin America.

The consortium, known as the Whirlwind Industrialization Project (WIP), starts with Whirlwindgenerated designs based on years of experience as well as on continuous feedback from wheelchair riders in the field, called the Whirlwind Network. Whirlwind designers then work with HandiNor's engineers to retain the important design characteristics of the Whirlwind wheelchair while making it easier and cheaper to produce in large volumes in small to medium size shops with a capacity of up to 300 wheelchairs per month. HandiNor also uses its expertise in manufacturing wheelchairs for the European market to design and produce advanced jigs and fixtures that the project supplies to the local shops. Whirlwind provides on-site training in wheelchair assembly, production, and fitting. The Arthur B. Schultz Foundation funds startup costs, and along with the Atlas Alliance provides funds to support local wheelchair markets.

WWI is committed to the development of wheelchair standards appropriate to local conditions and to the adoption of such standards by the governments of developing nations. To further that effort, WWI now attempts to build a Standards and Testing component into each of its projects.

WWI offers consulting services to private wheelchair manufacturers and individual designers and inventors. Each semester, WWI gives a hands-on wheelchair design and construction class at San Francisco State University to students and interested members of the community.

[Whirlwind Institute, 2005]

\section{Assisted Lift Chairs}

\section{LIFT CHAIRS}

$\underline{\text { AAT Alber Antriebstechnik GmbH - S-Max }}$ 

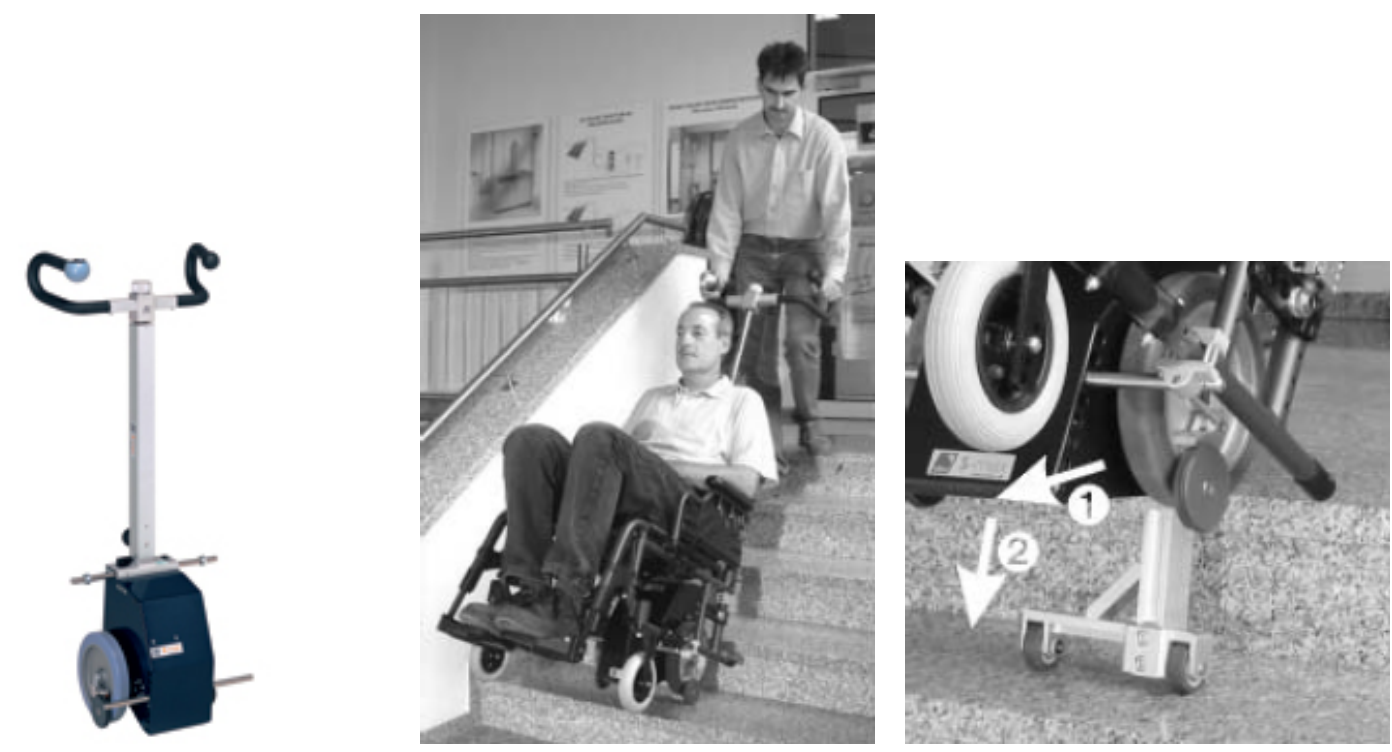

The S-Max is a powered lift device that, with the aid of another person, can lift the wheelchair dependent and chair up and down stairs and slopes. [AAT]

\section{(Unknown Manufacturer and Model)}
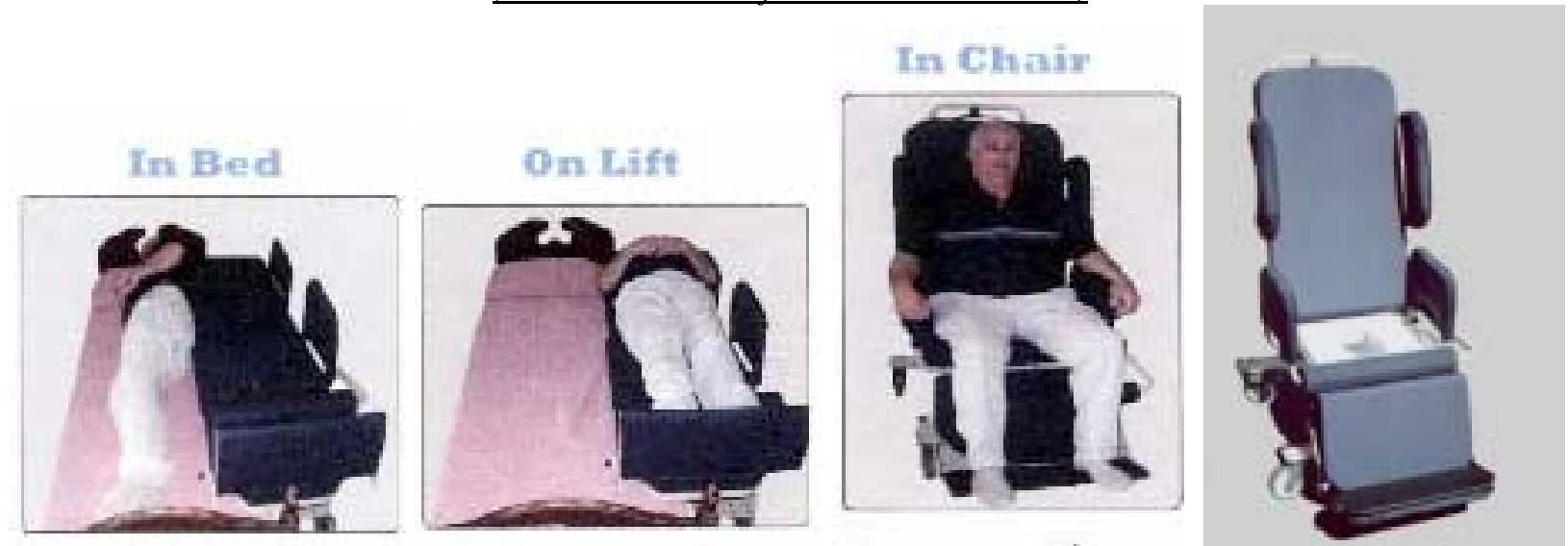

Also, specialized toilet chair is shown on the right. This chair is an assistive patient cart to be mobilized by a caregiver. 
Draft: 9/15/06

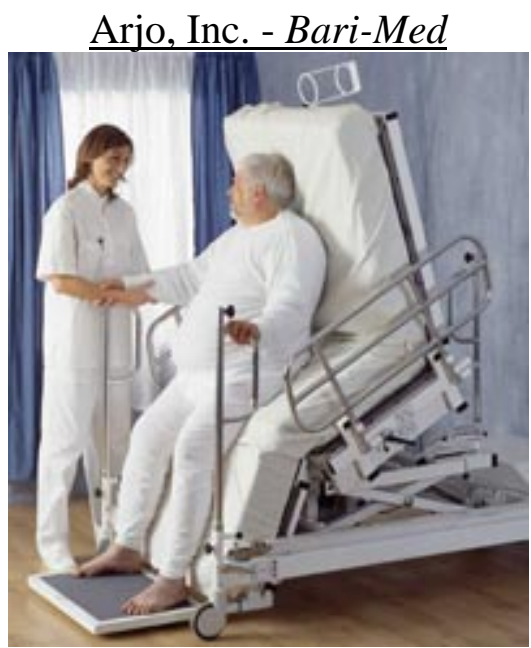

The Bari-Med from Pegasus has been designed to especially address the needs of caring for the obese patient, combining comfort, security and dignity. The Bari-Med is ideal for the larger patient whose body weight and/or size exceeds that of a normal bed frame and it will improve the moving and handling of the larger patient to ensure ease of care and safer working conditions for the caregiver. [Arjo-Bari-Med, 2005]

\section{Non-Assisted Lift Chairs}

Johnson \& Johnson - Independence 3000 IBOT Transporter
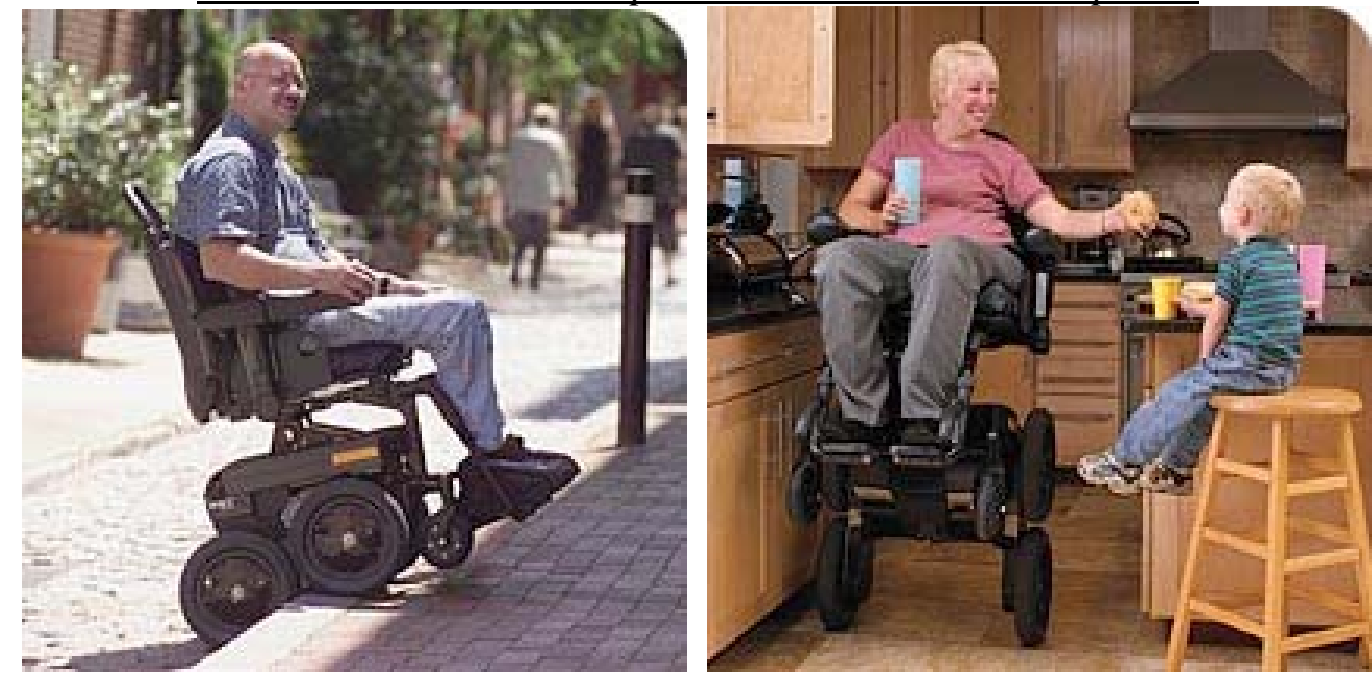
Draft: 9/15/06

Bounder Wheelchairs - Scissorlift Wheelchair

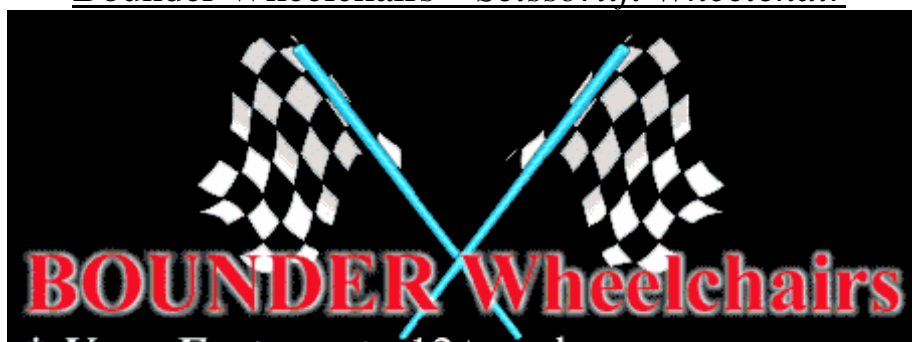

* Very Fast - up to 12+ mph

* Extrem ble

* Baria 1000 lb users

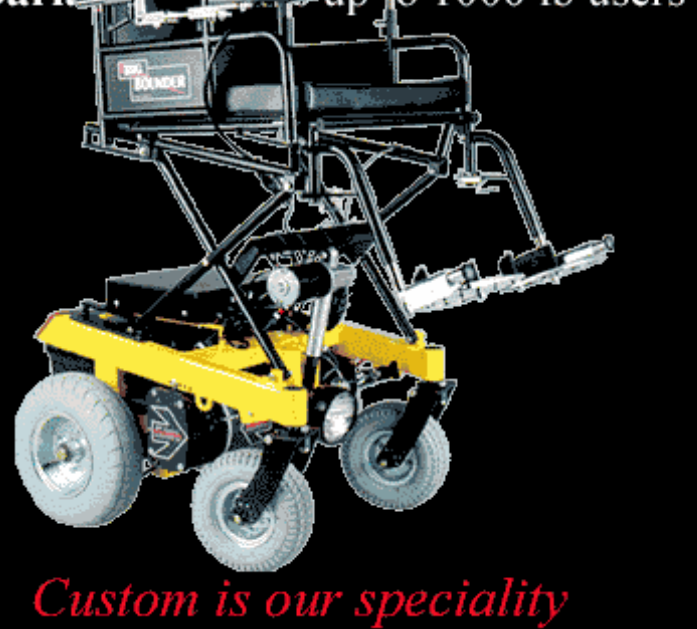

$\underline{\text { PerMobil - Chairman } 2 K \text { Vertical and Corpus }}$
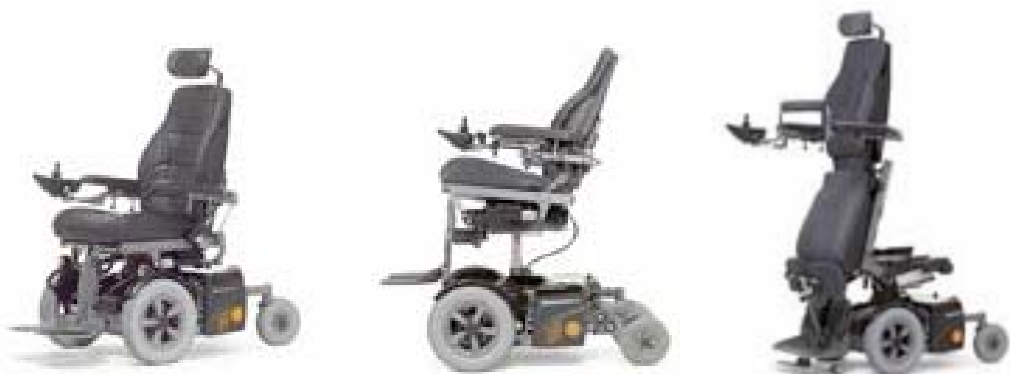

PerMobil is a Swedish powered wheelchair manufacturer with branch offices in many countries, including the US. 


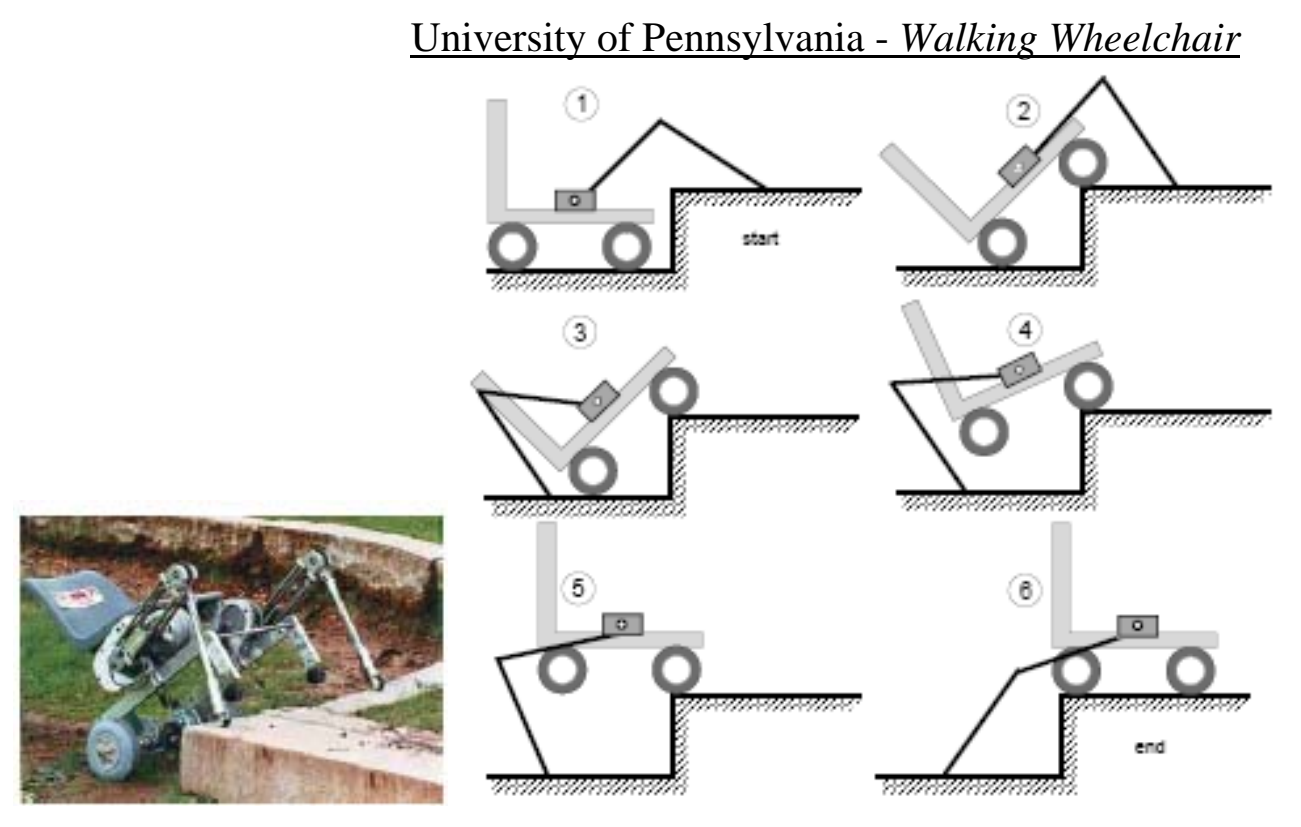

[University of Pennsylvania, 1994]

\section{NIST - Phase 1 HLPR Chair (formerly RoboChair)}

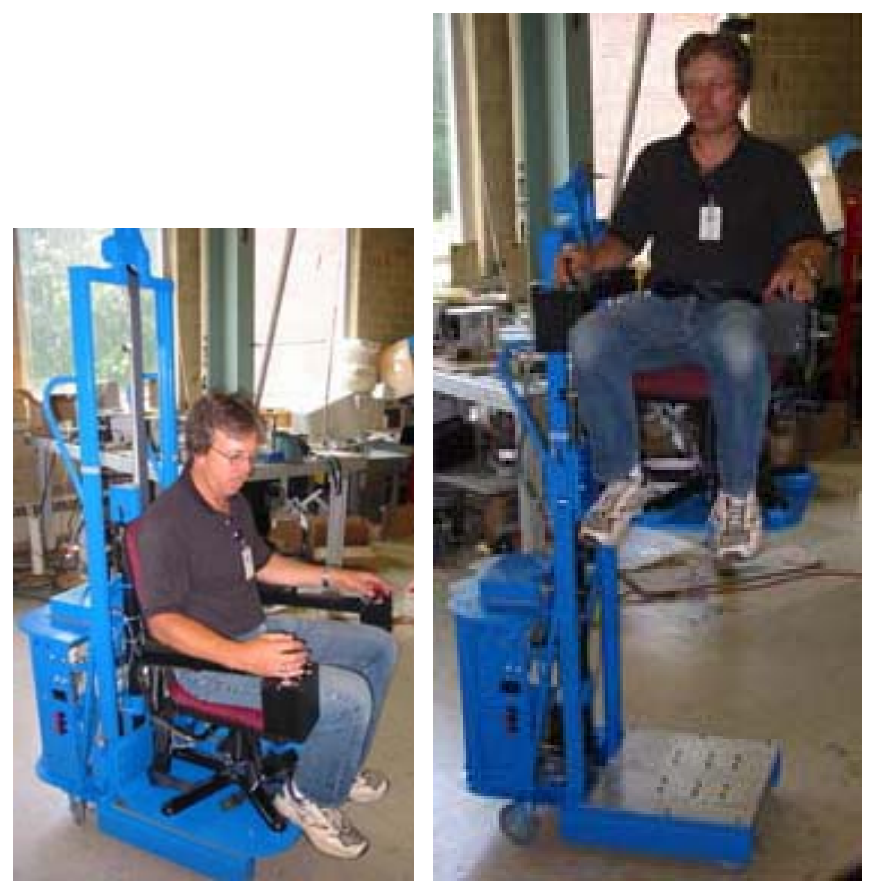

RoboChair 1 demonstrated patient mobility with skid-steer control and lift of approximately $1 \mathrm{~m}$ high while seated in the chair attached to the lift structure. 


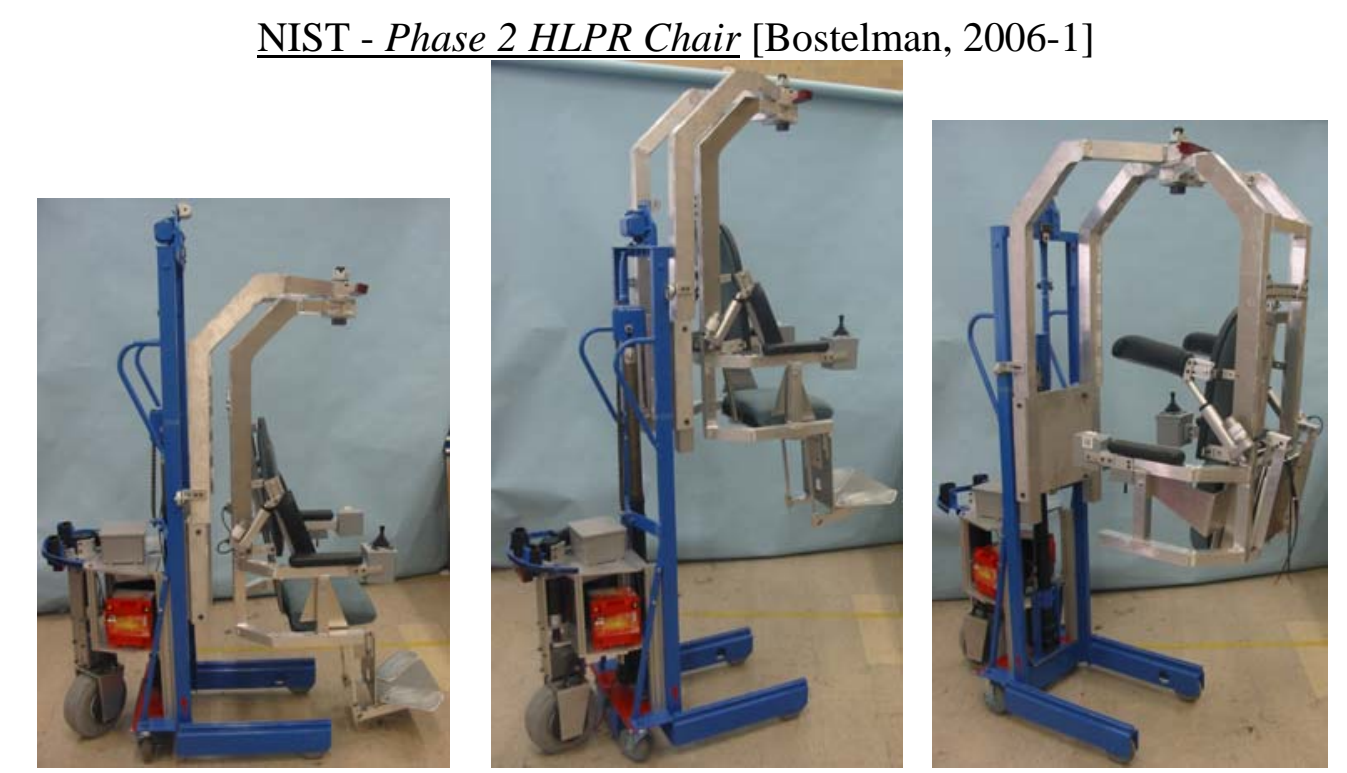

Left to right: mobility, lift and patient place-in-chair configurations, respectively.

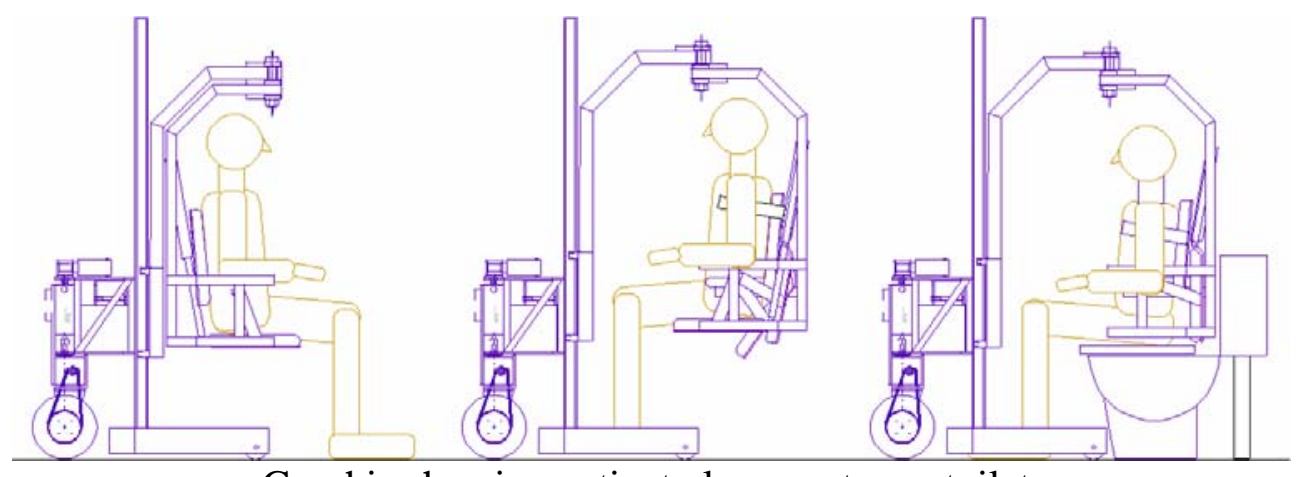

Graphic showing patient placement on a toilet.

HLPR Chair 2 was designed for: patient mobility; placing patients on seats, chairs, bed, etc.; lifting the patient to approximately $1 \mathrm{~m}$ above the mobility position; and rehabilitation (see Rehabilitation Section). Therefore, the HLPR Chair is designed to, independently or with caregiver help: provide flexibility for the patient to reach tall objects (e.g., load and unload the dishwasher); take the patient to the bathroom and support them during, place the patient on a bed; have a support structure to stand and walk with; and many other typical normal tasks. It is designed as a testbed for the healthcare industry to develop control algorithms for patient lift and mobility, as well as docking and patient placement control testing and to serve as a research step towards future patient lift devices for homes and medical facilities. [Bostelman 2006-1\} 


\section{Toyota - Car for Disabled Drivers}

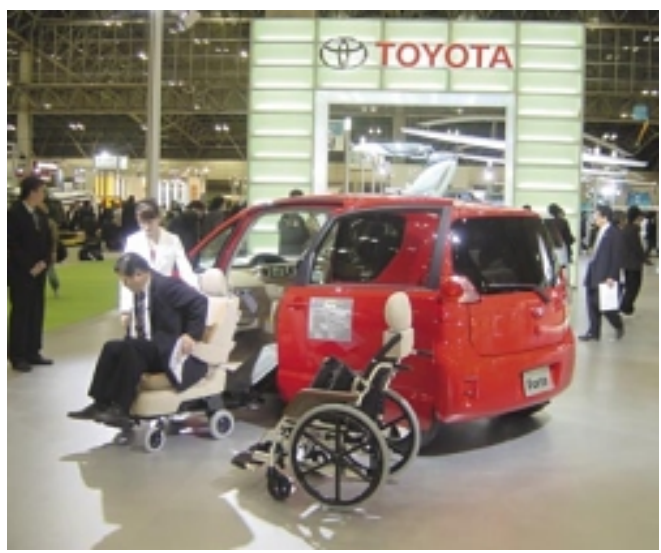

This year's concepts and production models hinted that Japan might soon break into the lead in automotive products for drivers with disabilities just as it has done in the U.S. with its passenger cars. And along with the new designs came an important message: Designing and marketing vehicles for disabled drivers could become an extremely important and lucrative segment of the automobile industry. [Thomas, 2005]

\section{Isuzu - Gigamax Tractor Ability Cab}

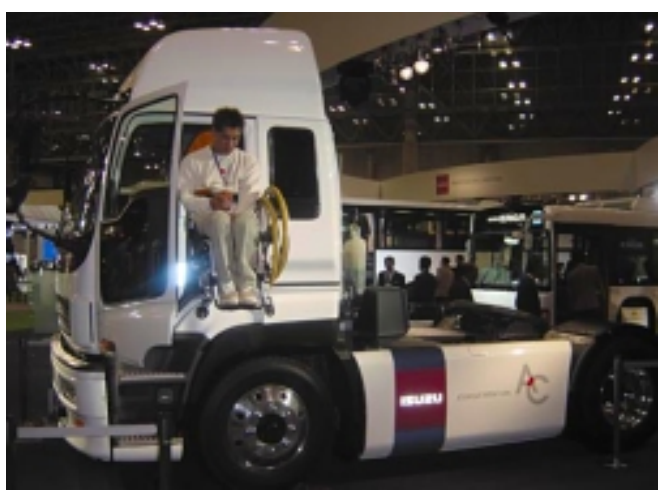

Most of us don't generally think of Isuzu as a manufacturer of vehicles for disabled drivers. Isuzu builds a full range of trucks and buses, including accessible transit buses that are in use throughout Asia. But they also make one of the most innovative vehicles for the disability market--the Isuzu Gigamax Tractor Ability Cab--a 7,500-pound long-haul big-rig tractor that is factory built to be driven by a paraplegic. 


\section{Waseda University - Bipedal Robot}

New bipedal robot can climb stairs carrying people. Robot developer Tmsuk Co. and a Waseda University research team have created a bipedal robot that can ascend and descend stairs while carrying a person. The developers modified an earlier version intended for flat surfaces. They aim to commercialize the WL-16RII robot by 2010, hoping it will be used to provide greater mobility to physically handicapped people. The Kitakyushu-based firm's earlier models were not able to go up and down stairs due to the limited range of motion of the legs. The developers made stair climbing possible by enabling the robot to twist its hip when it steps. "This is the first two-footed robot in the world that can go up and down stairs while carrying a person," said Atsuo Takanishi, a professor who heads the Waseda University team. The robot also has an onboard computer that

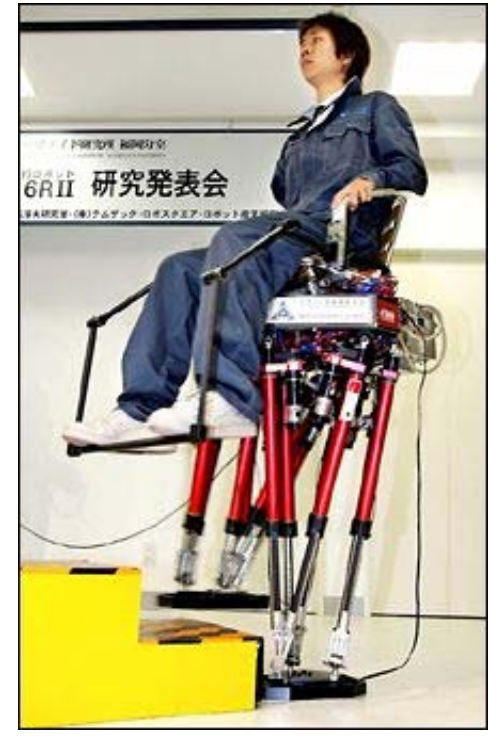
senses differences in elevation. It employs proprietary technology in which six actuators support the legs moving together. In tests so far, the robot handled stairs with steps $20 \mathrm{~cm}$ high while carrying a person who weighed $94 \mathrm{~kg}$.

\section{Duke University - Lifting Assist}

Our client is a seventeen-year-old girl with cerebral palsy. She cannot get into her motorized wheelchair comfortably without the assistance of others. The goal of this project was to build a device that will allow her to ascend into her wheelchair as independently as possible. We achieved this goal by constructing a lifting assist that is used in conjunction with her power wheelchair, which has an 8" extending actuator. The device lifts her 8" off the ground to make sitting in the wheelchair easier; it also serves as a footrest at all times during use of the wheelchair. This novel device will give our client new independence, and minimize the help required from others. [Duke University, 2004]

\section{REHABILITATION DEVICES}

Ken Paulson, 44, suffered a spinal cord injury in 1997 resulting in near-paralysis of his leg muscles and an inability to step or walk in a natural way. During "partial weight bearing” physical therapy research sessions a few times per week beginning 2000, led by Dr. Herman and Dr. He at the Rehabilitation Institute at Good Samaritan Regional Medical Center (GSRMC) in Phoenix, Ariz., Ken can walk up to 15 m (50 ft). [Banner, 2001] Paulson still needs a walker but no longer requires the physical support of therapists to move his partially paralyzed legs. In his walks he moves his legs in a coordinated pattern that is relatively smooth, rhythmic and consistent. Additionally, his walking requires much less effort. It has been demonstrated by medical persons that even an injured person who was deemed quadriplegic could walk again by first providing physical therapy to exercise the leg muscles. Once PWB facilitates a more normal stepping pattern (the individual is not yet able to achieve functional walking), an electrical stimulator is surgically implanted in the spinal cord on a site below the level of the SCI. Ken's 
story is encouraging for wheelchair dependents and for the development of leg exercise equipment that is designed to allow the patient to be partial weight bearing.

The following off-the-shelf rehabilitation devices, for use mainly by wheelchair dependents, are generally exercise equipment (e.g., hand cycles, universal weight machines, gliders) developed to stimulate muscle activity while seated. An exception is the Altimate Medical Easystand which provides muscle exercise from a standing position for those that can, even dependently, stand. For independent use of these equipment by the wheelchair dependent, initial training by a caregiver on the use of the equipment, as well as enough cognitive ability by the wheelchair dependent to use the equipment independently would be required.

\section{Saratoga - Hand/Arm Exercisers}
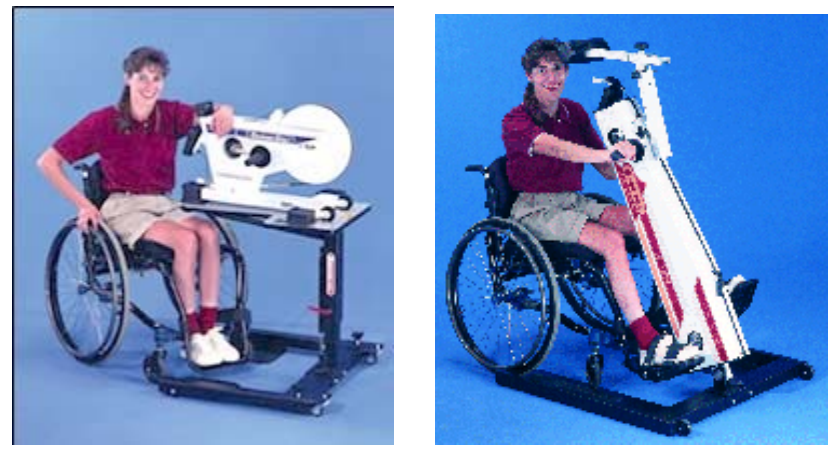

The Colorado Sport Cycle ${ }^{\mathrm{TM}}$ (left photo) incorporates many features of the Saratoga Cycle ${ }^{\circledR}$ (right photo) - such as its main frame and flywheel identical in quality and rugged durability - but is a lower-priced, more basic design for persons who do not need the extensive features of the Saratoga Cycle ${ }^{\circledR}$. [Saratogo, 2005]

\section{Uppertone - Upper Body Exerciser}

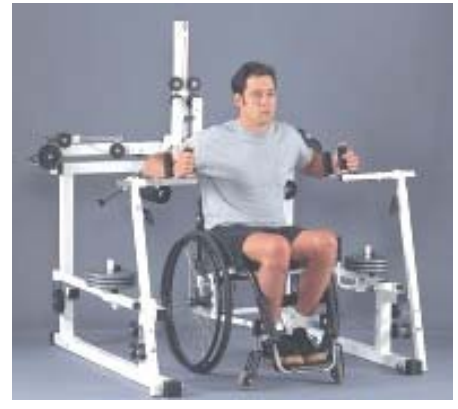

The only product of its kind, the Uppertone was designed and introduced in 1990 by a C4-C5 quadriplegic. It allows people with $\mathrm{C} 4-\mathrm{C} 5$ and below quadriplegia (tetraplegia) due to spinal cord injury to do all the necessary upper body exercises necessary for rehabilitation and maintenance, without any assistance. Indeed they can make all the adjustments, including resistance, without handgrip strength, cuffs, or assistance. The Uppertone is now in use in spinal cord injury treatment centers, rehabilitation facilities, universities and colleges, and homes in more than twenty countries. [Uppertone] 


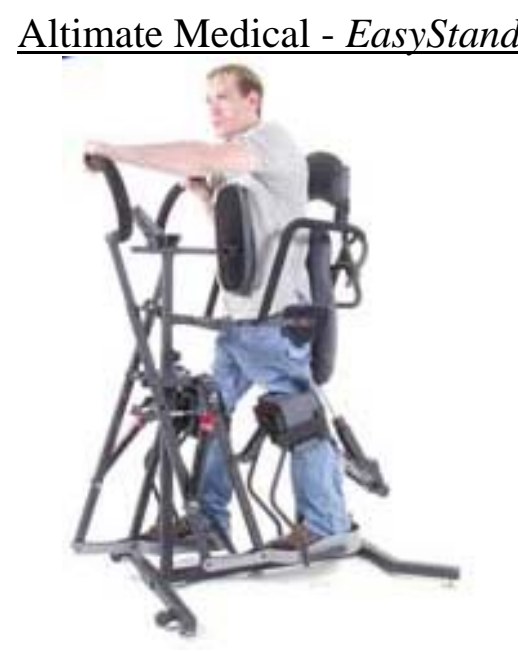

The EasyStand 6000 Glider is an active stander that provides reciprocal movement of the arms and legs while standing. It provides active range of motion of hips, knees, and ankles while strengthening the upper extremities. [Altimate Medical] The EasyStand is typically used: when hips and knees tighten up due to wheelchair use, to increase the heart rate and strengthen the cardiovascular system, and/or immediately after injury for the greatest benefits.

\section{University of Delaware - Leg Anti-Gravity Device}
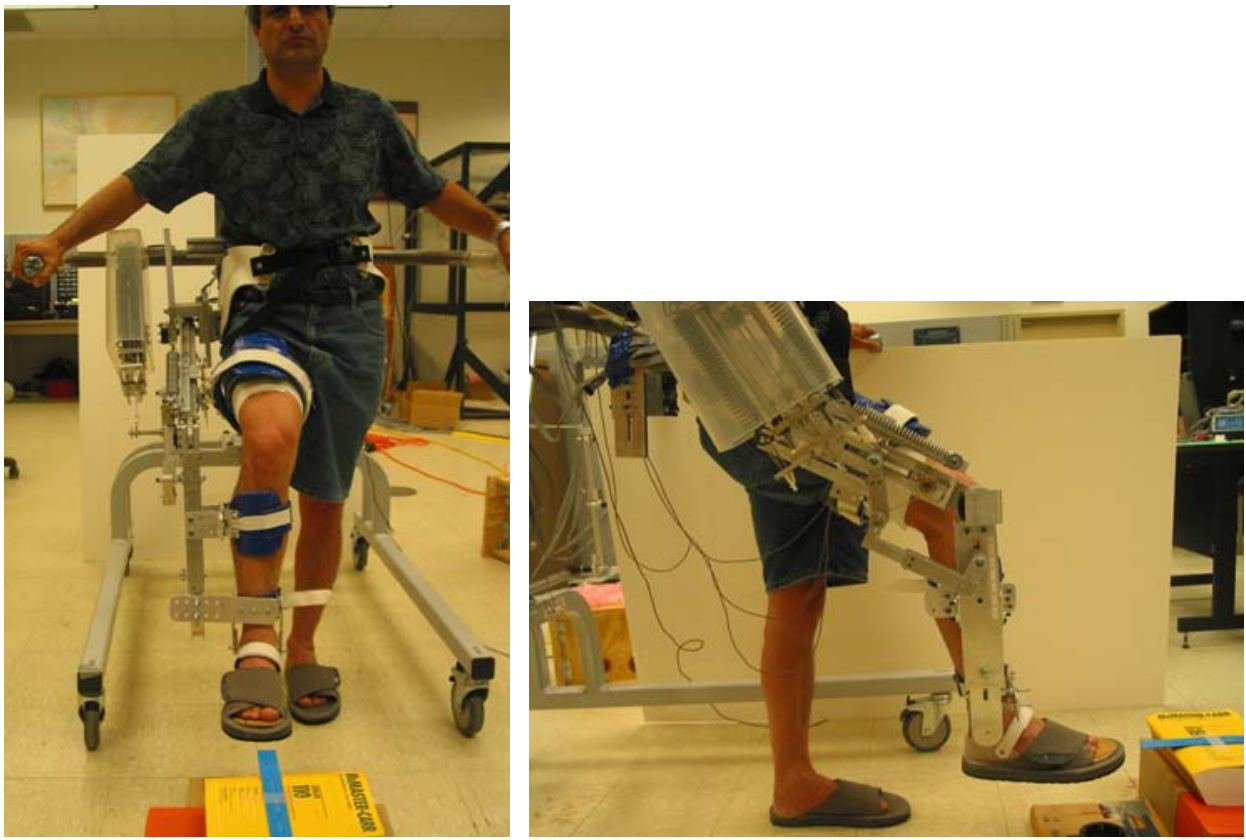

University of Delaware designed and built a leg anti-gravity device to minimize the leg-lift requirement for stroke victims to relearn to walk. It uses mechanical linkage and springs to support leg loading. [University of Delaware, 2005] 


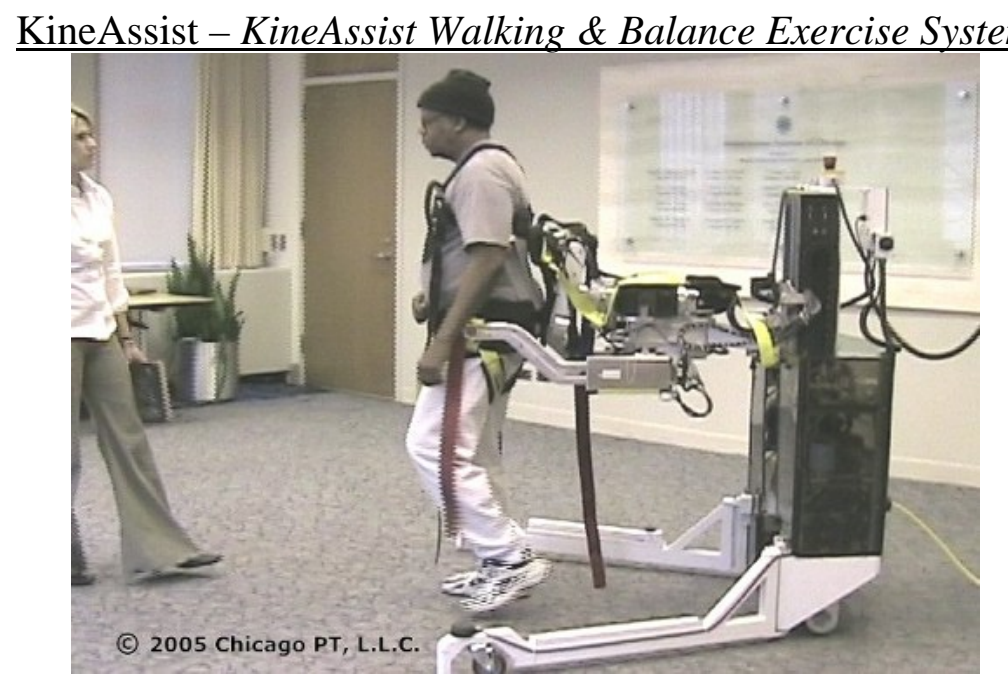

KineAssist ${ }^{\mathrm{TM}}$ is a physical therapist's tool, not a machine that provides physical therapy. KineAssist ${ }^{\mathrm{TM}}$ maintains the therapist's connection with the patient in all its important aspects: physical, sensory and psychological. The KineAssist ${ }^{\mathrm{TM}}$ device is indicated for use by physical and/or occupational therapists in a rehabilitation setting for assisting/enabling therapeutic exercises, neuromuscular re-education and gait and balance training. This device was sponsored by a NIST Advanced Technology Program grant and by the Rehabilitation Institute of Chicago in June 2003. Chicago PT, LLC was formed by a team of clinical and research physical therapists, engineers, and faculty of Northwestern University.

[ChicagoPT, 2005]

\section{NIST - Phase 2 HLPR Chair applied to Rehabilitation}
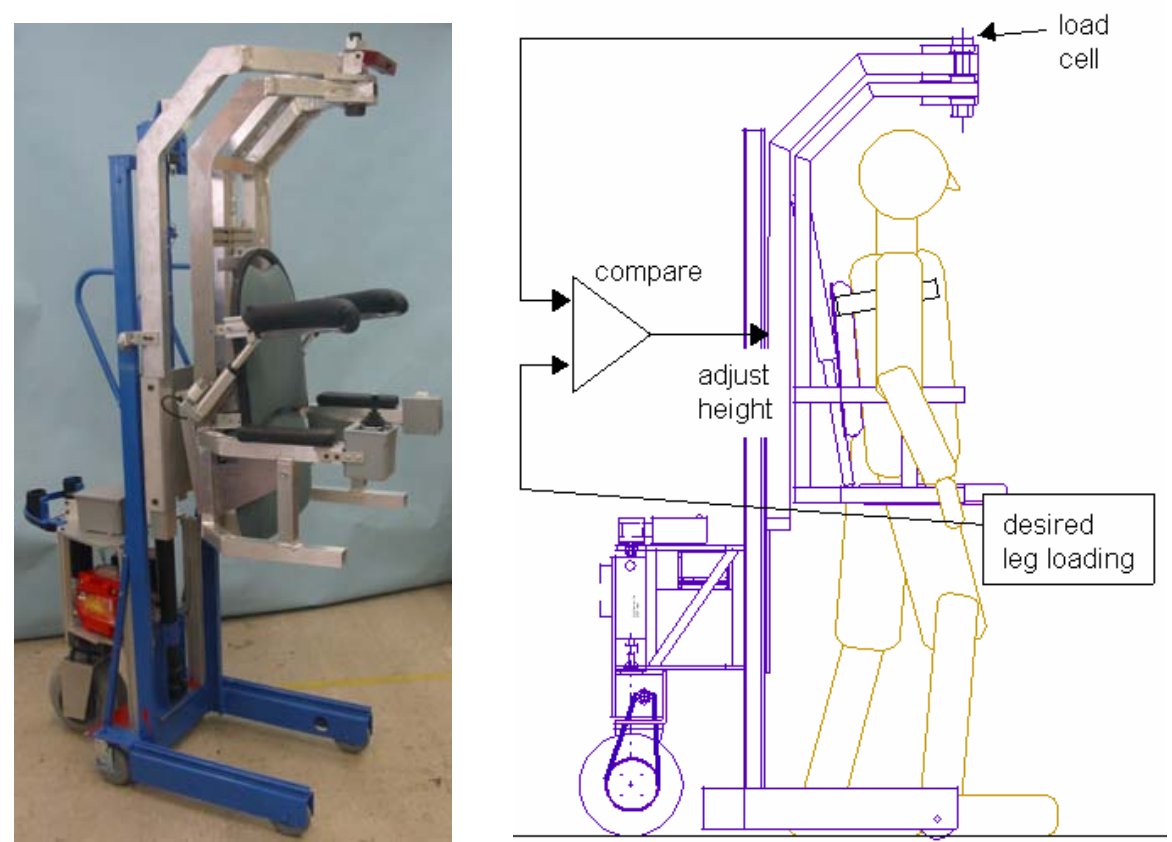

The HLPR Chair includes footrest fold and seat rotate behind the patient while he/she is lifted with torso lifts. The patient lift continues to move up lifting the patient as they move their legs beneath them to standing position. The HLPR Chair frame includes an open U-frame base to 
allow feet access to the floor directly beneath the patient so they can stand on the floor. Additionally and in the near future, the patient will be continuously monitored with a load washer above the patient. The patient will be able to adjust the amount of load he/she wants to place onto their legs and on the floor by adjusting the controls from $0 \%$ to $100 \%$. A nurse can take over controlling the HLPR Chair from behind with a dual control set to assist and/or guide the patient through rehabilitation if necessary. [Bostelman, 2006]

\section{SUMMARY AND CONCLUSIONS}

This survey provides a review of example off-the-shelf lift and wheelchair products on the market today. Also, it provides a review of example research in these areas including advancements in intelligent wheelchair designs and capabilities. We have focused on lift devices, wheelchairs, the combination of wheelchairs and lift devices, rehabilitation and standards. What appear to be lacking, as determined by our research for this survey, are the following points regarding wheelchair dependents:

- There is limited or no research and development in the area of advanced patient lift devices, beyond standers, that are combined with wheelchairs to allow lift above approximately $5.5 \mathrm{~cm}$ (14 in) to reach upper shelves and even upper floors,

- There is limited or no research and development in the area of patient lift devices combined with wheelchairs to place patients on toilets, chairs, or beds or in bathtubs,

- There has been and is research and development ongoing in the area of advanced intelligent wheelchairs but, has yet to reach the commercial market due to: cost, lacking robust capabilities, little or no clinical trials, or other issues,

- There is advanced research ongoing to study navigation with 2 dimensional sensors such as LADAR (laser detection and ranging) but, minimal efforts toward 3D environment sensors use on wheelchairs beyond cameras is being studied,

- There are only limited wheelchair exercise equipment devices available and non are attached to the wheelchair to provide partial weight bearing by patients toward leg rehabilitation,

- There are limited patient lift devices on the market and none have intelligent capability to navigate in medical facilities on their own to "come when called" as needed by limited nursing staffs,

- There are no standards for intelligent wheelchairs and no standards that are guiding a concerted effort toward optimal intelligent wheelchair designs.

Bekey and Kumar presented the following information regarding assistive technology challenges at the National Science Foundation/National Aeronautics and Space Administration/ World Technology Evaluation Center Workshop [Bekey, 2005] in their presentation entitled, "Rehabilitation Robotics and Assistive Robots":

Challenges

Research

- Understanding human intention and adapting to it

- Control for human-in-the-loop navigation

Technology 
- Flexible input devices

- Inexpensive

- Why does a good wheelchair cost as much as a top-of-the-line BMW?

Challenges: Overarching

- No funding agency owns assistive technology

- U.S. Investments are not comparable to Europe, Japan

- Economics (insurance/health care)

\section{RECOMMENDATIONS}

Based on the above points, NIST recommends that the following research and development begin:

- Develop higher and more capable lift wheelchairs allowing WCD's to reach above currently achievable heights to be more independent at home and in the work place

- Develop wheelchairs that can place WCD's on toilets, chairs, beds and in bathtubs,

- Develop robust and affordable intelligent wheelchairs that are championed by medical funding agencies toward market potential,

- Develop advanced wheelchairs that use 3D environment sensors for robust world sensing and modeling to plan appropriate and safe robot paths,

- Develop advanced, partial weight bearing, rehabilitation devices that are integrated into wheelchairs for easy user accessibility and use by the wheelchair dependent,

- Develop patient lift devices that assist nursing staff through advanced mobility and lift capabilities to act a nursing assistant when there is limited nursing staff,

- Develop standards for intelligent wheelchairs that drive robust designs for the optimal mobility device for WCD’s.

Also, the following points should be addressed regarding funding for this R\&D:

- Develop US Government programs to provide standards, performance measurements and advanced technology development needed to reduce the healthcare assistive device costs to the public,

- Provide comparable investment in this area of technology to European investments. 


\section{REFERENCES}

[AAT, 2005] AAT Alber Antriebstechnik GmbH website: http://www.aat-online.de/de_home/

[Altimate Medical, 2005] Altimate Medical website: http://www.easystand.com

[Argyros, 2002] Antonis Argyros, Pantelis Georgiandis, Panos Trahanias, Dimitris Tsakiris, Semi-autonomous Navigation of a Robotic Wheelchair, Journal of Intelligent and Robotic Systems 34: 315-329, Kluwer Academic Publishers. Printed in the Netherlands.

[Arjo- Resident Gallery, 2005] website: http://www.arjo.com/int/Page.asp?PageNumber=2

[Arjo- Bari-Med, 2005] website: http://www.arjo.com/int/Page.asp?PageNumber=283

[Bellis, 2005] Bellis, Mary, "Wheelchair History,"

http://inventors.about.com/library/inventors/blwheelchair.htm

[Blevins, ????] Healthcare Statistics: Blevins Medical, Inc., http://www.patientlift.net/282164.html.

[Bostelman, 2006-1] R., Russo, P., Albus, J., Hong., T., Madhavan, R., “Applications of a 3D Camera Towards Healthcare Mobility Aids,” IEEE International Conference on Networking, Sensing and Control, Ft. Lauderdale, FL, April.

[Bostelman - 2006-2] R., Albus, J., "ROBOCHAIR - A Service Robot for the Healthcare Industry,” 3rd Int. Workshop on Advances in Service Robotics (ASER), Vienna, Austria, 6-7 July 2006. (submitted)

[Bostelman, 2004] R., Albus, J., “Lifter Design,” NIST unpublished design report, August 6.

[CALL Centre] Communication Aids for Language and Learning Centre, Scotland, http://CALLCentre.education.ed.ac.uk/Smart_WheelCh/What_is_it_SWA/what_is_it_swa.html\# collision

[ChicagoPT, 2005] http://www.chicagopt.com/

[Cooper, 2004] R., Simpson, R., LoPresti, E., Guo, S., Ding, D., (Principal Investigators), NIH Small Business Innovative Research Project, http://www.herlpitt.org/research.htm, 10/20029/2004

[Cooper, 2005] R., email to Roger Bostelman re. Intelligent Wheelchair Standards, July.

[Cooper, ????] R., Boninger, M., Algood, D., Cooper, R., "Impact of Pushrim Activated Power Assist Wheelchairs on Mobility Among People with Tetraplegia,” http://www.herlpitt.org/research.htm, University of Pittsburgh. 
[Cooper, 2004 -2] R., Rentschler, A., Vitek, M., Ammer, W., "Rehabilitation Engineering Research Center on Wheeled Mobility,” DOE/NIDRR Funded Project, 7/1999-6/2004.

[Duke University, 2004], Lisi, P., Steinberg, A., “The Lifting Assist for a Powered Wheelchair," Pratt School of Engineering, Duke University, http://www.resna.org/ProfResources/Publications/Proceedings/2004/Papers/StudentDesign/SM/L iftAssist.php

[HERL] HERL Laboratories, University of Pittsburg

[Hobson, 1999] D., "Development and Application of Wheelchair Standards: Everything most people want to know in 30 minutes or less!” presentation, RERC on Wheeled Mobility, Department of Rehabilitation Science and Technology, University of Pittsburgh

[KATO, 2005] H., Haak, R., “New Bipedal Robot,” A-Tip News article, http://www.uitec.ac.jp/rehb/welcome.html, May 17.

[Kim] C., Kim, S., and Kim, B., "RTAI Based Real-Time Control of Robotic Wheelchair," Guseong-dong, Yuseong-gu, Daejeon 305-701, Korea Department of Electrical Engineering \& Computer Science, Korea Advanced Institute of Science and Technology.

[Kuno, 2000] Y., Murashima, T., Shimada, N., Shirai, Y., "Intelligent Wheelchair Remotely Controlled by Interactive Gestures," International Conference on Pattern Recognition, vol. 04, no. 4, p. 4672.

[Marras, 1999], W., “Lifting Patients Poses High Risk for Back Injuries,” Ohio State University, http://researchnews.osu.edu/archive/resthome.htm.

[Miller,1995] D., Slack, M., “Tin Man I, Tin Man II,” Autonomous Robots \#2, pps. 77-88, KISS Institute, MITRE Corporation, Apple Computer, and Vector Mobility.

[Miller Engineering, 2001]

http://www.millerengineering.com/Example\%20Tasks/Res_Sum.htm, Ongoing Research Summary (Pediatric Sling Accident), July 12.

[NCMRR, 2003] “Innovations In Powered Mobility Devices,”: SBIR/STTR RFA: HD-03-023, National Institute of Child Health and Human Development (NICHD), http://www.nichd.nih.gov/, June 30.

[NIDRR/DOE, 2001] Bertocci, G., Fitzgerald, S., "Rehabilitation Engineering Research Center on Wheelchair Transportation Safety”, 11/1/01-10/31/06.

[OSHA, 2005] Henshaw, J., http://www.osha.gov/SLTC/nursinghome/solutions.html. 
[Patel, 2002] Patel, S., Jung, S-H., Ostrowski, J., Rao, R., Taylor, C., “Sensor based door navigation for a nonholonomic vehicle,” GRASP Laboratory, University of Pennsylvania, Proceedings of the 2002 IEEE International Conference on Robotics and Automation, Washington, DC, May.

[Palo Alto VA Health Care System] Jaffe, D., Presentation: “Assistive Technology for Persons with Disabilities,” Palo Alto VA Health Care System, Rehabilitation Research and Development Center, http://guide.stanford.edu.

[Pollack, 2004] M., “Intelligent Technology for Adaptive Aging” Presentation, AAAI-04 American Association for Artificial Intelligence Conference Keynote Address.

[Song, 1999] Song W.-K.; Lee H.; Bien Z.' KAIST - KARES: Intelligent wheelchair-mounted robotic arm system using vision and force sensor,” Robotics and Autonomous Systems, vol. 28, no. 1, pp. 83-94(12), 31, Publisher: Elsevier Science, July.

[Seiki, Fujitsu, 2005] Aisin Seiki Co., Ltd. and Fujitsu Limited, Intelligent Wheelchair- Tao Aicle, Expo 2005 in Aichi, Japan, March 2005.

[Stanford, 1987] Intex Industries - Alexis Wheelchair, http://guide.stanford.edu/Projects/CommlProd.html

[Stevens Publishing Corporation, 2006]

http://www.hhponline.com/Stevens/HHPPub.nsf/ProductView2?OpenForm\&category=Power+ Wheelchair

[Thomas, 2005] G., Tokyo Motor Show, March 2005 Issue.

[Toyota, 2005] Pod, Expo 2005 in Aichi, Japan, http://www.livingroom.org.au/robotics/archives/robotic_pods_take_on_car_design/, March 2005.

[U.S. Bureau of Labor Statistics, 1994] from Blevins website: http://www.patientlift.net/282164.html.

[University of Austin, 1998] Vulcan Robotic Wheelchair, http://www.cs.utexas.edu/users/qr/robotics/wh-picts.html

[University of Delaware, 2005] Photographs provided by Sunil Agrawal, Professor of Computer Science.

[University of Essex, 2004], "Researchers to develop intelligent wheelchair," http://www.innovations-report.com/html/reports/information_technology/report-29430.html and http://cswww.essex.ac.uk/staff/hhu/HCR-Group.html, May, 21. 
[University of Pennsylvania, 1994] Krovi, V., Wellman, P., Kumar, V., Harwin, W., "Design of a Walking Wheelchair for the Motor Disabled," Proceedings of the 4th International Conference on Rehabilitation Robotics, Wilmington, DE.

[van der Woude , 1999] "Biomedical Aspects of Manual Wheelchair Propulsion: The State of the Art II,” Volume 5, Assistive Technology Research Series,Edited by: L.H.V. van der Woude , M.T.E. Hopman and C.H. van Kemenade, 1999, 392 pp., hardcover.

[Wasatch Digital iQ, 2003] InTouch Health's Remote Presence Robot Used by Healthcare Experts, http://www.wasatchdigitaliq.com/parser.php?nav=article\&article_id=43, SANTA BARBARA, Calif. \& SALT LAKE CITY--(BUSINESS WIRE)--June 16.

[Wheelchair Standards Directory]

http://www.wheelchairnet.org/WCN_ProdServ/prodserv.html\#anchor9955825

[Whirlwind Wheelchair International, 2004] http://www.whirlwindwheelchair.org/

[Yanco 1] H., Hazel, A., Peacock, A., Smith, S. and Wintermute, H. "Initial Report on Wheelesley: A Robotic Wheelchair System,” Department of Computer Science, Wellesley College.

[Yanco 2] H., Gips, J., "Driver Performance Using Single Switch Scanning with a Powered Wheelchair: Robotic Assisted Control Versus Traditional Control,” Artificial Intelligence Laboratory Computer Science Department, Massachusetts Institute of Technology Boston College.

\section{Links}

The following are related department sites within the University of Pittsburgh Rehabilitation Science and Technology Department of the School of Health and Rehabilitation Sciences:

- Center for Assistive Technology: http://www.cat.pitt.edu/

- National Institute on Disability and Rehabilitation Research (NIDRR) Rehabilitation Engineering Research Center on Wheeled Mobility: http://www.rercwm.pitt.edu/

- $\quad$ RERC on Wheelchair Transportation Safety: http://www.rercwts.pitt.edu

- Pitt/VA Human Engineering Research Laboratories : http://www.herlpitt.org

- WheelchairNet: http://www.wheelchairnet.org

- Wheelchair Standards: http://www.wheelchairstandards.pitt.edu 


\section{APPENDICES}

\section{Appendix 1 - Lift Device Research Summary}

This excerpt was copied directly from [Miller Engineering, 2001] and provides a good sample of the safety organizations, statistics, other references and manufacturers of patient lift devices. It is provided here for convenience to the reader.

\section{Ongoing Research Summary (Pediatric Sling Accident)}

July 12, 2001

The following sources have been consulted and contacted for information related to the subject case:

\section{$\underline{\text { Standards/Regulations }}$}

Safe Medical Devices Act of 1990, October 5, 1990: An amendment to the Federal Food, Drug, and Cosmetic Act "to make improvements in the regulation of medical devices." Its purpose is "to improve the Medical Device Amendments of 1976, the basic law under which the Secretary of the Department of Health and Human Services...regulates the safety and effectiveness of medical devices.” (74.V.A.1.e)

Food and Drug Administration Modernization Act of 1997, Title II-Improving Regulation of Devices, January 7, 1997: This amendment to the Federal Food, Drug, and Cosmetic Act pertains to regulation of medical devices. Different sections of the Act discuss investigational device exceptions, special review for certain devices, expanding humanitarian use of devices, device standards, premarket notification, device tracking, and postmarket surveillance. (74.V.A.1.d)

Assistive Technology Act of 1998, Public Law 105-394, Nov. 13, 1998: Regulates the design of and access to assistive technology devices for the disabled. Assistive technology devices are "devices that facilitate activities of daily living, that significantly benefit individuals with disabilities of all ages.” (Sec. 2(4)). Lifts and slings would fall into this category. Proposes the use of "universal design principles" that could expand the use of assistive technology devices "by individuals with disabilities of all ages" (Sec. 2(10)). This act repeatedly stresses the right to assistive technology devices by disabled persons “of all ages.” Sec. 214(b), “Assistive Technology for Children and Older Individuals," allows states to provide financial assistance "for projects designed to increase the availability of assistive technology for populations of children and older individuals.” (74.V.A.1.c)

Code of Federal Regulations Title 21 - Food and Drugs, Part 801 - Labeling, April 1, 1999 Edition: Details label requirements for medical devices. (74.III.D.2) 
Code of Federal Regulations Title 21 -Food and Drugs, Part 803 - Medical Device Reporting, April 1, 1999 Edition: Details medical device reporting. "Under this part, medical device user facilities and manufacturers must report deaths and serious injuries to which a device has or may have caused or contributed.” (74.V.A.1.f)

Code of Federal Regulations, Title 21 - Food and Drugs, Part 880 - General Hospital and Personal Use Devices, April 1, 1999 Edition: Includes the identification and classification of a non-AC-powered patient lift (880.5510) (74.III.D.1)

Code of Federal Regulations, Title 21 - Food and Drugs, Part 860-Medical Device Classification Procedures, April 1, 1999 Edition: Defines the three classes of medical devices. Class I (which includes lifts with slings) means the class of devices that are subject to only general controls. Class I devices are not life-supporting or life-sustaining or for a use which is of substantial importance in preventing impairment of human health, and which do not present a potential unreasonable risk of illness or injury. Section 860.7 discusses the determination of safety and effectiveness of devices. (74.V.A.1.a)

Code of Federal Regulations, Title 21 - Food and Drugs, Part 812-Investigational Device Exemptions, April 1, 1999 Edition: Provides procedures for the conduct of clinical investigations of devices. (74.V.A.1.b)

\section{$\underline{\text { Statistics }}$}

Lift Injuries 1991 to Present, Consumer Product Safety Commission, National Injury Information Clearinghouse, report dated June 1, 2001: According to CPSC accident investigations, reported incidents and/or death certificates, along with data from the National Electronic Injury Surveillance System (NEISS), 116 people were injured and 11 were killed from 1991 to the present in incidents involving lifts. (74.V.B.3.c)

University of Michigan, "Anthropometry of Infants, Children and Youths to Age 18 for Product Safety Design, " U.S. Department of Commerce, National Technical Information Service, May 1977, p. 428-435: Provides seated center of gravity statistics for both males and females in different age categories beginning with age two and ending with age nineteen. The mean seated center of gravity for a seven-year-old (the age of the child in this case) is $20.4 \mathrm{~cm}$. The mean seated center of gravity for a nineteen-year-old is 25.1 cm. (74.V.B.3.a)

U.S. Food \& Drug Administration, Center for Devices and Radiological Health, MAUDE Database: Provides injury/adverse event statistics for medical devices. Included here are injuries due to the use of an incorrect sling in a lift device. In the database section covering 1996-present, more than 500 records pertain to slings. Six of the records document injuries due to use of an incorrect sling or sling size. In the section covering 1992-1996, 135 sling incidents are recorded. Four incidents involve the use of an incorrect sling or sling size. All ten incidents occurred in a hospital or other health care facility. Seven of the incidents involved a lift/sling device manufactured by Arjo. The 
three other devices involved were manufactured by Invacare, Guardian and Hoyer. (74.V.B.3.b)

\section{$\underline{\text { Online Articles/Resources }}$}

Duncan, Janell Mayo, “Legal and Policy Issues Relating to the Use of Medical Devices in the Home, and the Home as a Health Care Setting," HCT Workshop Discussion Paper: Discusses major legal and policy issues relating to the home as a health care setting, including 1) Federal regulation of medical devices used in the home; 2) Federal reimbursement policies for the provision of home care and telemedicine services; 3) tort liability concerns; and 4) access to home care and telemedicine services. (74.V.b.6.d)

Agency for Healthcare Research and Quality, "Medical Errors: The Scope of the Problem," Publication No. AHRQ 00-P037, Agency for Healthcare Research and Quality: Discusses where errors occur, noting "care delivered in the home" in addition to traditional health care settings. Looks at the costs and seriousness of the problem. Lists the types of errors, including "misinterpretation of other medical orders." (74.V.B.6.e)

Schuch, Jonathan Z. and Betty H. Breeden, “Managing the Legal Liabilities and Risks Inherent in the Delivery of Assistive and Rehabilitation Technology Services and Devices," RESNA Proceedings, 1996: Describes "strategies for effectively managing the legal liabilities and risks associated with the delivery of assistive and rehabilitation technology (AT/RT) services and devices." The article states: "Because services and products are delivered to consumers, assistive technology practitioners and rehabilitation technology suppliers assume significant legal responsibility for their actions, to the extent that the consumer has a legally enforceable right to AT/RT services that meet or exceed professional standards and to AT/RT products that will not cause injury under normal use” (p. 1). Discusses professional liability and products liability. Notes that "a seller or supplier is subject to liability for negligence relating to his or her function in the distribution of a product” (p. 2). Presents eleven strategies to effectively managing liability. (74.V.B.6.b)

“Improving Patient Care by Reporting Problems with Medical Devices," A MedWatch Continuing Education Article, Dept. of Health \& Human Services, September 1997: Defines the term "medical device," discusses the premarket review and postmarket surveillance of devices, explains how to identify and avoid problems with medical devices, and describes the process of reporting device-related problems. Notes that healthcare professionals need to "understand how a device should be used, and for which patients it is probably not safe” (p. 3). (74.V.B.6.c)

U.S. Food and Drug Administration, Requirements of Laws and Regulations Enforced by the Food and Drug Adminstration, "VI. Medical Devices,": Summarizes regulations put forth in the Federal Food, Drug and Cosmetic Act and its amendments pertaining to medical devices. Discusses classification of devices, premarket notification for new devices, requirements for all devices, adulteration, misbranding, device registration and 
listing, good manufacturing practice regulations, banned devices, investigational devices, humanitarian devices, custom devices, and imported and exported devices. (74.V.B.6.a)

Rehabilitation Engineering Research Center on Technology Transfer, Center for Assistive Technology web site: Includes links to related sites, mission statement, strategic goals and contact information. (74.V.B.6.q)

\section{$\underline{\text { Journal Articles }}$}

Dobbs, Jean, “Transfer Devices and Lifts,” New Mobility, Vol. 6(20), March-April 1995, p. 55-56: Discusses transfer devices, lifts and slings. Notes that "Improvements in sling design in the last several years have reduced pressure sore problems associated with poor fit” (p. 56). (74.V.B.4.a)

Scalise, Mardi, “Lift Your Spirits,” New Mobility, Vol. 7(30), March 1996, p. 72: Provides information about specific brands of lifts, lift devices, and transfer devices, including the price. (74.V.B.4.b)

“Special Feature: Lifting \& Handling Equipment,” Disability View Magazine, May-June '99, $M$ \& S Publicity Ltd., c1999: Discusses how to find out what you need, hiring a home care company, bathing, slings, equipment providers, and choosing equipment. Notes that "Choosing the correct equipment, of course, is vitally important." Under the "Slings" section, it states, "Hoists are often only as good as the slings used with them; the choice of sling is one of the most important factors affecting the use of the hoist and the comfort of the person being lifted” (p. 3). (74.V.B.4.d)

Moody, Janis et al., “A Study of Nurses’ Attitudes Towards Mechanical Aids [Lifting and Handling]," Nursing Standard, Vol. 11(4), October 16, 1996, p. 37-42: Researchers interviewed one hundred and eighty-five nurses from a selection of specialties about their attitudes toward mechanical aids for lifting. Common problems identified were lack of proper training in mechanical aid use, mechanical aids and slings being unavailable or inaccessible, arbitrary use of mechanical aids, and spatial constraints to mechanical aid use. The article mentions a 1993 study by McGuire and Dewar in which "56 percent of nursing personnel felt that the amount and type of mechanical aids available did not match their clients' needs." Regarding slings, the article states: "The provision of an adequate supply of slings in a range of sizes is essential for the correct use of mechanical aids” (p. 7). Among the conclusions the authors reach are compatibility with other equipment should be taken into account when purchasing mechanical aids; training in, and adherence to, ergonomic principles in moving and handling is advocated in addition to the use of risk assessment and care plans; training programs should be specifically tailored to mechanical aid use, and "It is particularly important to train nurses to fit slings correctly.” (74.V.B.4.e)

Deane, Robin, “Caring for Kids,” Home Health Care Dealer Provider, May 2000:

Discusses pediatric home care. States that "Although geriatric and pediatric patients may have the same condition...equipment needs will likely not be the same because of size 
and personality development issues." Also notes that "Many home health care organizations are experts at providing care for the elderly but are not as equipped or knowledgeable to provide specialized care for pediatrics." Includes discussion of PedsLink, a company that offers home care providers the information and tools needed to start or improve a high-quality pediatric program. (74.V.B.4.c)

Wahl, Margaret, "Save Your Back! A Guide to Lifting and Transferring at Home," Quest, Vol. 6(1), February, 1999: Utilizes text and photos in explaining how to use a sling. Discusses floor and ceiling model lifts. (74.V.B.4.g)

\section{Book Excerpts}

Healthy People 2010: Objectives for Improving Health, “Chapter 17: Medical Product Safety, U.S. Food \& Drug Administration: This chapter's goal is to "ensure the safe and effective use of medical products.” It mentions MedWatch, the FDA's medical products reporting program. It describes the four categories of medical product risks: product defects, known side effects, medication or device errors, and remaining uncertainties. On device errors, the chapter notes that "Errors also involve unintended substitution of the wrong product for the prescribed product...Substantial numbers of injuries and deaths occur annually because of medication or device errors” (p. 17-6). The chapter also discusses risk communication and confrontation, and lists objectives for safer health care. (74.V.B.5.a)

Scherer, Marcia J., Living in the State of Stuck: How Technologies Affect the Lives of People with Disabilities, "Chapter Seven: Dilemmas, Challenges and Opportunities," Brookline Books, c1993: This chapter discusses the need for a model when matching a person and a technology (MPT), characteristics of the milieu, milieu factors influencing device use, characteristics of the person, and characteristics of the assistive technology. It is noted that by consulting a model such as the MPT model, one can help provide the rationale for funding a device or training for that device, demonstrate an individual's improvement in functioning over time, and help organize information typifying the needs of clientele. (74.V.B.5.b)

\section{Manufacturers/Dealers of Slings/Lifts}

There are many web sites for manufacturers of slings/lifts in general. Listed below are the major ones. Fewer companies deal in pediatric slings/lifts. The following are the pediatric dealers:

Barrier Free Lifts Inc.: They manufacture a variety of slings and lifts for both adults and children. The Tonya Lift for pediatric use is a smaller version of their Motorized Patient Positioner, in which the smaller size "enables the sling to better conform to children, ensuring safety and comfort.” Mobility Concepts Inc. is a distributor of Barrier Free Lifts products. Midwest Barrier Free Lifts is another distributor. (74.III.F.1.a\&b, u) 
Vancare Inc.: Their VANDER-LIFT 450 has a "heavy-duty frame with expandable base [that] offers tip-resistant support for residents/patients from pediatric to 450 lbs." Their "variety of durable slings meet size requirements to 450 lbs. and are comfortable for all residents/patients regardless of most range-ofmotion limitations.” (74.III.F.1.f)

Convaid Inc.: Listed in the Rehabmall.com web site, Convaid is a manufacturer of mobile positioning systems for children and young adults. (74.III.F.1.e)

Innovative Products Inc.: Also listed at Rehabmall.com, they claim, “Our equipment gives children the opportunity to experience freedom and mobility for the first time." They build to specification, depending on individual needs. (74.III.F.1.e)

Abilitations: Listed at Rehabmall.com, they publish a 192-page catalog of products with a focus on children's rehabilitation and therapy. "Products are available for sensory integration, movement, positioning, exercise and play." (74.III.F.1.e)

Guldmann: Their "Lifting Accessories” brochure includes guidelines on choosing the size of a sling. The sizing chart includes specifications for children. (74.III.F.1.r\&s, y)

The following are some major sling manufacturers: (74.III.F.1.g)

Ted Hoyer and Company (A Division of Sunrise Medical): They manufacture a transfer lift sling, one-piece sling with headrest, one-piece split sling, and a twopiece sling.

Invacare Corporation: Manufacturer of the subject sling. They manufacture a two-piece sling, one-piece contour sling, and a headrest sling. (also 74.III.F.1.t, x)

Barrier Free Lifts Inc.: They manufacture a universal sling, universal head support sling, two-piece quick sling, three-piece quick sling, Lexa sling, closed seat sling, full stretcher and partial stretcher sling, Patron Tubular sling, and the Dolphin Patient Positioner.

Waverlyglen Systems Ltd.: They manufacture a hammock sling, universal sling, positioning sling, hygiene sling, stretcher sling, and a walking sling.

The following are lift system manufacturers: Apex Dynamics Healthcare Products, Arjo Inc., Platinum Healthcare Equipment, Portable Entry Systems, Rand-Scot Inc., V. Guldmann A/S, Wy'East Medical Corporation, Columbus McKinnon Corporation, DayLight Technologies Inc., Medi-Man Rehabilitation Products, Mobility Transfer Systems, Patient Handling Technologies, Inter Care Scandinavia A/S, Ginco Medical Equipment, SureHands International, and BHM Medical (74.III.F.1.a-q, v, w) 


\section{Supporting Organizations}

This project was supported in part by:

* $\quad$ The Paralyzed Veterans of America (PVA)

* The National Institute on Disability \& Rehabilitation Research (NIDRR)

* The Rehabilitation Engineering Research Center (RERC)

* Human Engineering Research Laboratory http://www.herlpitt.org/ 


\section{Appendix 2 - Patent Search - "Patient Lift” AND "Wheelchair"}

A patent search was completed on October 20, 2005 through the on-line US Patent and Trademark Office website: www.uspto.gov. Keywords "patient lift" AND "wheelchair" returned 59 patents with the following as a representative sample:

United States Patent $\quad 6,715,167$

Wake April 6, 2004

Patient lift and transfer apparatus

Abstract

A two piece incorporated device designed to assist in the lifting and transfer of such person who is partially incapacitated by infirmity or disability from one sitting position in one location to another sitting position in another location. (ex: wheelchair to toilet, bed to chair, etc.) The device includes a torso belt with hold and support handles on back, secured by sewn on hook and loop style fastener; and a thigh support segment with handles sewn into either end, which supports the posterior and weight of said patient. The connection of these two pieces with quickdisconnect buckles provides a greater sense of balance for patient and attendant(s) and helps eliminate weight stress on the patient by virtue of proper weight distribution. The thigh support segment is also equipped with forward positioning straps (at either end), which facilitate a rocking forward by the attendant(s), bringing patient closer prior to lifting.

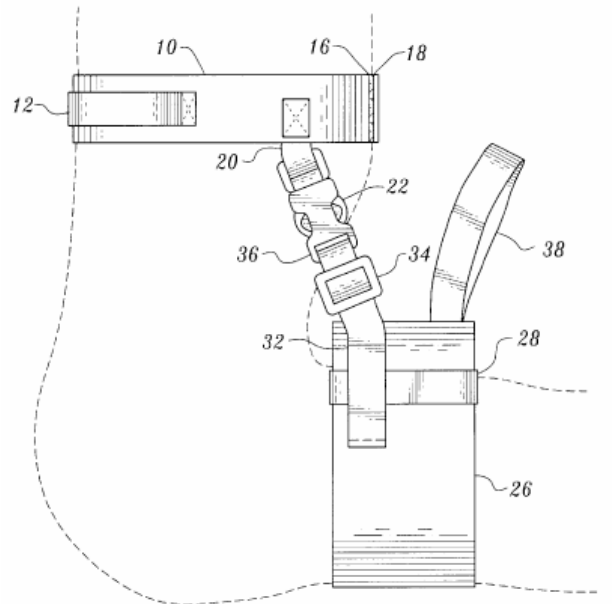

Fig. 1

United States Patent $6,637,610$

Cheeseboro October 28, 2003

Personal transporter

Abstract

Structure and procedures for lifting, transferring and moving a person, such as a bed ridden person or one requiring the use of a wheelchair, involve a frame (393) supported on wheels (394, 395), a horizontal load supporting beam (392) in an upper part of the frame, and a motor- 
powered winch (391) supported by the frame (393) for movement along the beam. Med winch (391) can be arranged to synchronously raise and lower a pair of horizontally spaced couplings by which a carrier (480) for a person can be connected to the winch (391). The beam (392) can be of variable length. The frame (393) can include a pair of beam-supporting legs (406, 407), one of which can be movable along and removable from the beam as extended from a minimum length state in which the frame (393) can be moved through a doorway. The frame (393) can be of fixed width, with the beam extendible from a side of the frame in conjunction with groundengaging stabilizers $(446,446$ ') which are extendible from the frame to support the frame from overturning when a person is supported outside the frame from the extended beam (413). A carrier (480) for a person can be a fabric construction which has stiffened back (483) and seat portions, and a lift point at each side of the carrier (480). Another form of carrier can also serve as a portion of a bed.

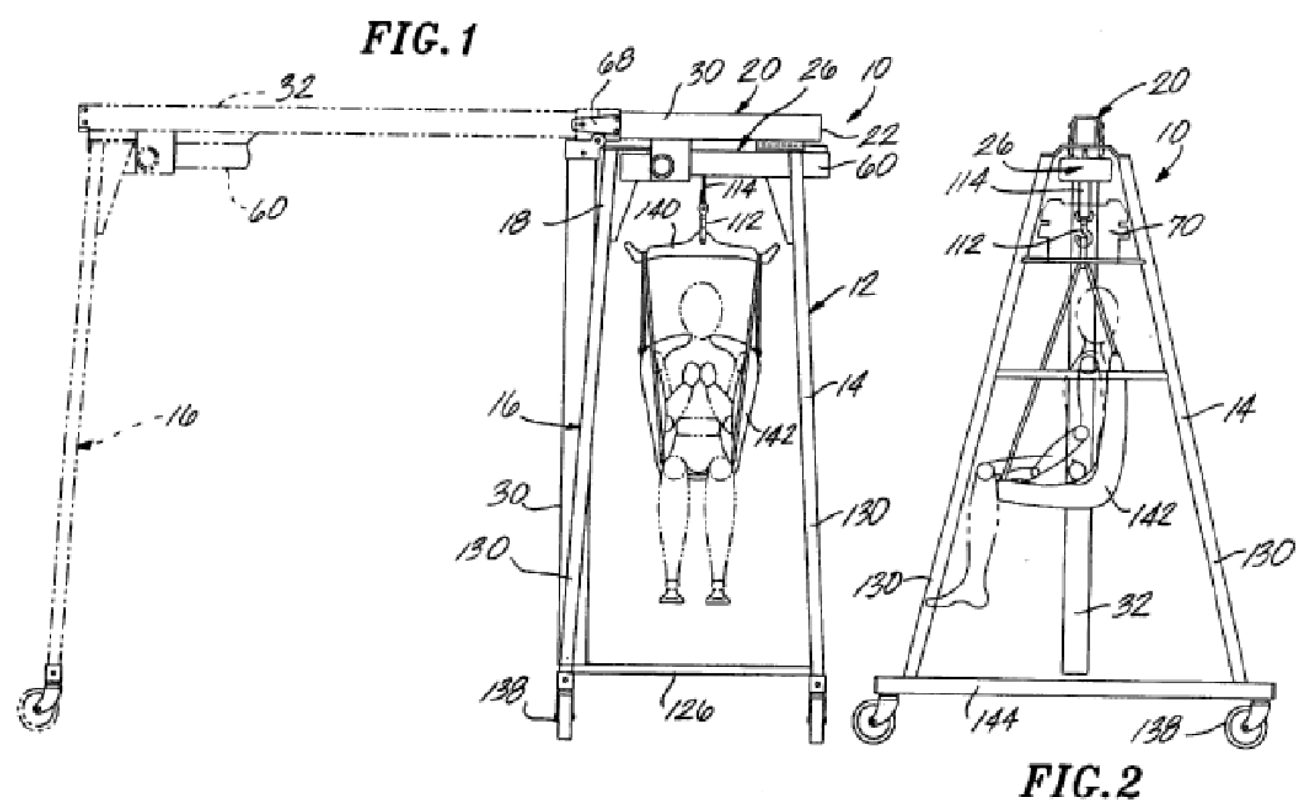

United States Patent $6,454,285$

Koenig September 24, 2002

Ergonomic wheelchair with patient lifting mechanism

\begin{abstract}
A wheelchair for use in care facilities includes a seat lift mechanism and a chair tilt mechanism both of which are actuated by a single lever. Operation of the lever elevates the rear of the seat and raises the rear of the wheelchair thereby moving the wheelchair occupant toward a standing position and reduces the lifting effort of the attendant in assisting the debilitated patient to move out of the wheelchair.
\end{abstract}


Draft: 9/15/06

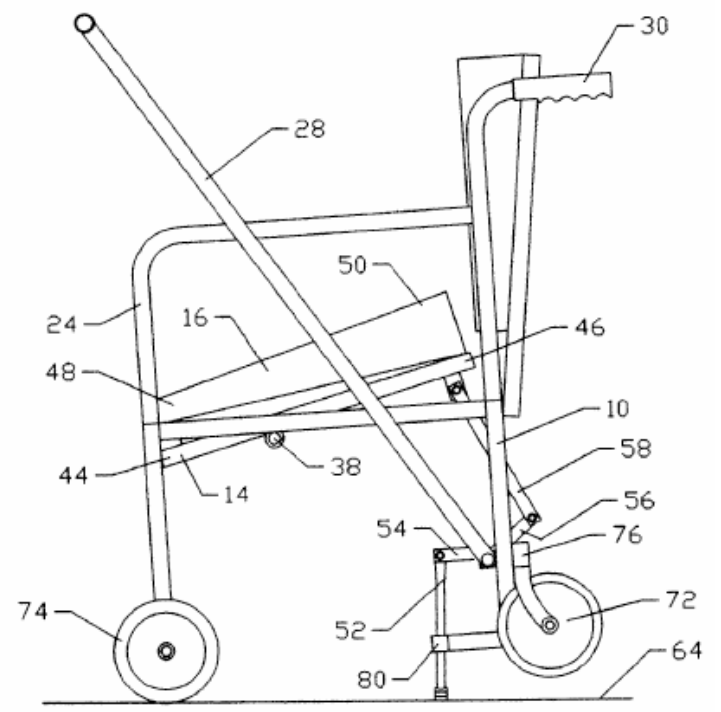

United States Patent $6,430,761$

Brandorff, et al. August 13, 2002

\section{Compact portable patient lift}

Abstract

An improved portable patient transfer device comprising a small wheeled base having a vertically disposed telescoping lifting column extending upwardly therefrom. A patient support means extends from the column to support the patient. A crank, accessible to both patient and attendant, is used to raise and lower the lifting column for transfer between surfaces with different heights, such as beds, wheelchairs, and car seats. The lift my be closed and temporarily attached to a wheelchair for convenient transport with a patient. The lift is small and lightweight and may be easily transported and stored. The lift is further economical to manufacture.

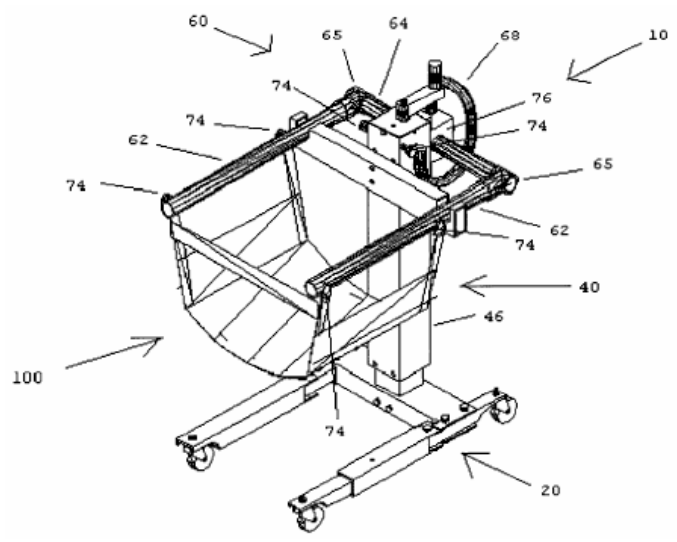

United States Patent 5,809,591

Capaldi , et al. September 22, 1998

Patient lift mechanism 


\section{Abstract}

A patient lift system incorporates a transverse bar which carries a patient lift system for movement between two laterally extending bars. The transverse bar is mounted at the same vertical height as the laterally extending bars and carries a motor for lifting and lowering the patient. Since the transverse bar is at the same vertical height additional patient lift height is provided as compared to prior art systems. In a further feature of the present invention, the frame includes an improved corner bracket and also includes vertical adjustment for the legs to achieve leveling of the overall frame. In another feature of this invention, a patient lift bar includes four lift points, with two forward lift points spaced by a greater distance than the two rearward lift points. This provides greater support to the rear of the patient, while the additional distance in the front facilitates entry and removal of the patient lift system. In addition, an inventive sling provides a support for attachment to the lift bar which comfortably lifts the patient. In other aspects of this invention, a solid state motor control allows easy control of the speed of the motor for the patient lift system. In other features, the motor control incorporates a remote control that receives a low voltage and current such that the remote control is relatively safe to use. In a second embodiment sling, a head portion supports the patient's head, and is to the rear lift points described above. Other improvements are disclosed in the application.

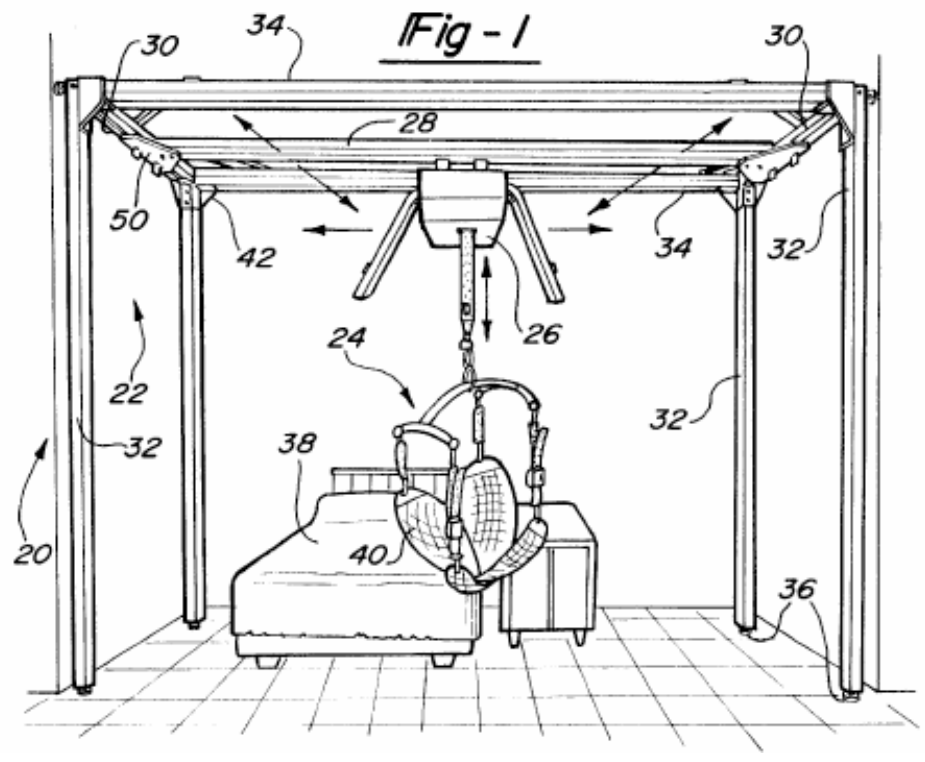

United States Patent 5,694,654

Roy December 9, 1997

Patient lifting and transfer system

Abstract

A lifting and transferring system for lifting a person and transferring the person from one location to another location comprises a lifting and transferring assembly and a transfer apparatus. The lifting and transferring assembly comprises a lifting arm assembly and a support, the lifting arm assembly providing a lifting arm rotatable about a vertical axis. A mounting rotatably mounts the lifting arm to the support so that the lifting arm cantilevers from the 
mounting. An extendable and retractable lifting cable depends from an outer end of the lifting arm. A first motor is provided for rotating the lifting arm. A second motor is provided for extending and retracting the lifting cable. A controller is provided to operate the first and second motor. The transfer apparatus is connectable to the lifting cable and arranged to support the person during operation of the lifting and transferring assembly.

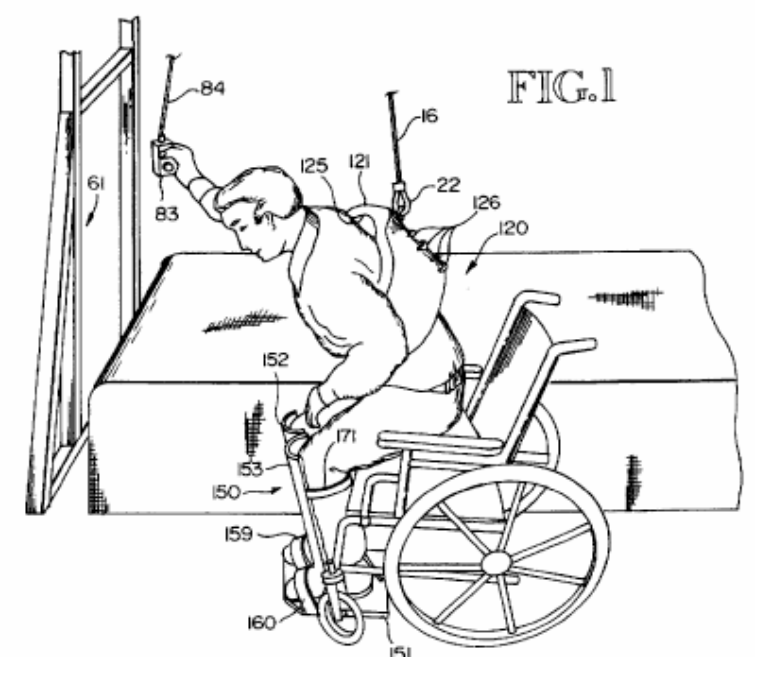

United States Patent 5,520,403

Bergstrom, et al. May 28, 1996

Wheelchair with translating seat and patient lift

Abstract

A wheelchair having a set of features which facilitate transfering a patient to an alternative surface and raising the chair to an alternative seating height. The first of these features enables each of the handwheels to be independently pivoted rearward by raising its related arm rest. The motion of the wheel is over centering and its rearward position is determined by a brake pad which engages its respective handwheel. The rearward position of the handwheel is such as to clear the rear of the seat. The forward position is determined with the arm rest in the normal down position and latched to the frame. The second feature is a lifting mechanism for raising the patient to the level of an alternative surface such as a bed. The third feature has the seat surface fabricated from a moveable belt which laterally transports the patient to the alternative surface. The wheelchair also has an embodiment which allows folding for easy transport. 
Draft: 9/15/06

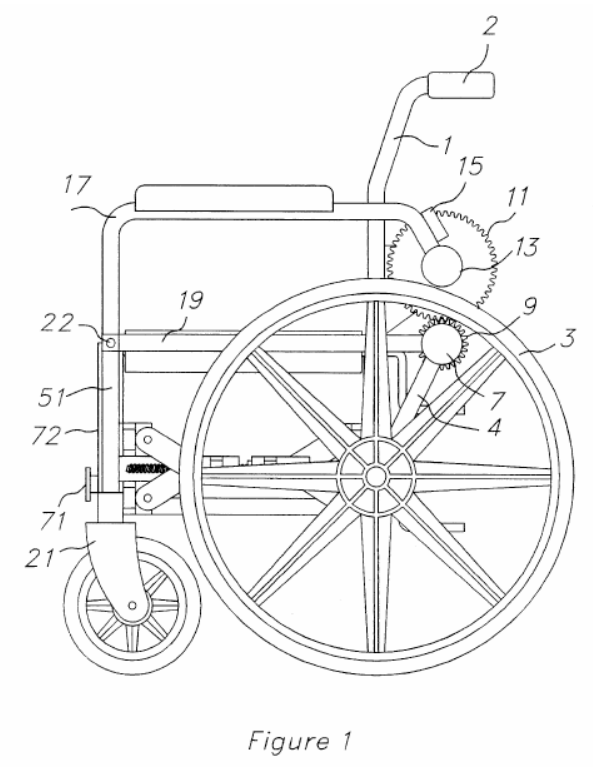

United States Patent 5,411,044

Andolfi May 2, 1995

Patient transfer walker

Abstract

A walker for a handicapped person has a pair of base rails juxtaposed, and a pair of front posts extend therefrom. Each front post is attached to a front of a respective rail. A first pair of rotation-changeable motors drives a pair of actuators, initially pointing upwardly. A first pair of gear reduction devices, which in combination with a pair of rear posts, can be rendered tiltable, has respective input shafts coupled to respective output shafts of the motors. The pair of tiltable rear posts are extendable or retractable by rotation-sense selection of the first pair of motors. A pair of crutch-supports are in contact with the rear posts, Which fit under the armpits of the handicapped person. A pair of levers which extend outwardly and downwardly from the pair of crutch supports, respectively, are bent rearwardly, and equipped with handles. A second pair of rotation-changeable motors are attached to the pair of front posts, respectively, and a second pair of length-adjustable gear-reduction mechanisms are coupled to the second pair of motors, and also to the pair of the rear posts, respectively, so as to be operative, upon length adjustment, between forward-and rearward-positions. A switch is connected to the first and second pairs of motors for separate selection of the height and inclination of the rear posts, respectively, by selective choice of the motor's sense-of-rotation and travel. 
Draft: 9/15/06

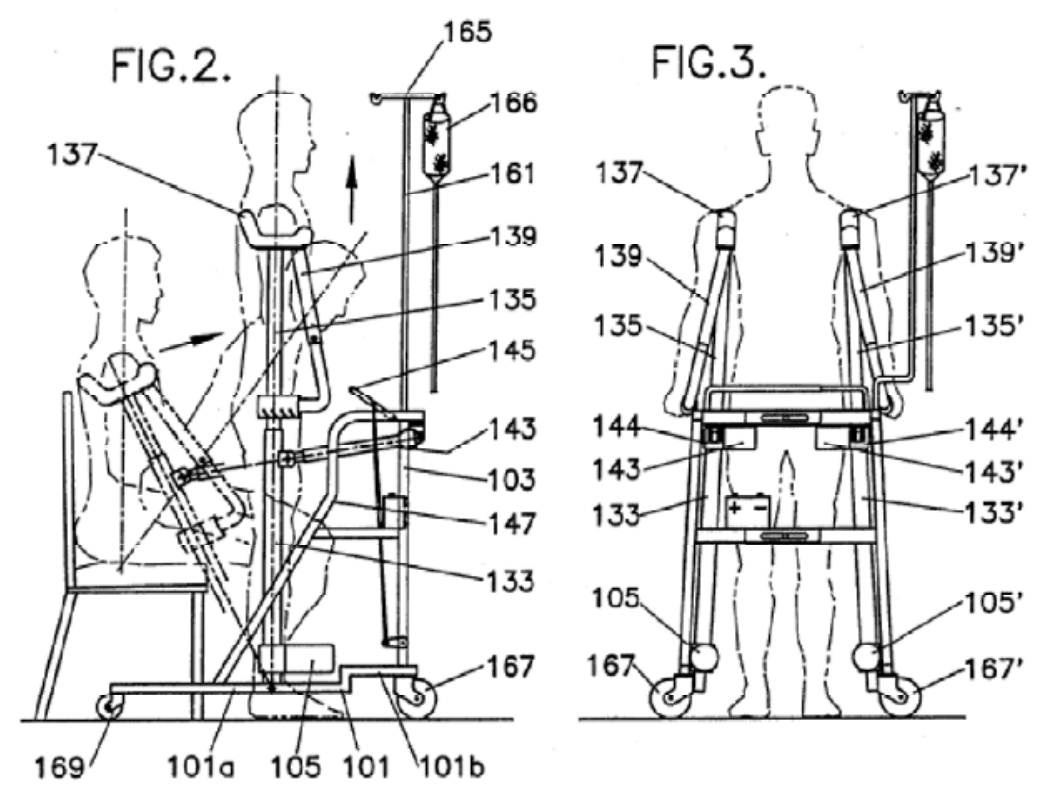

United States Patent $3,962,737$

James June 15, 1976

Lifting means

Abstract

An invalid hoist has a wheeled chassis with a rear mounting for an upstanding column and lifting arm. The chassis comprises a cross member with main load-carrying wheels adjacent the ends thereof and three fore-and-aft wheel-carrying arms projecting from the cross member. Two of the arms extend forwardly in spaced relation to leave an opening between them and the third arm extends rearwardly to support the column.
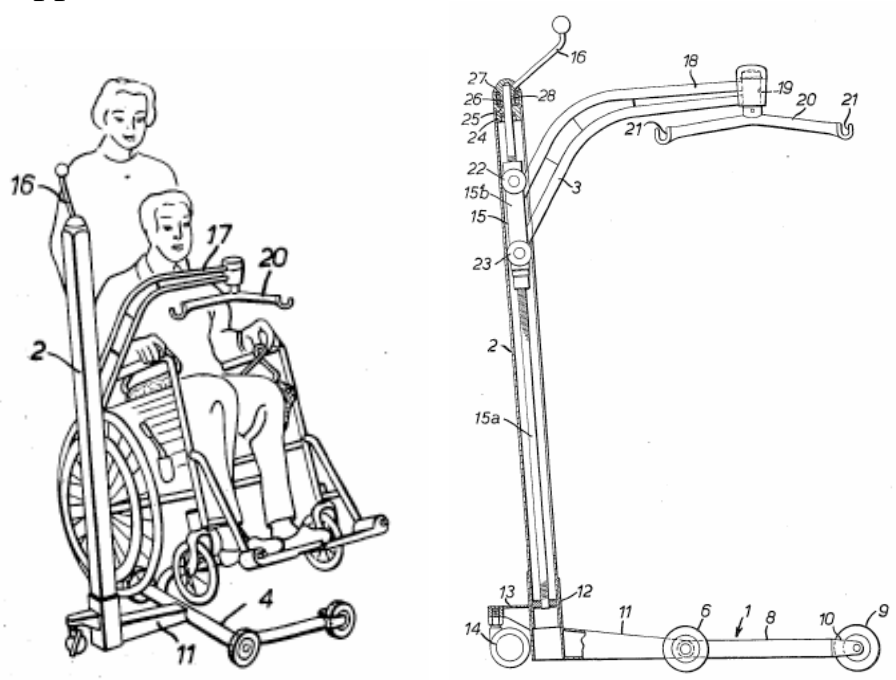


\section{Appendix 3 - Lift Devices List}

This patient lift devices list is from the United Spinal Association, Copyright 2004 and is shown here for the readers convenience. It can also be accessed on the world wide web at:

http://www.usatechguide.org/techguide.php?vmode=1\&catid=2 where a long list of categories can be searched regarding medical equipment.

Access Unlimited

The Mini Touch compact, steering wheel mounted control pad/steering knob. Transfer assist devices for getting in and out of vans, cars, and trucks.

(800) 849-2143

Accessible Environments

Bathroom safety equipment.

Apex Dynamics

Manufactures patient lifts, transfer products and shower chairs.

$\underline{\text { Arjo }}$

Systems for personal hygiene, lifting and transport, as well as therapy.

Arycare

The AryCare Home Unit will lift and transfer patients up to 500 lbs. The unit can remain in place over an average length bed. Only a standard wall outlet is necessary for power.

800-342-9018

Barrier Free Lifts

Floor, stretcher and ceiling mounted patient lifters.

800-582-8732

Bath Knight

Bath-Knight tub access device.

$\underline{\text { Bhm }}$

Patient lifts, track lifts, floor lifts, wall mounted lifts, bariatric lifts, slings and accessories.

Blevins Medical, Inc.

Offers the Versatran Lift. A platform based patient lifting and transferring system. Requires no slings and is multi-positional.

(540) 436-8395

\section{Borringia}

Chameleon portable shower commode chair. Dawn Care Hoists for tioleting. Transport chairs, lap trays, positioning devices, and Rise elevating \& tilting shower commode chair.

Can-dan Rehatec, Ltd 
Offers a bath support for children, adjustable changing tables, wheeled walking supports, and the Meyland Car-Lift transfer device.

905-648-7522

\section{$\underline{\text { Chiltern }}$}

Products - including Shower Trays and Enclosures, Trolleys and Stretchers, Easy Access Baths, Ceiling Mounted and Mobile Hoists.

Convaquip

Manufacturer and distributor of bariatric beds, bathroom safety equipment, walking aids, gurneys, lifts, mattresses, transport chairs and stretchers, and manual and powered wheelchairs. 800-637-8436

\section{Faaborg Rehab Technic}

Produces patient lifting systems. The lifter is produced in 15 different types to cover every need. Floor and ceiling track models.

$\underline{\text { Ferno }}$

Therapy pools, underwater treadmills, underwater exercise machine, lifts.

Gaper

Patient lifting devices. Portable ceiling track lift, permanent ceiling track lift, mobile floor lifts, motorized carriage, and sling systems.

(905) 820-0004

Guldmann

Patient lifters- ceiling track lifts, mobile floor lifts, beds, and vertical platform lifts.

Handicap Helpers Inc.

The Lizzie Lift is a patient lift and transport device. The device is mobile and can go from a sitting to a stretcher position.

(276) 565-1889

\section{Invacare}

Manufactures and distributes manual and powered wheelchairs and seating and positioning aids, pressure reducing surfaces, ambulatory aids, resporatory equipment, patient lifts, and homecare beds.

\section{Lift Aid Inc}

Gantry type patient lifter. Lift Aid 2000 is a lifting and transferring system.

(248) 348-1000

\section{Liftup}

The Liftup, an ADL-assist device that lifts a disabled person into position for transfers in bathroom, bedroom or other locations. 


\section{Liftvest Usa}

Liftvest, aid in wheelchair transfer and walking stability that increases patient security and comfort, while minimizing caregiver backstrain.

212-874-2499

$\underline{\text { Liko }}$

Develops and manufactures mobile and overhead patient lifts for an international market. 888-545-6671

Mangar International

A range of unobtrusive small portable lifts for home, in residential care or in hospital.

Maxhealth Corporation

OEM of walking aids, shower commode chairs, wheelchairs, patient lifters, and pressure reducing surfaces.

$\underline{\text { Med-fit }}$

Mobile patient lifts and transporters.

800.831.7665

Merivaara

The Merilift series includes ceiling hoists and movable hoists for use in nursing homes, hospitals and private residences.

$\underline{\text { Molift }}$

Manufacturer of patient lifters. The Molift Smart- a folding lifter. Quick Raiser- for transfers and standing. The Partner lift. Hi Trac- a ceiling mounted track lift. Quick Mover- lifts from underneath avoiding obstacles around the head. Also manufactures stretchers, scales, pool lift, trapeze and standing tables.

\section{Noram Solutions}

Offers recumbent and seated bathing systems, patient lifters, ceiling track lifts, and sit to stand lifts.

800-387-7103

Paramobility

Portable patient lifters for swimming pools and vehicles.

\section{Pro Med}

Designs and manufactures a range of patient lifters, slings, and frames for use in Hospital, Nursing Home, or private home care.

+61395320466

\section{$\underline{\text { Sizeright }}$}

SizeRIGHT.com offers heavy duty medical equipment and products for larger sized individuals in a facility or home care setting. 
888-829-1009

\section{Sunrise Medical}

Quickie brand wheelchairs, Jay Cushions, Smartseat seating systems, bathroom satety equipment, walking aids, patient lifts, pressure reducing surfaces, and DeVilbiss brand respiratory equipment.

(303) 218-4500

$\underline{\text { Surehands }}$

Range of Handi-Move products and lifts.

(800) 724-5305

\section{T. H. E. Medical}

Manufactures and distributes a line of bathing and handling devices. Includes recumbent bathing equipment, patient lifts and transporters, and slings.

800-565-7075

\section{Take Along Lifts}

Portable patient lifters.

877-667-6515

\section{Tr Equipment}

Bathing and shower equipment, patient lifters, gait training equipment, and walking aids. Shower gurneys and elevating baths.

\section{Tuffcare}

Product line includes manual wheelchairs, power wheelchair, freedom carts, patient care products and homecare beds, walking aids, and bathroom safety devices.

Waverlyglen Systems

Designs and manufactures ceiling lifts.

White Medical Services, Inc.

The Koziwrap Lift System is a lift system for the handicapped person and is used with a manual or hydraulic lift device.

(866) 944-8373

Whittiker Company

Lifts, elevators, platform, inclined, vertical. Stairway Lifts, Elevators, Bathtub Lifts, Inclined Platform Lifts, Vertical Platform Lifts, Ceiling Lifts and Wheelchairs.

800-445-4387

Wy East Medical

Offers patient lifts and transfer devices. 


\section{Appendix 4 - Patient Characteristics List}

Arjo, Inc. provides an excellent list of patient characteristics. They are listed below for the readers convenience and provide a way to categorize mobility, dependency on the caregiver, and what abilities need stimulation. Following this list is a similar list focused on Bariatric patients. Arjo provided drawings simulating each characteristic type to visually assist in matching a patient type to a particular characteristic. [Arjo, 2005] To make the list more associated to humans, the list is labeled A through E with each letter used to begin a name of a particular patient characteristic type.

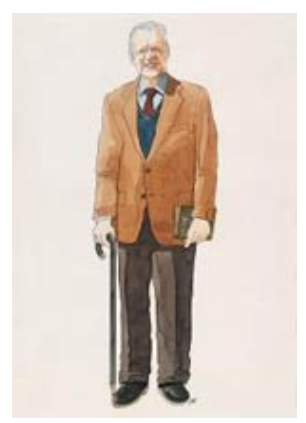

$\underline{\text { A (Albert) }}$

- Ambulatory, but may use a cane for support

- Independent, can clean and dress himself

- Can tire quickly

- Stimulation of abilities is very important

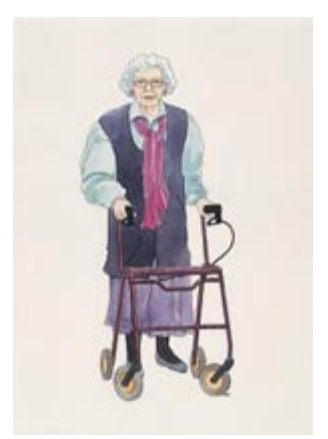

$\underline{B}$ (Barbara)

- Uses walking frame or similar

- Can support herself to some degree

- Dependent on carer who is present in demanding situations

- Not physically demanding for carer

- Stimulation of remaining abilities (e.g. ambulation) is very important 


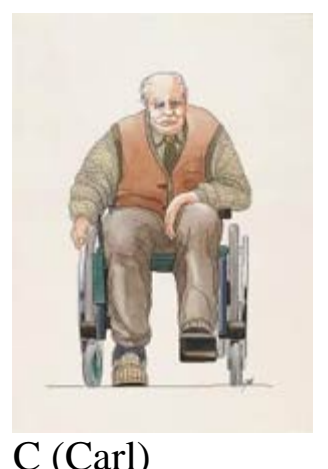

- Sits in wheelchair

- Is able to partially bear weight on at least one leg

- Has some trunk stability

- Dependent on carer in most situations

- Physically demanding for carer

- Stimulation of remaining abilities is very important

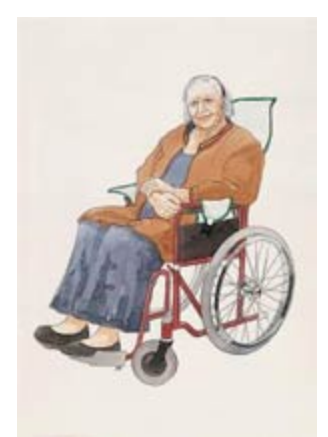

\section{$\underline{\mathrm{D} \text { (Doris) }}$}

- Sits in wheelchair

- No capacity to support herself at all

- Cannot stand unsupported and is not able to bear weight, not even partially

- Dependent on carer in most situations

- Physically demanding for carer

- Stimulation of remaining abilities is very important

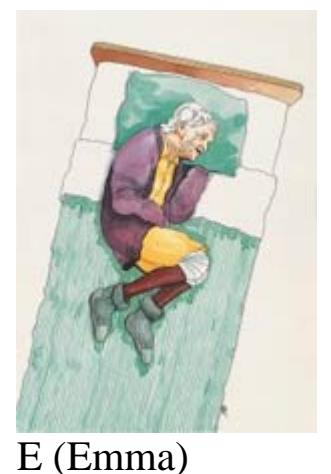

- Passive resident

- Might be almost completely bedridden

- Often stiff, contracted joints 
- Totally dependent

- Physically demanding for carer

- Stimulation and activation is not a primary goal

\section{$\underline{\text { Bariatric Patient Characteristics List }}$}

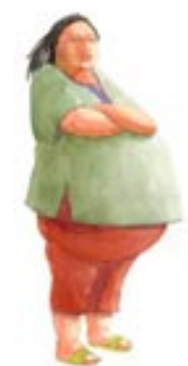

\section{A (Adisa)}

- Ambulatory, but may use a cane for support

- Independent, can clean and dress herself

- Can tire quickly

- Stimulation of abilities is very important

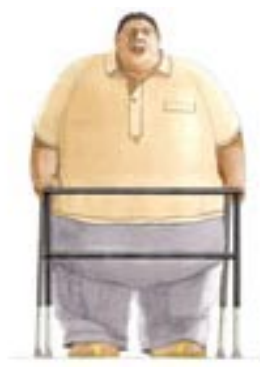

$\underline{\mathrm{B}(\mathrm{Bob})}$

- Uses walking frame or similar

- Can support himself to some degree

- Dependent on carer who is present in demanding situations

- Not physically demanding for carer

- Stimulation of remaining abilities (e.g. ambulation) is very important

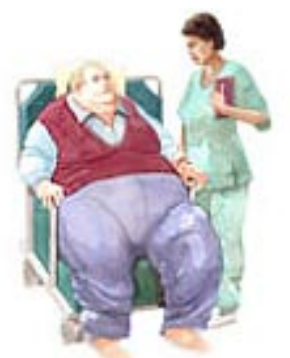

\section{$\underline{\text { C (Calvin) }}$}

- Sits in wheelchair

- Is able to partially bear weight on at least one leg 
- Has some trunk stability

- Dependent on carer in most situations

- Physically demanding for carer

- Stimulation of remaining abilities is very important

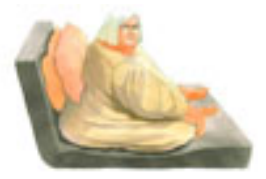

$\underline{\mathrm{D} \text { (Diana) }}$

- Sits in wheelchair

- No capacity to support herself at all

- Cannot stand unsupported and is not able to bear weight, not even partially

- Dependent on carer in most situations

- Physically demanding for carer

- Stimulation of remaining abilities is very important

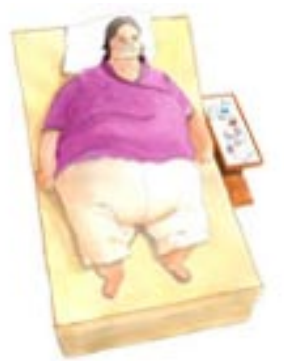

E (Ella)

- Passive resident

- Might be almost completely bedridden

- Often stiff, contracted joints

- Totally dependent

- Physically demanding for carer

- Stimulation and activation is not a primary goal 
Draft: 9/15/06

\section{Appendix 5 - Safe Patient Handling and Movement Algorithms}

[Nelson, 2005]

The plan and algorithms that follow can help assist developers to design appropriate patient handling technology for safe and effective patient care. It is provided here for the readers convenience. 


\section{Assessment Criteria and Care Plan for Safe Patient Handling and Movement}

I. Patient's Level of Assistance:

Independent - Patient performs task safely, with or without staff assistance, with or without assistive devices.

Partial Assist - Patient requires no more help than stand-by, cueing, or coaxing, or caregiver is required to lift no more than 35 lbs. of a patient's weight.

Dependent - Patient requires nurse to lift more than 35 lbs. of the patient's weight, or is unpredictable in the amount of assistance offered. In this case assistive devices should be used.

An assessment should be made prior to each task if the patient has varying level of ability to assist due to medical reasons, fatigue, medications, etc. When in doubt, assume the patient cannot assist with the transfer/repositioning.

II. Weight Bearing Capability

Full

Partial

None

\section{Bi-Lateral Upper Extremity Strength \\ Yes \\ No}

\section{Patient's level of cooperation and comprehension:}

Cooperative - may need prompting; able to follow simple commands.

Unpredictable or varies (patient whose behavior changes frequently should be considered as "unpredictable”), not cooperative, or unable to follow simple commands.

V. Weight: _ Height:

Body Mass Index (BMI) [needed if patient's weight is over 300]1:

If BMI exceeds 50, institute Bariatric Algorithms

The presence of the following conditions are likely to affect the transfer/repositioning process and should be considered when identifying equipment and technique needed to move the patient.

VI. Check applicable conditions likely to affect transfer/repositioning techniques.

Hip/Knee Replacements

History of Falls

Paralysis/Paresis

Unstable Spine

Severe Edema

Very Fragile Skin
Postural Hypotension

Severe Osteoporosis

Splints/Traction

Fractures

Respiratory/Cardiac Compromise Wounds Affecting Transfer/Positioning

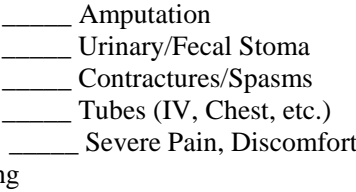

Amputation

Contractures/Spasm

Tubes (IV, Chest, etc.)

Comments:

\begin{tabular}{|c|c|c|c|}
\hline Algorithm & Task & $\begin{array}{c}\text { Equipment/ } \\
\text { Assistive Device }\end{array}$ & \# Staff \\
\hline 1 & Transfer To and From: Bed to Chair, Chair To Toilet, Chair to Chair, or Car to Chair. & & \\
\hline 2 & Lateral Transfer To and From: Bed to Stretcher, Trolley. & & \\
\hline 3 & Transfer To and From: Chair to Stretcher, or Chair to Exam Table. & & \\
\hline 4 & Reposition in Bed: Side-to-Side, Up in Bed. & & \\
\hline 5 & Reposition in Chair: Wheelchair and Dependency Chair. & & \\
\hline 6 & Transfer Patient Up from the Floor & & \\
\hline Bariatric 1 & Bariatric Transfer To and From: Bed to Chair, Chair to Toilet, or Chair to Chair & & \\
\hline Bariatric 2 & Bariatric Lateral Transfer To and From: Bed to Stretcher or Trolley & & \\
\hline Bariatric 3 & Bariatric Reposition in Bed: Side-to-Side, Up in Bed & & \\
\hline Bariatric 4 & Bariatric Reposition in Chair: Wheelchair, Chair or Dependency Chair & & \\
\hline Bariatric 5 & Patient Handling Tasks Requiring Access to Body Parts (Limb, Abdominal Mass, Gluteal Area) & & \\
\hline Bariatric 6 & Bariatric Transporting (Stretcher) & & \\
\hline Bariatric 7 & Bariatric Toileting Tasks & & \\
\hline
\end{tabular}

Sling Type (circle choice): Seated Seated (Amputation) Standing Supine Ambulation Limb Support

Sling Size:

Signature:

Date:

${ }^{1}$ If patient’s weight is over 300 pounds, the BMI is needed. For Online BMI table and calculator see: http://www.nhlbi.nih.gov/guidelines/obesity/bmi tbl.htm 
Draft: 9/15/06

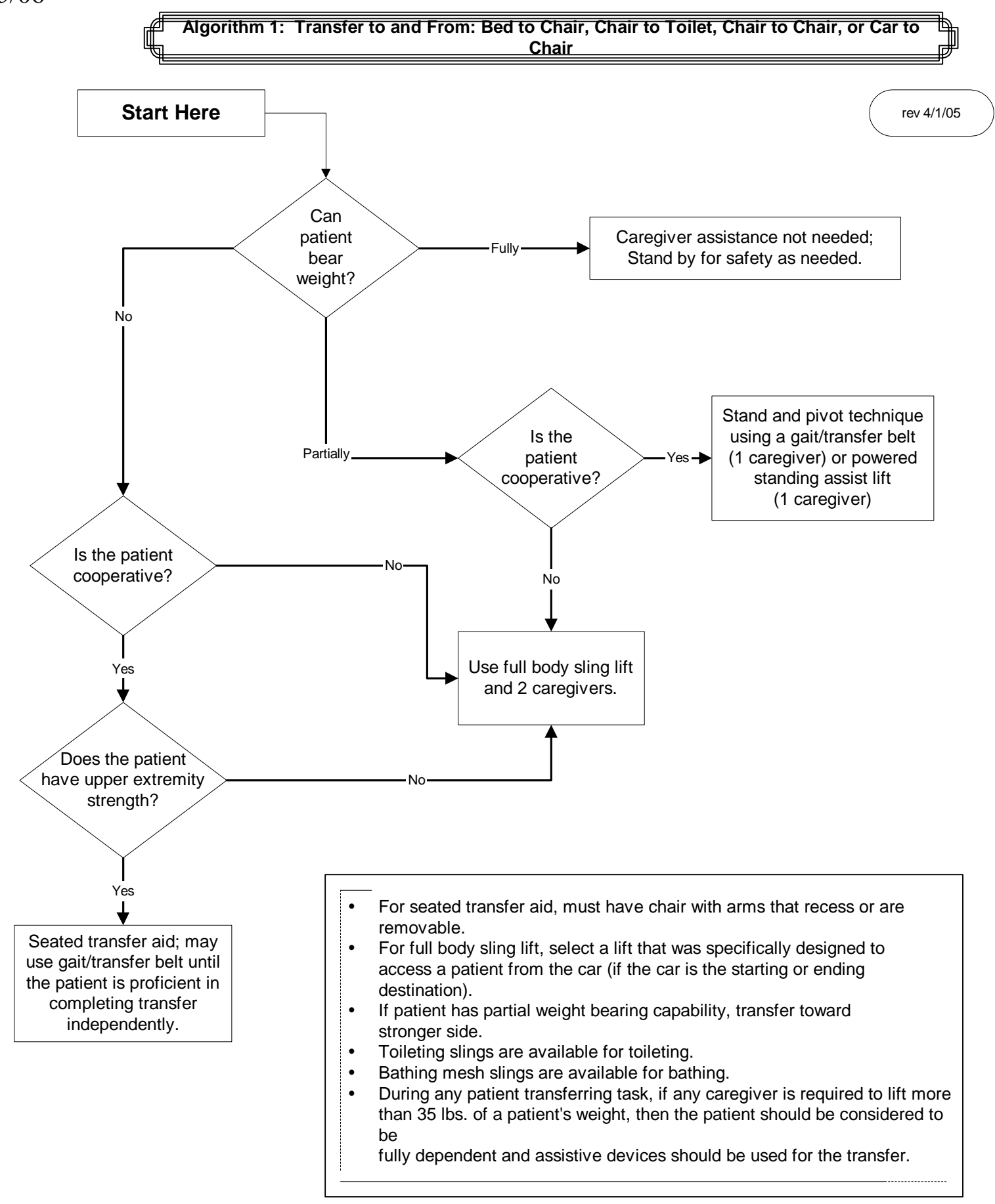


Draft: 9/15/06

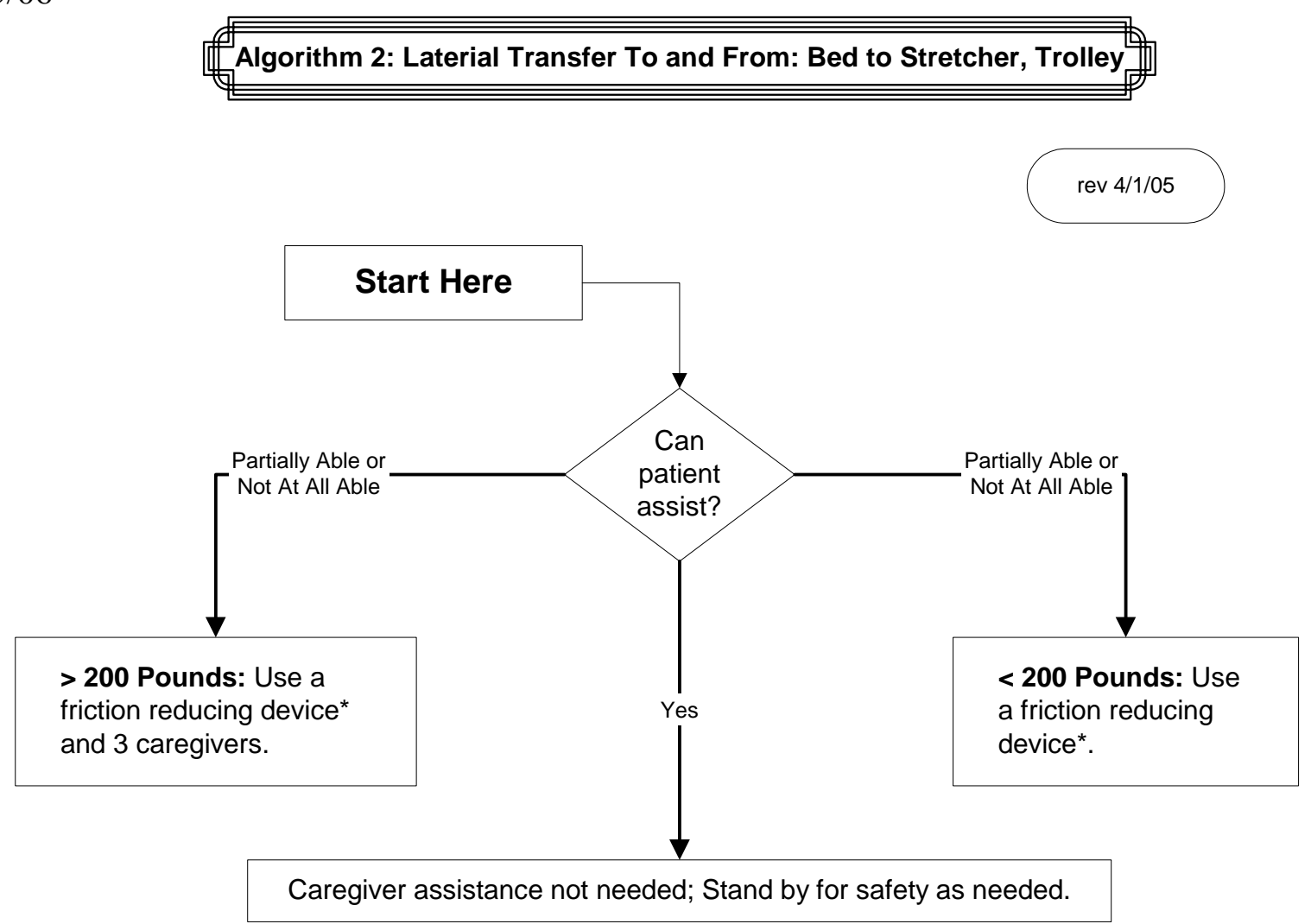

- Destination surface should be $1 / 2$ inch lower for all lateral patient moves.

- For patients with Stage III or IV pressure ulcers, care must be taken to avoid shearing force.

- During any patient transferring task, if any caregiver is required to lift more than $35 \mathrm{lbs}$. of a patient's weight, then the patient should be considered to be fully dependent and assistive devices should be used for the transfer. 
Draft: 9/15/06

Algorithm 3: Transfer To and From: Chair to Stretcher, or Chair to Exam Table

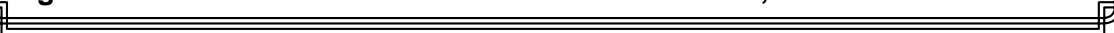

rev $4 / 1 / 05$

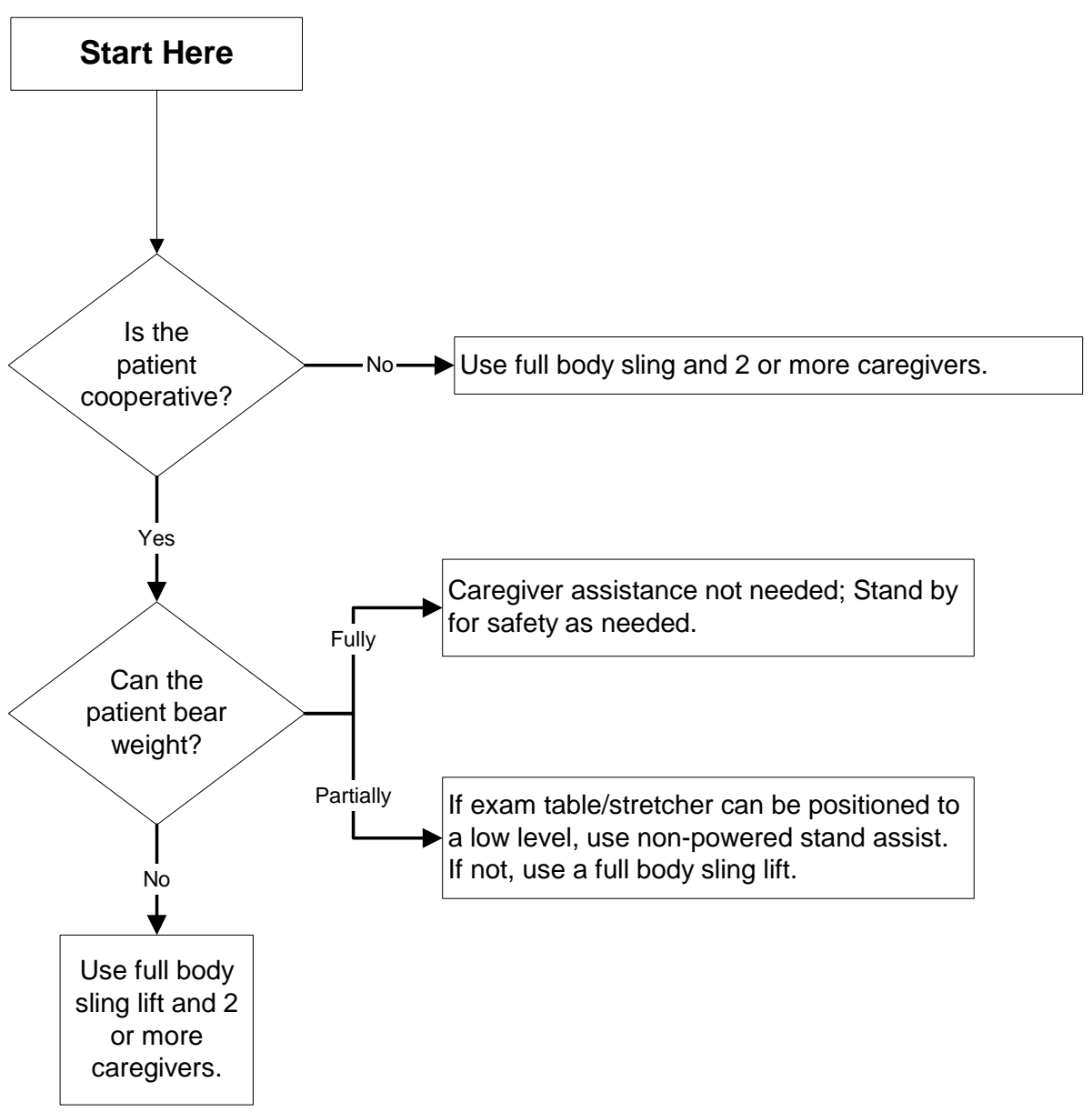

Comments:

- High/Low exam tables and stretches would be ideal.

- During any patient transferring task, if any caregiver is required to lift more than $35 \mathrm{lbs}$. of a patient's weight, then the patient should be considered to be fully dependent and assistive devices should be used for the transfer. 


\section{Algorithm 4: Reposition in Bed: Side-to-Side, Up in Bed}

rev $4 / 1 / 05$

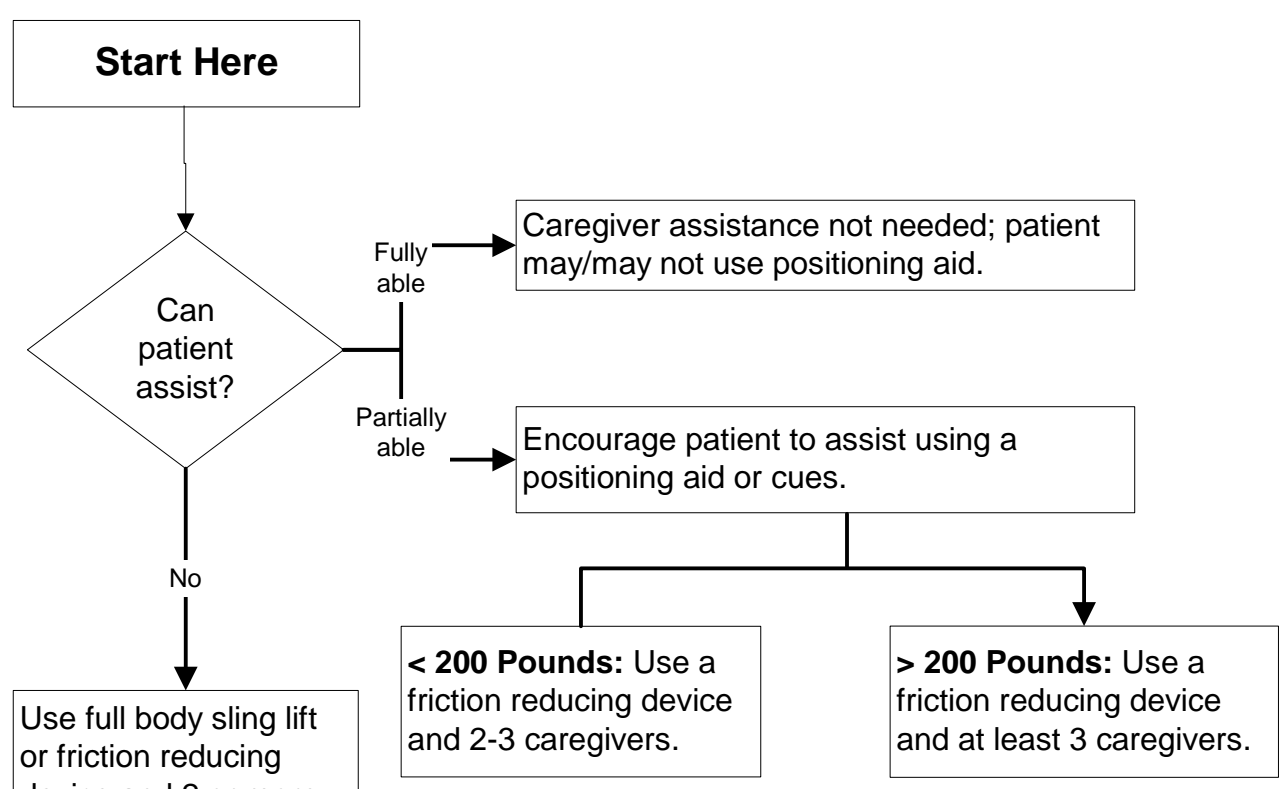
device and 2 or more caregivers.

- $\quad$ This is not a one person task: DO NOT PULL FROM HEAD OF BED.

- When pulling a patient up in bed, the bed should be flat or in a Trendelenburg position to aid in gravity, with the side rail down.

- For patients with State III or IV pressure ulcers, care should be taken to avoid shearing force.

- The height of the bed should be appropriate for staff safety (at the elbows).

- If the patient can assist when repositioning "up in bed," ask the patient to flex the knees and push on the count of three.

- During any patient transferring task, if any caregiver is required to lift more than $35 \mathrm{lbs}$ of a patients weight, then the patient should be considered to be fully dependent and assistive devices should be used for the transfer. 


\section{Algorithm 5: Reposition in Chair: Wheelchair and Dependency Chair}

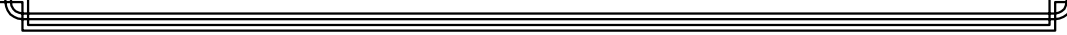

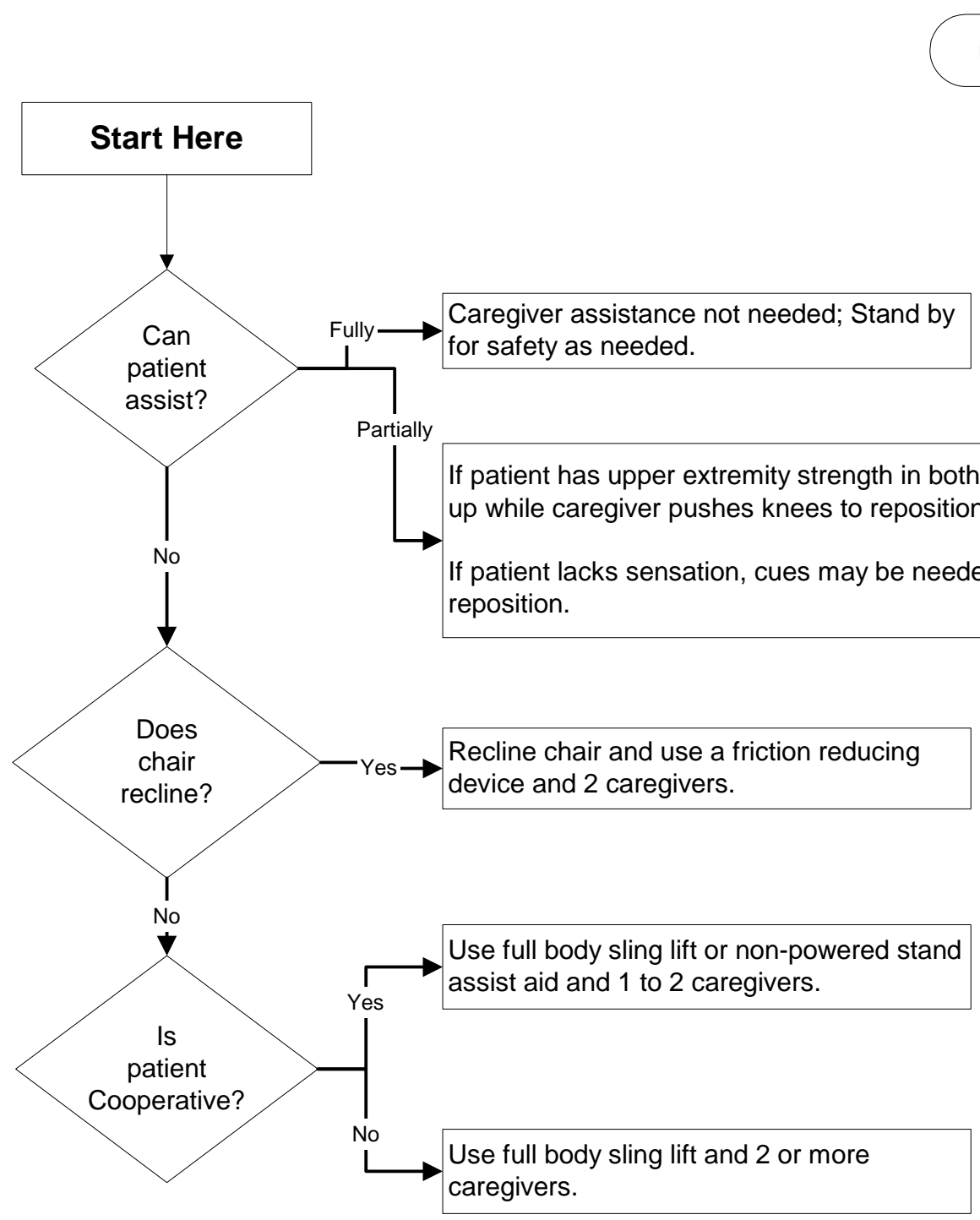

rev $4 / 1 / 05$

\section{Comments:}

- Take full advantage of chair functions, e.g., chair that reclines, or use or arm rest of chair to facilitate repositioning.

- Make sure the chair wheels are locked.

- During any patient transferring task, if any caregiver is required to lift more than $35 \mathrm{lbs}$. of a patient's weight, then the patient should be considered to be fully dependent and assistive devices should be used for the transfer. 
Draft 10/20/05

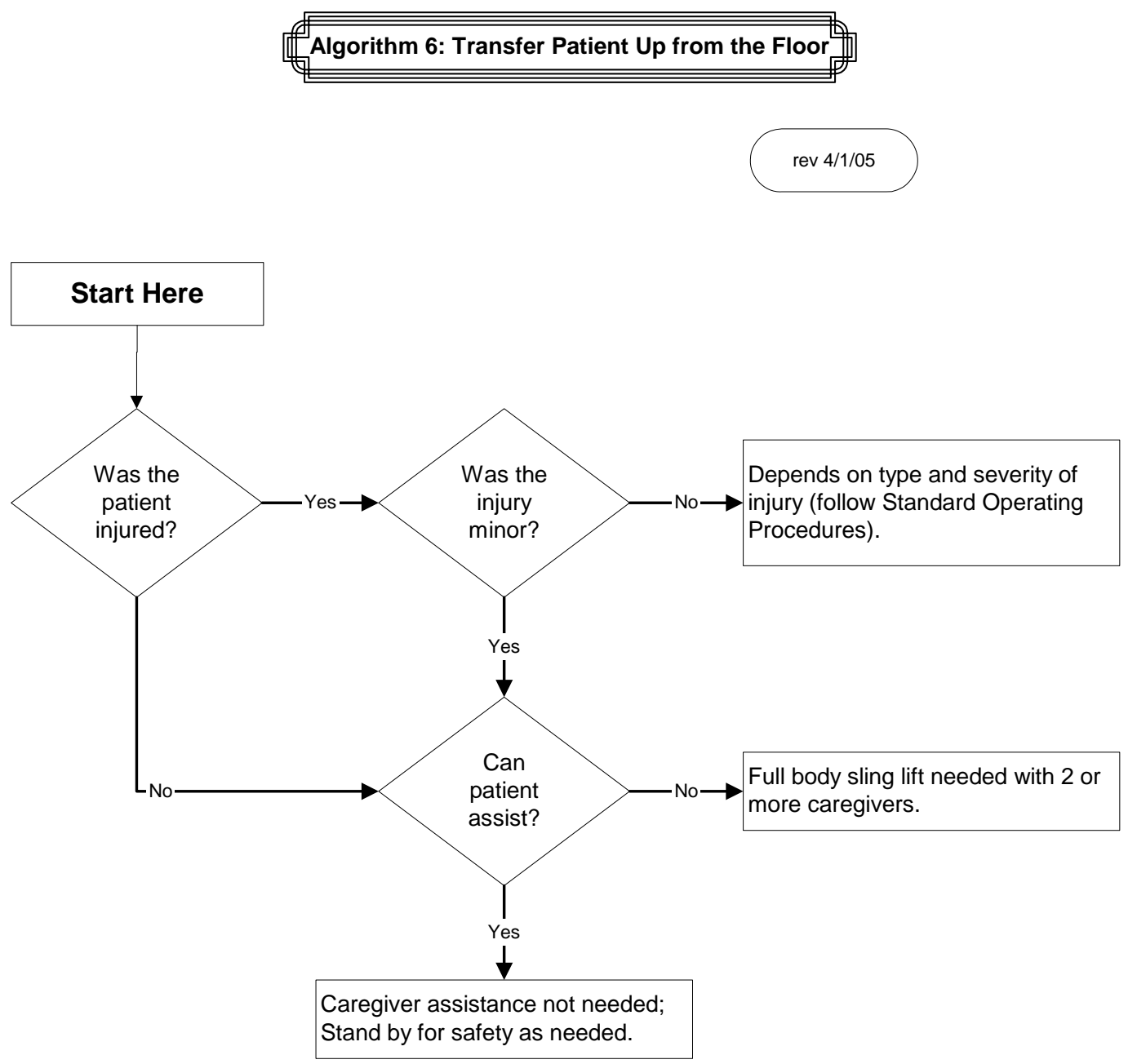

\section{Comments:}

- Use full body sling that goes all the way down to the floor (most of the newer models are capable of this).

- During any patient transferring task, if any caregiver is required to lift more than $35 \mathrm{lbs}$. of a patient's weight, then the patient should be considered to be fully dependent and assistive devices should be used for the transfer. 


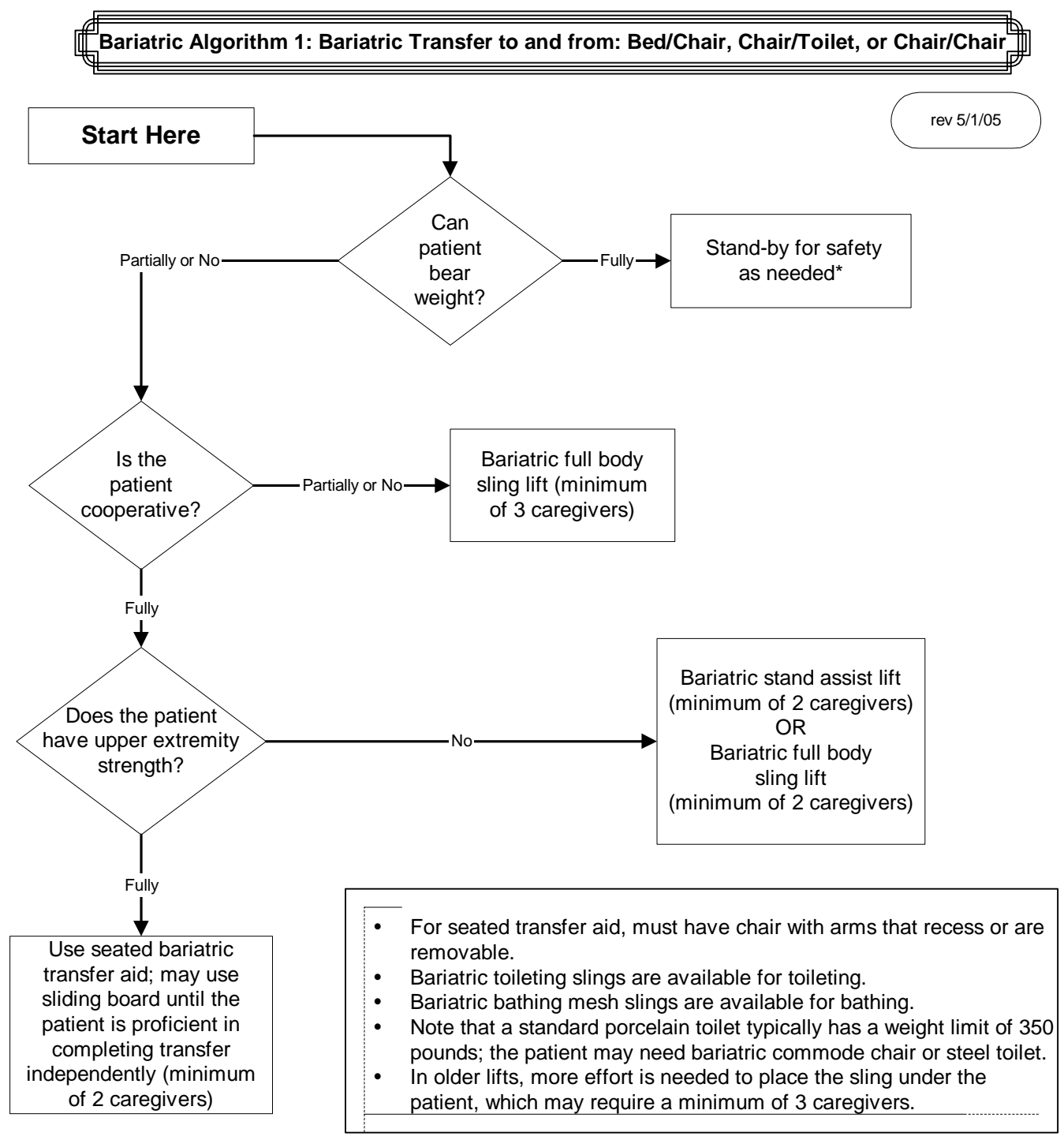

* "Stand-by for safety." In most cases, if a bariatric patient is about to fall, there is very little that the caregiver can do to prevent the fall. The caregiver should be prepared to move any items out of the way that could cause injury, try to protect the patient's head from striking any objects or the floor and seek assistance as needed once the person has fallen.

- If patient has partial weight-bearing capability, transfer toward stronger side.

- Consider using an abdominal binder if the patient's abdomen impairs a patient handling task.

- Assure equipment used meets weight requirements. Standard equipment is generally limited to 250-350 lbs. Facilities should apply a sticker to all bariatric equipment with "EC"(for expanded capabity) and a space for the manufacturer's rated weight capabity for that particular equipment model.

- Identify a leader when performing tasks with multiple caregivers. This will assure that the task is synchronized for increased safety of the healthcare provider and the patient.

- During any patient transferring task, if any caregiver is required to lift more than $35 \mathrm{lbs}$ of a patient's weight, then the patient should be considered to be fully dependent and assistive devices should be used for the transfer. 


\section{Bariatric Algorithm 2: Bariatric Lateral Transfer to and from: Bed/Stretcher, Trolley}
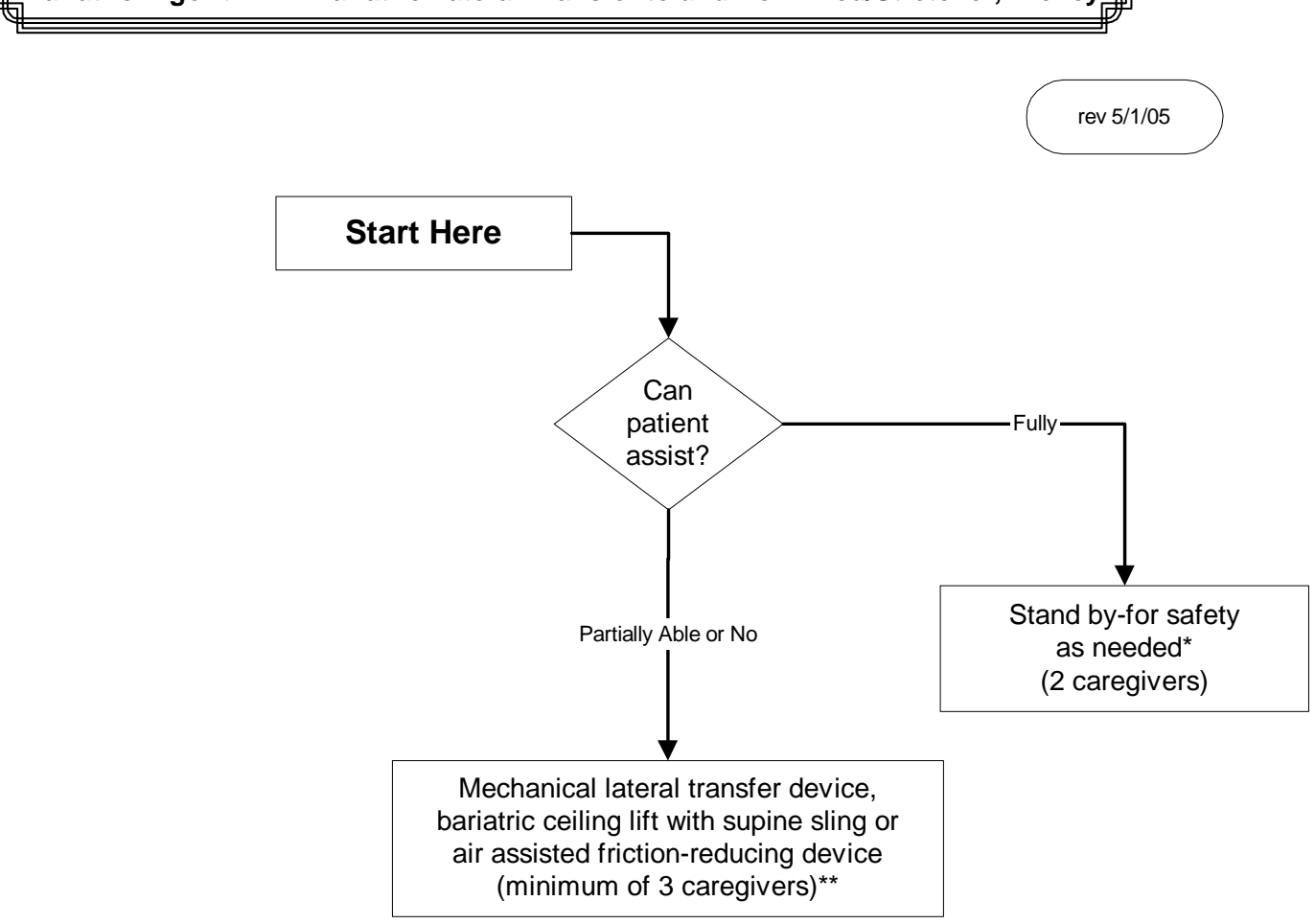

- The destination surface should be about $1 / 2$ " lower for all lateral patient moves.

- Avoid shearing force.

- Make sure bed is the right width, so excessive reaching by caregiver is not required.

- Lateral transfers should not be used with speciality beds that interfere with the transfer. In this case, use a bariatric ceiling lift with supline sling.

- Ensure bed or stretcher doesn't move with the weight of the patient transferring.

** Use a bariatric stretcher or trolley if patient exceeds weight capacity of traditional equipment.

* "Stand-by for safety." In most cases, if a bariatric patient is about to fall, there is very little that the caregiver can do to prevent the fall. The caregiver should be prepared to move any items out of the way that could cause injury, try to protect the patient's head from striking any objects or the floor and seek assistance as needed once the person has fallen.

- Assure equipment used meets weight requirements. Standard equipment is generally limited to 250-350 lbs. Facilities should apply a sticker to all bariatric equipment with "EC"(for expanded capabity) and a space for the manufacturer's rated weight capabity for that particular equipment model.

- If patient has partial weight-bearing capability, transfer toward stronger side.

- Consider using an abdominal binder if the patient's abdomen impairs a patient handling task.

- Identify a leader when performing tasks with multiple caregivers. This will assure that the task is synchronized for increased safety of the healthcare provider and the patient.

- During any patient transferring task, if any caregiver is required to lift more than $35 \mathrm{lbs}$ of a patients weight, then the patient should be considered to be fully dependent and assistive devices should be used for the transfer. 
Bariatric Algorithm 3: Bariatric Reposition in Bed: Side-to-Side, Up in Bed

\section{Start Here}

rev $5 / 1 / 05$

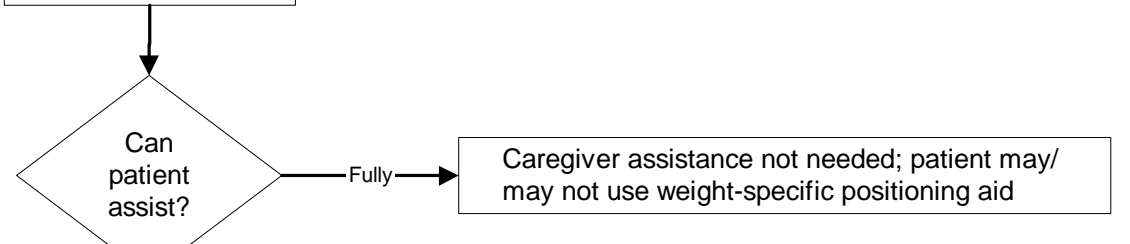

Y

Partially or No

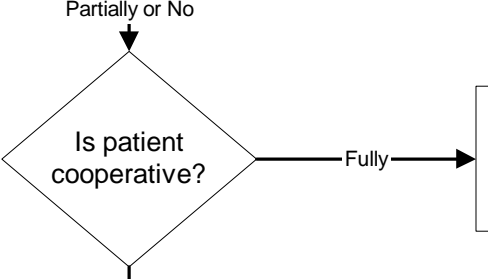

Bariatric ceiling lift with supine sling, air-assisted device or friction-reducing aid (minimum of 2 to 3 caregivers)

Bariatric ceiling lift with supline sling, air-assisted device or friction reducing aid (minimum of 3 caregivers)

- When pulling a patient up in bed, place the bed flat or in a Trendelenburg position (if tolerated and not medically contraindicated) to aid in gravity; the side rail should be down.

- Avoid shearing force.

- Adjust the height of the bed to elbow height.

- Mobilize the patient as early as possible to avoid weakness resulting from bed rest. This will promote patient independence and reduce the number of high risk tasks caregivers will provide.

- Consider leaving a friction-reducing device covered with drawsheet, under patient at all times to minimize risk to staff during transfers as long as it doesn't negate the pressure relief qualities of the mattress/overlay.

- Use a sealed, high-density, foam wedge to firmy reposition patient on side. Skid-resistant texture materials vary and come in set shapes and cut-your-own rolls. Examples include:

- $\quad$ Dycem (TM)

- Scoot-Guard (TM): antimicrobial; clean with soap and water, air dry.

- Posey-Grip (TM): Posey Grip does not hold when wet. Washable, reusable, air dry.

- If patient has partial weight-bearing capability, transfer toward stronger side.

- Consider using an abdominal binder if the patient's abdomen impairs a patient handling task.

- Assure equipment used meets weight requirements. Standard equipment is generally limited to 250-350 lbs. Facilities should apply a sticker to all bariatric equipment with "EC"(for expanded capabity) and a space for the manufacturer's rated weight capabity for that particular equipment model.

- Identify a leader when performing tasks with multiple caregivers. This will assure that the task is synchronized for increased safety of the healthcare provider and the patient.

- During any patient transferring task, if any caregiver is required to lift more than $35 \mathrm{lbs}$ of a patient's weight, then the patient should be considered to be fully dependent and assistive devices should be used for the transfer. 
Draft 10/20/05

Bariatric Algorithm 4: Bariatric Reposition in Chair: Wheelchair, Chair, or Dependency Chair

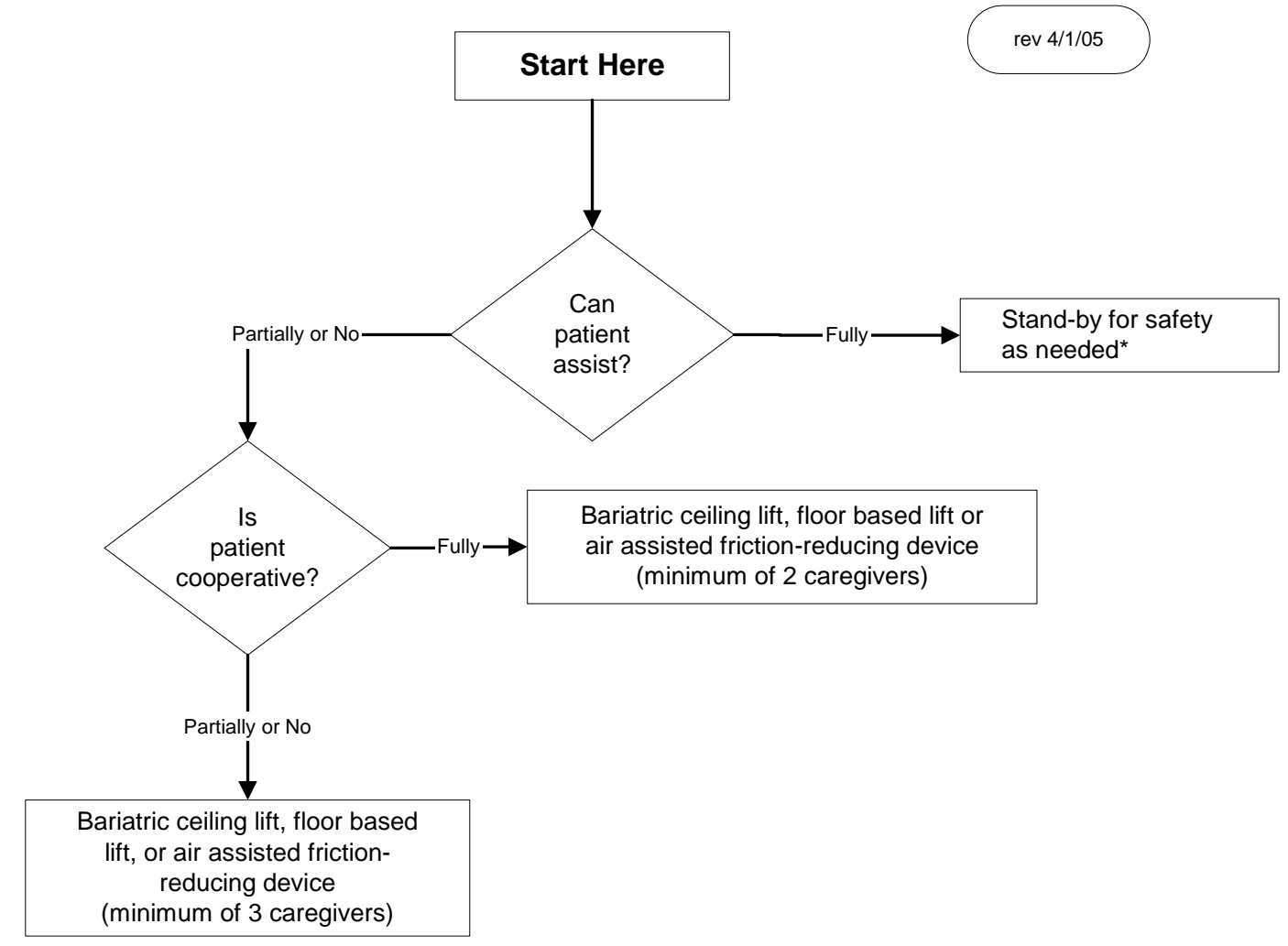

- Take full advantage of chair functions, e.g., chair that reclines, or use an arm rest of chair to facilitate repositioning.

- Make sure the chair wheels are locked

- Consider leaving the sling under the patient at all times to minimize risk to staff during transfers after carefully considering skin risk to patient and the risk of removing/replacing the sling for subsequent moves.

* "Stand-by for safety." In most cases, if a bariatric patient is about to fall, there is very little that the caregiver can do to prevent the fall. The caregiver should be prepared to move any items out of the way that could cause injury, try to protect the patient's head from striking any objects or the floor and seek assistance as needed once the person has fallen.

- If patient has partial weight-bearing capability, transfer toward stronger side.

- Consider using an abdominal binder if the patient's abdomen impairs a patient handling task.

- Assure equipment used meets weight requirements. Standard equipment is generally limited to 250-350 lbs. Facilities should apply a sticker to all bariatric equipment with "EC" (for expanded capabity) and a space for the manufacturer's rated weight capabity for that particular equipment model.

- Identify a leader when performing tasks with multiple caregivers. This will assure that the task is synchronized for increased safety of the healthcare provider and the patient.

- During any patient transferring task, if any caregiver is required to lift more than $35 \mathrm{lbs}$ of a patient's weight, then the patient should be considered to be fully dependent and assistive devices should be used for the transfer. 
Draft 10/20/05
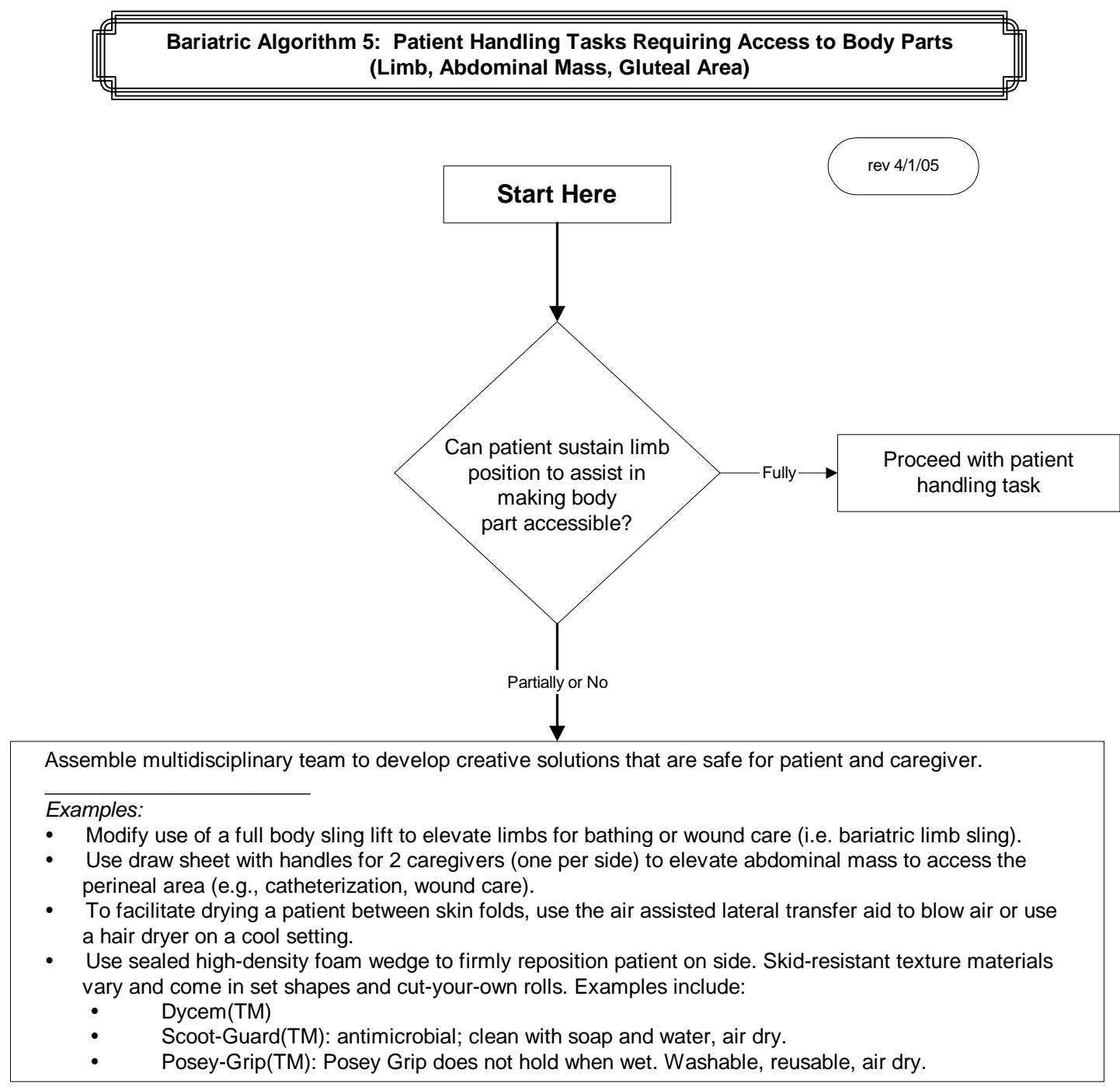

- A multidisciplinary team needs to problem solve these tasks, communicate to all caregivers, refine as needed and perform consistently.

- Consider using an abdominal binder if the patient's abdomen impairs a patient handling task.

- During any patient transferring task, if any caregiver is required to lift more than $35 \mathrm{lbs}$ of a patient's weight, then the patient should be considered to be fully dependent and assistive devices should be used for the transfer. 
Draft 10/20/05

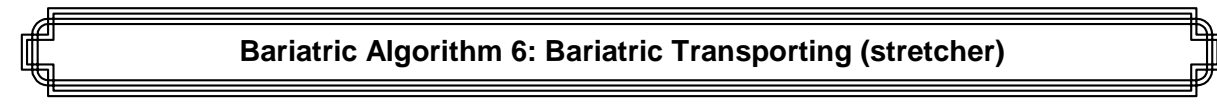

rev $5 / 1 / 05$

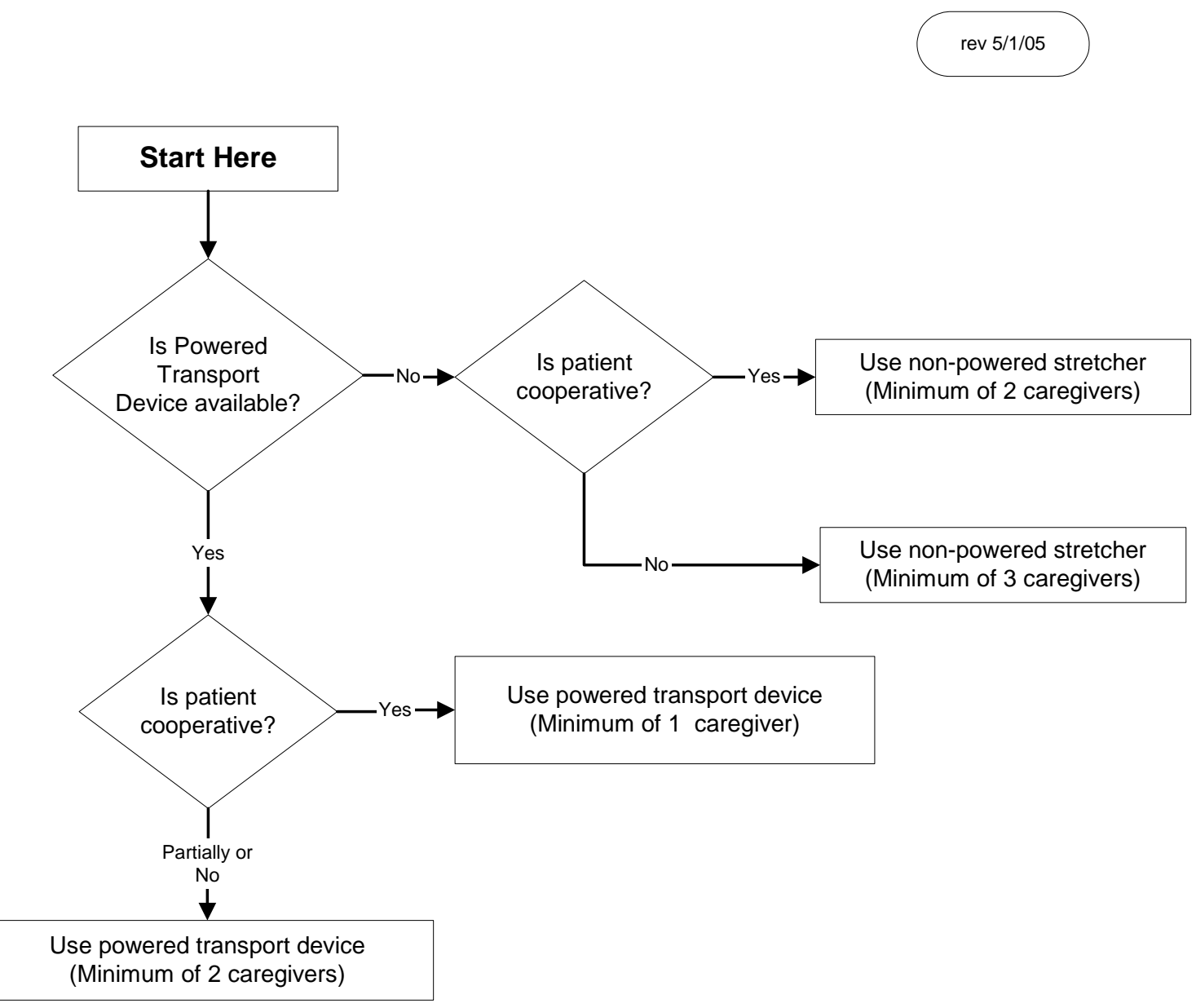

- If the patient has respiratory distress, the stretcher must have the capability of maintaining a high Fowler's position.

- Newer equipment often is easier to propel.

- If patient is uncooperative, secure patient in stretcher.

- During any patient transferring task, if any caregiver is required to lift more than $35 \mathrm{lbs}$ of a patient's weight, then the patient should be considered to be fully dependent and assistive devices should be used for the transfer. 
Draft 10/20/05

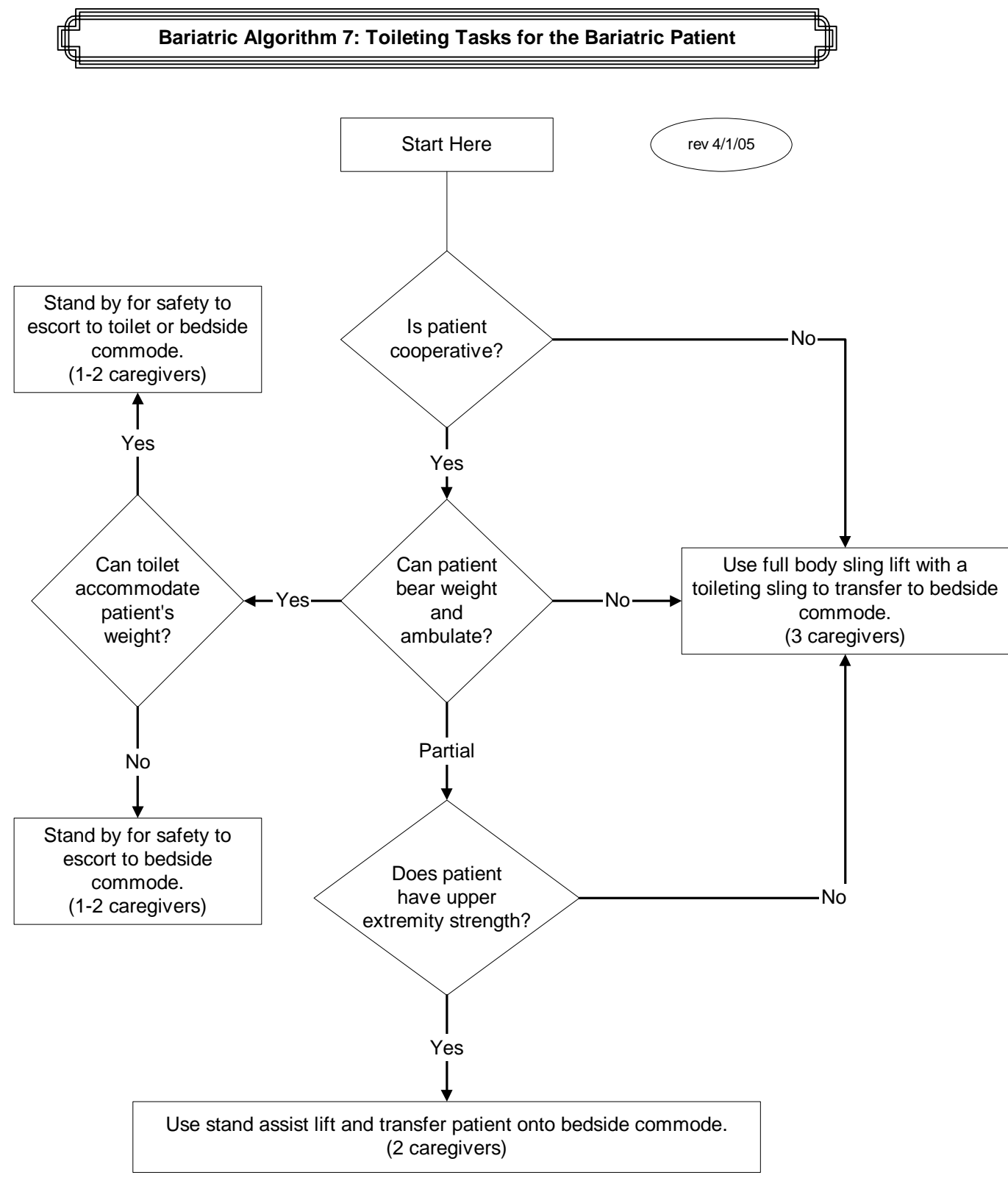

Considerations:

$>$ Is bathroom doorway wide enough to accommote entry of mechanical lift device and patient?

$>$ Assure equipment used meets weight requirements and is appropriately sized for patient.

> Typically, standard toilets are rated to $350 \mathrm{lbs}$. maximum capacity.

$>$ During any patient transferring task, if any caregiver is required to lift more than $35 \mathrm{lbs}$ of a patient's weight, then the patient should be considered to be fully dependent and assistive devices should be used for the transfer. 